Loma Linda University

TheScholarsRepository@LLU: Digital Archive of Research, Scholarship \& Creative Works

Loma Linda University Electronic Theses, Dissertations \& Projects

$6-2018$

\title{
Changes in the Morphology of Hypoglossal Motor Neurons in the Brainstem of Developing Rats
}

Paul Allen Williams

Follow this and additional works at: http://scholarsrepository.llu.edu/etd

Part of the Medical Physiology Commons

\section{Recommended Citation}

Williams, Paul Allen, "Changes in the Morphology of Hypoglossal Motor Neurons in the Brainstem of Developing Rats" (2018). Loma Linda University Electronic Theses, Dissertations \& Projects. 521.

http://scholarsrepository.1lu.edu/etd/521 Works. It has been accepted for inclusion in Loma Linda University Electronic Theses, Dissertations \& Projects by an authorized administrator of TheScholarsRepository@LLU: Digital Archive of Research, Scholarship \& Creative Works. For more information, please contact scholarsrepository@llu.edu. 


\title{
LOMA LINDA UNIVERSITY \\ School of Medicine \\ in conjunction with the \\ Faculty of Graduate Studies
}

\section{Changes in the Morphology of Hypoglossal Motor Neurons in the Brainstem of Developing Rats}

by

Paul Allen Williams

\begin{abstract}
A Dissertation submitted in partial satisfaction of the requirements for the degree

Doctor of Philosophy in Physiology
\end{abstract}

June 2018 
(C) 2018

Paul Allen Williams

All Rights Reserved 
Each person whose signature appears below certifies that this dissertation in his/her opinion is adequate, in scope and quality, as a dissertation for the degree Doctor of Philosophy.

Christopher G. Wilson, Associate Professor, Basic Sciences

, Chairperson

Denise L. Bellinger, Associate Research Professor, Pathology and Human Anatomy

John Buchholz, Professor, Basic Sciences

Kerby C. Oberg, Professor, Pathology and Human Anatomy

Sean M. Wilson, Associate Professor, Basic Sciences 


\section{ACKNOWLEDGEMENTS}

There are always many individuals who could be acknowledged, but there are those few who have made a notable contribution in that I would not be where I am without their time and efforts. Therefore, I would like to thank Mr. Joe Rondeau, my high school electronics shop teacher who encouraged me in science and technology leading to my interest in engineering. Thanks also to Dr. Roy Schubert, Dr. James Spaulding, Dr. Edward N. Roots, and Dr. Thomas Williams who taught, mentored, and befriended me during my undergraduate and graduate work at Louisiana Tech University. To Dr. Subrata Saha who accepted me into his laboratory and provided me with opportunities to expand my knowledge and skill set in my early years of Orthopaedic research. Finally, to Dr. Ian C. Clarke who guided, taught, and mentored me during my later years in Orthopaedic research.

I would also like to thank my committee members for their advice and direction. To Dr. Christopher G. Wilson, my PI, whose guidance, support, and feedback were crucial to my progress and development. Special thanks to Dr. Denise Bellinger who has been a source of incredible encouragement, assistance, and support as I have worked on the projects described in this dissertation.

To my family and friends, your love and support through this long endeavor has been invaluable. Thanks to my father and grandfathers for all the encouragement and mentorship they gave, each in their own way. And finally, I would like to thank God for providing me the undeserved opportunity to study His creation and marvel in its complexity and sophistication. 


\section{CONTENT}

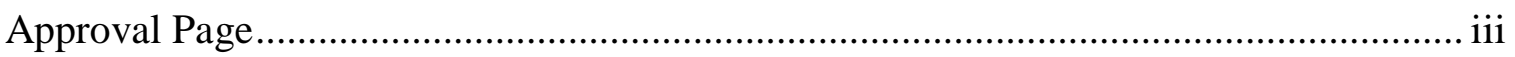

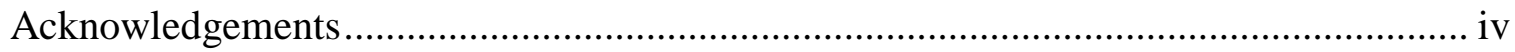

List of Figures ………………………........................................................... viii

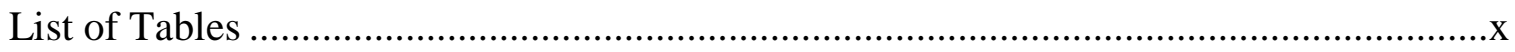

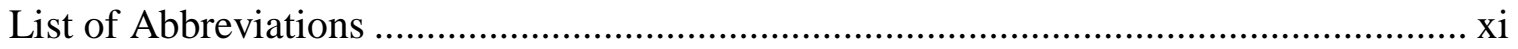

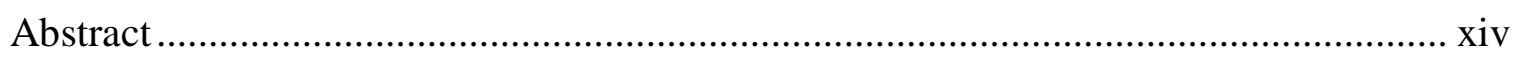

Chapter

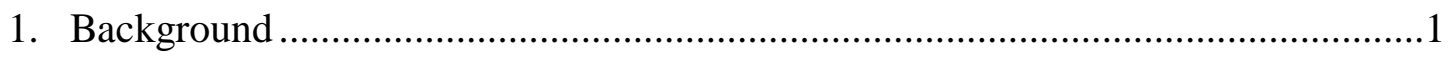

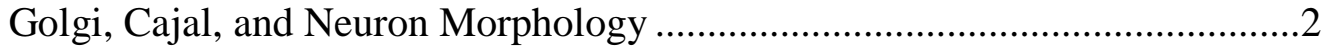

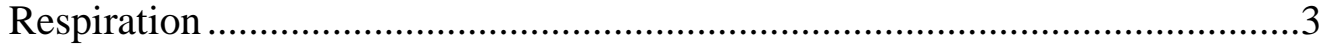

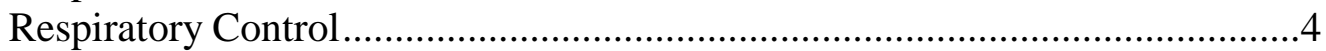

Hypoglossal Motoneurons ..........................................................................

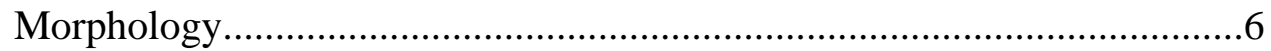

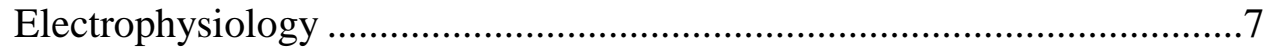

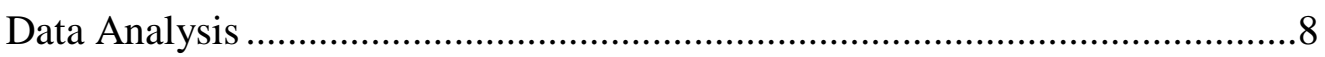

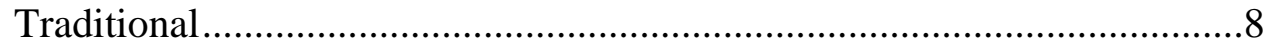

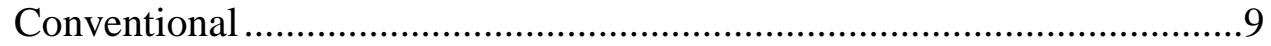

Distribution Analysis .............................................................................10

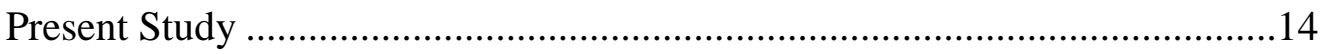

2. Changes in the morphology of hypoglossal motor neurons in the brainstem

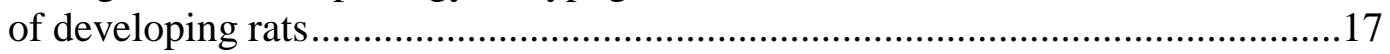

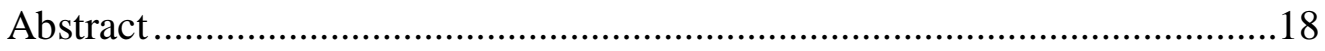

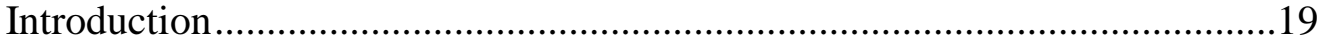

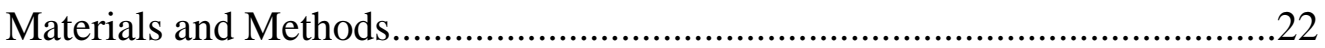

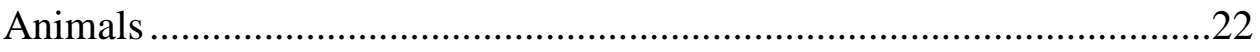

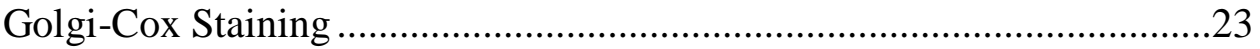

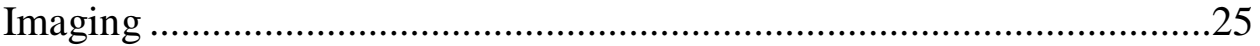

Morphological Measurements ................................................................2 
Location and Selection of Neurons............................................................30

Mapping of neuron locations ………………………........................30

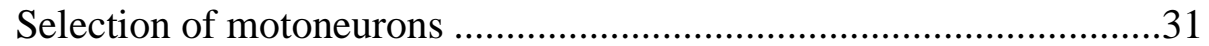

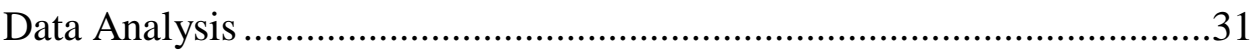

General analysis and comparisons ..................................................31

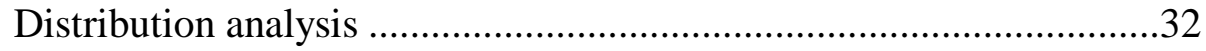

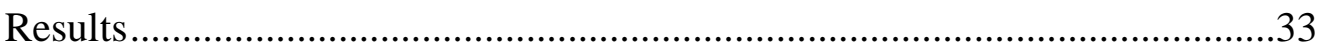

Soma Size, but Not Shape Changes with Age ............................................34

Soma Morphology is Unimodal, but not Necessarily Normally

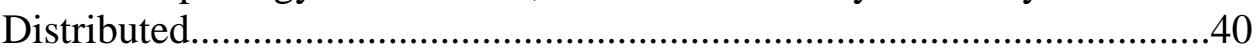

Extent and Complexity of the Dendritic Tree Increases with Age .............42

Dendrite Morphology: Normality and Unimodality ..................................55

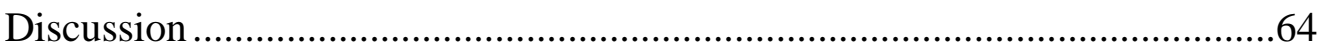

Postnatal Changes in Soma Morphology with Age …………...................66

Postnatal Changes in Dendrite Morphology ...............................................68

Hypoglossal Motoneuron Morphology: Normality, Unimodality,

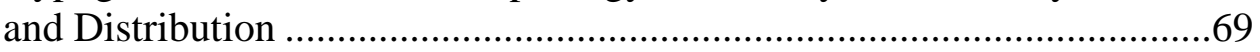

Comparison with Previous Studies .............................................................72

Impact of XII Morphology on Respiratory Function .................................74

Limitations and Future Directions ..........................................................75

Summary and Conclusions ...................................................................76

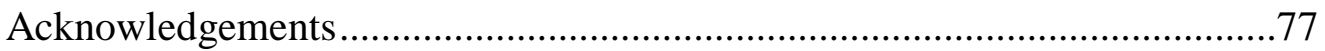

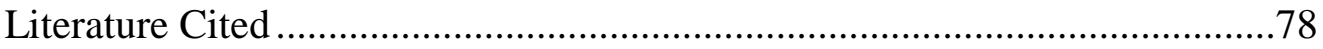

3. Modeling Hypoglossal Motoneurons in the Developing Rat ..................................84

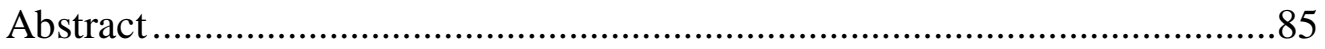

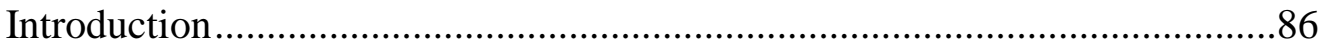

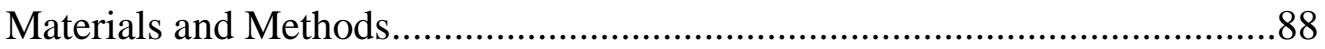

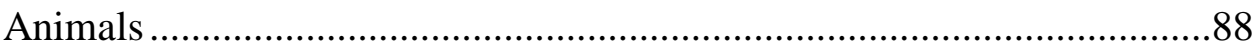

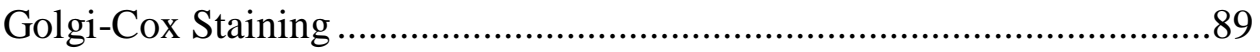

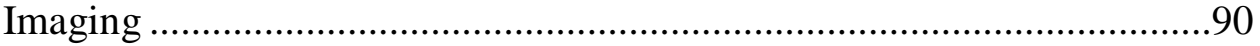

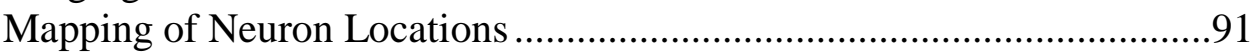

Selection of Motoneurons ...................................................................92

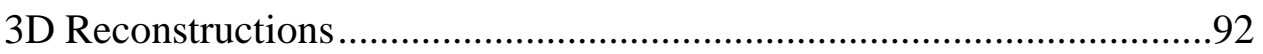

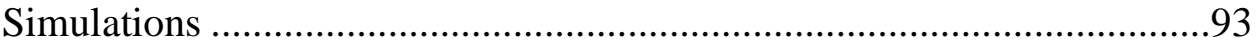

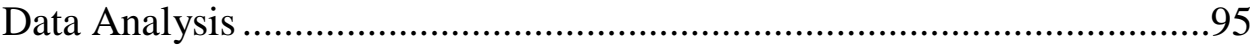


Results

3D Morphometrics and Electrophysiology .............................................96

Electrophysiology as a Function of Morphology...................................97

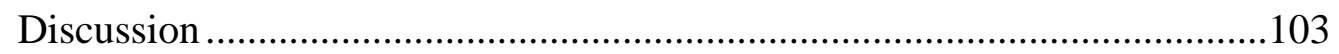

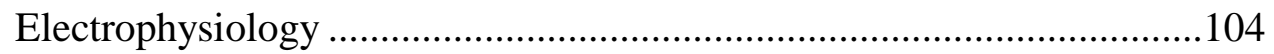

Electrophysiology as a Function of Morphology .................................105

Comparison with Previous Studies ....................................................106

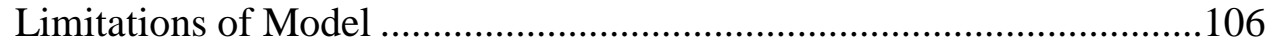

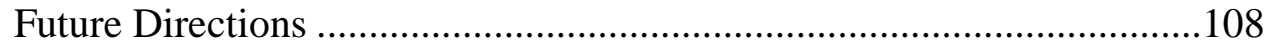

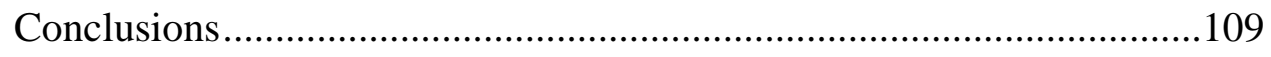

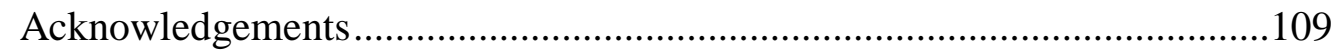

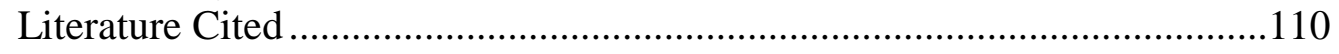

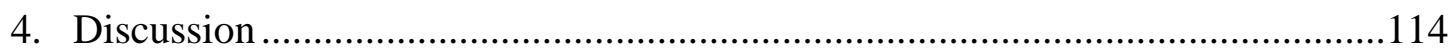

Developmental Morphology of Hypoglossal Motoneurons ..........................114

Developmental Electrophysiology of Hypoglossal Motoneurons .................116

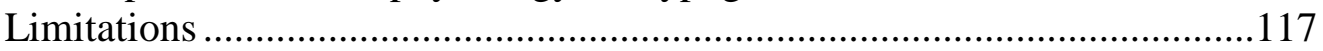

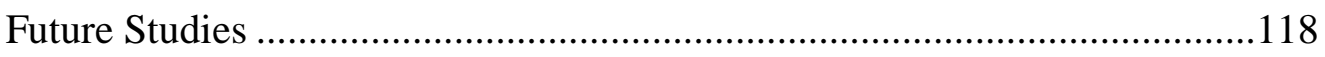

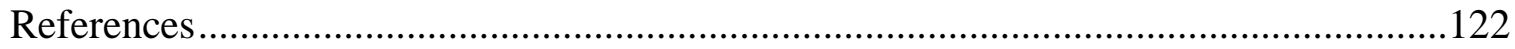




\section{FIGURES}

Figures $\quad$ Page

1. Graphs illustrating examples of different finite mixture models .........................13

2. Flowchart of the project showing morphology, electrophysiology, and

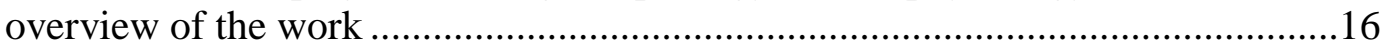

3. Morphology of hypoglossal motoneurons .........................................................28

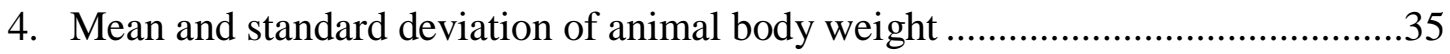

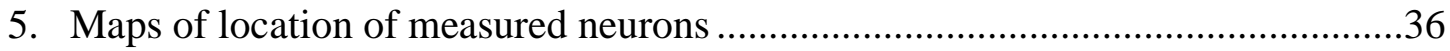

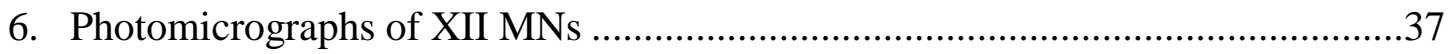

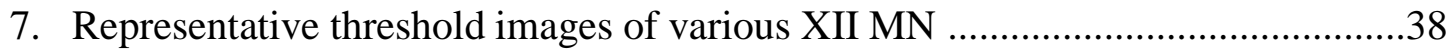

8. Cumulative distributions of the soma morphology..........................................39

9. Box plots of the neuron soma 2D morphometrics at each postnatal age ..............44

10. Pooled distributions for the ECD showing the finite mixture distribution

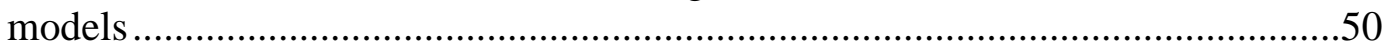

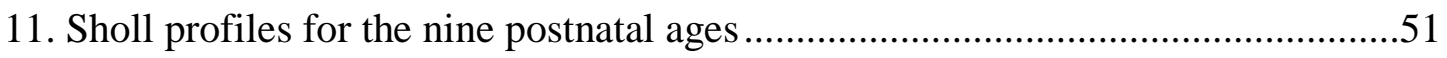

12. Representative threshold images of isolated XII MNs from P3, P10, and P21 with their respective Sholl profile and values.............................................52

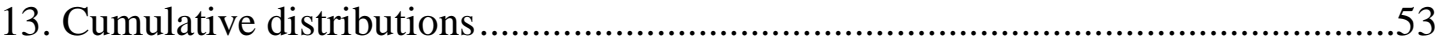

14. Box plots of the 2D Sholl analysis morphometrics measured at each of the

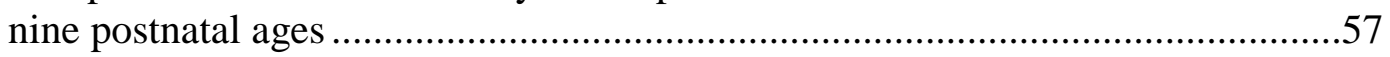

15. Distributions showing normal unimodal, non-normal unimodal, and nonnormal non-unimodal data

16. Pooled distributions for the maximum intersection radius showing the finite mixture distribution models

17. Threshold images of $\mathrm{P} 1, \mathrm{P} 10$, and $\mathrm{P} 12$ sections which have two isolated hypoglossal neurons with different morphologies

18. Morphology of hypoglossal motoneurons at P3, P10, and P17. The 3D reconstructions and their associated Sholl profiles are shown 


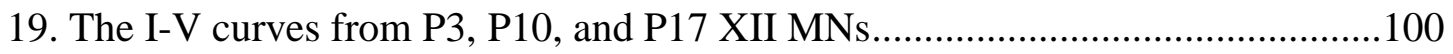

20. Action potentials and mAHP from P3, P10, and P17 XII MNs .........................101

21. Regression analysis of passive and active biophysical properties of XII MNs as a function of morphology ........................................................... 102 


\section{TABLES}

Tables

1. Number of animals available for measurement per litter for the nine

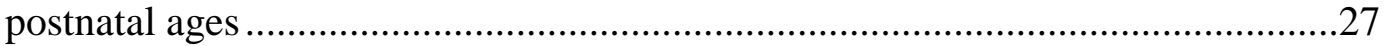

2. Number of XII MN's analyzed (per litter) for the nine postnatal ages.................29

3. Shapiro-Wilk normality test results for soma morphology..................................45

4. Soma morphology summary statistics (mean \pm std) for the three age groups .......46

5. Results of fitting two normal distributions to various parameters for P1-P5 (P1, P3, \& P5)

6. Results of fitting two normal distributions to various parameters for P7-P12 (P7, P10, \& P12)

7. Results of fitting two normal distributions to various parameters for P13-P21 (P13, P17, \& P21)

8. Shapiro-Wilk normality test results for dendrite morphology

9. Results from Hartigans' dip test for unimodality/multimodality for dendrite morphology

10. Dendrite morphology summary statistics (mean \pm std) for the three age groups

11. Summary statistics of morphological and biophysical properties from XII MNs. 


\section{ABBREVIATIONS}

\begin{tabular}{ll} 
CNS & Central nervous system \\
XII & Hypoglossal nucleus \\
MN & Motoneuron or motor neuron \\
XII MN & Hypoglossal motoneuron \\
P & Postnatal day \\
2D & Two dimensional \\
3D & Three dimensional \\
SIDS & Sudden infant death syndrom \\
nTS & Nucleus tractus solitari \\
pBC & Pre-Bötzinger complex \\
GABA & Gamma-aminobutyric acid \\
Q-Q plots & Quantile-Quantile plots \\
ANOVA & Analysis of variance \\
E & Embryonic day \\
ECD & Equivalent circular diameter \\
AR & Aspect Ratio \\
FF & Form Factor \\
PDF & Probability density function \\
CDF & Not applicable \\
Avg & Number \\
\hline &
\end{tabular}




\begin{tabular}{|c|c|}
\hline Std & Standard deviation \\
\hline $\mathrm{CV}$ & Coefficient of variation \\
\hline$\mu \mathrm{m}$ & Micrometer \\
\hline MajDia & Major diameter \\
\hline $\mathrm{V}$ & Membrane potential \\
\hline $\mathrm{t}$ & Time \\
\hline $\mathrm{C}_{\mathrm{m}}$ & Membrane capacitance \\
\hline $\mathrm{I}_{\mathrm{x}}$ & Ionic current for any ion $\mathrm{x}$ \\
\hline$\overline{\mathrm{g}}_{\mathrm{x}}$ & maximum conductance for any ion $\mathrm{x}$ \\
\hline $\mathrm{y}_{\mathrm{x}}$ & Product of activation or inactivation gating variables \\
\hline$E_{x}$ & Equilibrium potential for any ion $\mathrm{x}$ \\
\hline $\mathrm{I}_{\mathrm{Na}}$ & Fast sodium channel current \\
\hline $\mathrm{I}_{\mathrm{NaP}}$ & Persistent sodium channel current \\
\hline $\mathrm{I}_{\mathrm{k}}$ & Potassium channel current \\
\hline $\mathrm{I}_{\mathrm{A}}$ & Fast-transient potassium current \\
\hline Ileak & Leakage current \\
\hline$I_{\text {pas }}$ & Passive ionic current \\
\hline $\mathrm{I}_{\mathrm{h}}$ & Hyperpolarization-activated current \\
\hline $\mathrm{g}_{\mathrm{Na}}$ & Fast sodium conductance \\
\hline $\mathrm{g}_{\mathrm{K}}$ & Potassium conductance \\
\hline gleak & Leakage conductance \\
\hline gas & Passive conductance \\
\hline $\mathrm{g}_{\mathrm{NaP}}$ & Persistent sodium conductance \\
\hline
\end{tabular}




$\begin{array}{ll}g_{A} & \text { Fast-transient potassium conductance } \\ g_{h} & \text { Hyperpolarization-activated conductance } \\ \mathrm{E}_{\mathrm{Na}} & \text { Fast sodium membrane potential } \\ \mathrm{E}_{\mathrm{K}} & \text { Potassium membrane potential } \\ \mathrm{E}_{\text {leak }} & \text { Leakage membrane potential } \\ \mathrm{E}_{\mathrm{pas}} & \text { Passive membrane potential } \\ \mathrm{E}_{\mathrm{h}} & \text { Hyperpolarization-activated membrane potential } \\ \mathrm{mV} & \text { Millivolts } \\ \mathrm{nA} & \text { Nanoamperes } \\ \mathrm{ms} & \text { Milliseconds } \\ \mathrm{R}_{\mathrm{peak}} & \text { Peak resistance } \\ \mathrm{mAHP} & \text { Medium Afterhyperpolarizaton } \\ \mathrm{SK} & \text { Small-conductance calcium-activated potassium channel } \\ \mathrm{Na} & \text { Voltage-gated sodium channel }\end{array}$




\title{
ABSTRACT OF THE DISSERTATION OR THESIS
}

\section{Changes in the Morphology of Hypoglossal Motor Neurons in the} Brainstem of Developing Rats

by

\author{
Paul Allen Williams \\ Doctor of Philosophy, Graduate Program in Physiology \\ Loma Linda University, June 2018 \\ Christopher G. Wilson, Chairperson
}

The autonomic brainstem generates and modifies breathing rhythm by integrating inputs from chemo- and mechanosensors in the viscera while coordinating descending outputs from higher CNS structures. Hypoglossal motoneurons (XII MNs) receive inputs from respiratory premotor neurons in the medulla. Previous studies in rodents have demonstrated significant changes in breathing control during the first three weeks of life, with a sensitive period at 10 to 13 days post-birth (P10-P13) characterized by pronounced changes in neurotransmitters, receptors, excitation-inhibition balance, and breathing. However, age-dependent morphological changes of XII MNs during the first three weeks post-birth and especially during this sensitive period, have not been thoroughly studied. In this study, we comprehensively characterized and quantified the postnatal morphological changes in rat XII MNs. We hypothesized that morphological changes occur in XII MN morphology and arbor complexity corresponding to the functionally-defined sensitive period observed at P10-P13. To test this hypothesis, we used innovative statistical approaches to quantify and compare developmental changes in Golgi-Cox stained XII MNs at nine postnatal ages between P1-P21. Additionally, we performed 3D reconstructions of the neurons importing these geometries into the 
modeling environment NEURON to simulate the biophysical properties of XII MNs. Soma size increased $\sim 40 \%$ from P1 to P21, with no significant change in shape. However, dendritic arborization increased in extent and complexity with branching of neurons significantly increasing from P1 through P13, with the greatest increase at P10P13 based on the Sholl method. Three age groups 1) P1-P5, 2) P7-P12, and 3) P13-P21 were found as possible windows of development. We also found that at specific ages certain parameters such as soma size and dendritic complexity were non-normally distributed. I found support for differences in the density of selected voltage-gated ion channels with age and correlations between passive electrophysiological properties and morphology. Although a direct relationship was not found between morphology and the active properties, I did find support for an indirect relationship. Our detailed characterization of XII MN morphological development establishes a foundation for the study and elucidation of morphological changes caused by maternal and perinatal conditions using a rigorous approach. 


\section{CHAPTER ONE}

\section{BACKGROUND}

At birth, autonomic regions in the brainstem, which generate and control respiratory rhythm, which is critical throughout life, are not fully developed (Feldman et al., 2013; Feldman and Del Negro, 2006; Feldman and Smith, 1995). Normal control of breathing develops in the first few weeks after birth with motoneurons (MNs) of the hypoglossal nucleus (XII) and other brainstem nuclei providing the drive to the tongue and upper airways and are used in in vitro models to provide an index of fictive inspiratory effort (Feldman et al., 2013; Feldman and Del Negro, 2006). The coordination of the tongue and upper airway between speech and breathing is also an important aspect that motivates the study of XII MN development. Because $80 \%$ or more of the neurons in the XII are MNs that are readily distinguishable from the interneurons and play an active role in inspiration during development, they are a convenient and accessible population of neurons for morphological and electrophysiological study (Cooper, 1981; McClung and Goldberg, 1999). Although the effects of various factors such as nicotine and inflammation on respiratory development have been studied (Fregosi and Pilarski, 2008; Powell et al., 2016; Rourke et al., 2016), very little is known about these conditions on morphological development. One possible reason for this is that changes in the normal maturation of breathing patterns has been studied (Liu et al., 2009, 2006), but little is known about the normal maturation of XII MN morphology.

Therefore, in this work my objective was to comprehensively characterize and quantify the normal maturation of somatic and dendritic tree morphology as has been done for breathing pattern. In addition, I simulated the electrophysiological behavior of 
XII MNs based on measured 3D geometries comparing these results to results from electrophysiological experiments. I employed Golgi-Cox staining, 2D Sholl analysis, 3D reconstructions, and the NEURON simulation environment to measure morphology and simulate electrophysiology.

\section{Golgi, Cajal and Neuron Morphology}

In the late 1800 's into the early 1900 's two main theories of the nervous system were debated. On the one hand some such as Golgi believed that the nervous was comprised of a diffused network formed by anastomosis of axonal processes (reticular doctrine). Others such as Cajal believed that the nervous system was composed of individual nerve cells that were connected (neuron doctrine) (De Carlos and Borrell, 2007). Although Cajal and Golgi defended different concepts of the nervous system organization, Cajal's work was based on the utilization of modified versions of Golgi's histological techniques for staining neurons first published in 1873 (De Carlos and Borrell, 2007). Since then the Golgi staining technique has been utilized, improved, adapted to other application, and still in some respects the Gold Standard in studying neuron morphology (Koyama, 2013). The original Golgi stain and the variety of modified techniques such as Golgi-Cox and Rapid Golgi has been used in bright-field microscopy, electron microscopy, and to differentially stain glia cells (Koyama, 2013; Koyama et al., 2013; Levine et al., 2013; Martinez-Tellez et al., 2005; Ranjan and Mallick, 2012). Some of the advantages of Golgi staining is the resolution obtained while dendrites along with dendritic spines are identified with good detail. Despite the advantageous of modern histological and staining methods, the utility and benefits that the variety of Golgi stains 
have allows it to still be a commonly employed technique in the study of neuron morphology.

\section{Respiration}

Respiration is a fundamental autonomic function that involves the coordination of the diaphragm, lung volume, airway tone, and control of the tongue to be maintained continuously over the duration of an organism's life. Even though the control of breathing is fundamental to all mammals, our knowledge of the underlying neural circuitry that generates breathing rhythm is still nascent (Feldman et al., 2013; Feldman and Del Negro, 2006). Whether an individual breaths through the nose or mouth and its impact on health has been the subject of study (Fujimoto et al., 2009; Leal et al., 2016; Macari et al., 2012; Padzys et al., 2011). However, developmental changes in the breathing mode (nasal versus mouth) has received very little, if any study (Hall, 2005; Mortola and Fisher, 1981). Although breathing mode is clinically important, details on shifts between obligate mouth to nasal breathing during postnatal development are lacking. The neural network that generates and transmits breathing motor pattern is located in the brainstem and must be functional prior to birth (approximately embryonic day 15 in rats and mice (Thoby-Brisson and Ramirez, 2001, 2000) and continues to develop and remodel in the first weeks of life (Harper and Kinney, 2010; Kinney, 2009; Wong-Riley and Liu, 2005). If the breathing neural network fails or is unable to generate a physiologically appropriate rhythm, pathological breathing patterns can result, and these can impair the animal' s well-being. During early life, breathing disorders such as apnea of prematurity, periodic breathing, and Sudden Infant Death Syndrome (SIDS) 
(Erickson et al., 2007; Kinney, 2009; Martin and Wilson, 2009). Historical work in breathing control has provided an understanding of how metabolic rate affects breathing across the stages of development—-from birth to adulthood (Liu et al., 2006; Mortola, 1984), but we do not yet understand how breathing regulation and control are correlated with anatomical structure and synaptic remodeling of neurons embedded within the breathing circuit (Feldman et al., 2013; Feldman and Del Negro, 2006; Wong-Riley et al., 2013). Understanding how the breathing neural network is remodeled during early life is key to a deeper understanding of the role that morphology plays in reliable transmission of breathing rhythm in the perinatal period.

\section{Respiratory Control}

Regions of the brainstem such as the nucleus tractus solitarii (nTS), PreBötzinger Complex (pBC), and hypoglossal nucleus (XII) are key to the generation and transmission of the breathing pattern (Lindsey et al., 2012; Wong-Riley and Liu, 2005), however we understand very little about the structural changes across the early developmental window of autonomic circuitry. While it has been known for some time that neural network behavior is influenced by the morphology of neurons and connectivity is altered due to changes in neurotransmitter release and receptor up- and down-regulation (Ascoli et al., 2009; Donohue and Ascoli, 2008; Krichmar et al., 2002). Yet, even fundamental knowledge about these changes in the brainstem are limited (Fregosi, 2011; R. Kanjhan et al., 2016). The motoneurons of the hypoglossal nucleus (XII) provide the drive to the tongue and upper airways and are used in in vitro models to provide an index of fictive inspiratory effort (Koizumi et al., 2008; Smith et al., 1991). It 
has previously shown that there are age-dependent changes in hypoglossal motoneuron output drive (Fietkiewicz et al., 2011).

Detailed studies of the development of respiration and respiratory control have been conducted in only the past couple of decades. During the first three weeks post-birth (P0-P21) cytochrome oxidase generally increases in the pBC, nTS, XII, and other nuclei of the brain stem (Liu and Wong-Riley, 2003, 2001). However, at P3 and P4 there is plateau in the level of cytochrome oxidase that occurs at P3 and P4 in the pBC, while there is a prominent decrease at $\mathrm{P} 12$. These changes at $\mathrm{P} 3, \mathrm{P} 4$, and $\mathrm{P} 12$ in the $\mathrm{pBC}$ also exhibit increases of inhibitory drive mediated by GABA and glycine and decreased in excitatory drive mediated by glutamate activity (Liu et al., 2006; Wong-Riley and Liu, 2005). In brainstem nuclei such as the XII, nTS, and pBC there is mostly a steady increase in cytochrome oxidase up to P7 (Liu and Wong-Riley, 2003). During the second week of postnatal life (P8-P14), there are shifts in excitation-inhibition balance (both neurotransmitters and receptors) and cytochrome oxidase levels, especially on days 10 , 11, 12, and 13 in most brainstem nuclei (Liu et al., 2006; Liu and Wong-Riley, 2003).

Ventilatory changes are also seen from P0 to P21 with an increase in breathing frequency during the hypoxic ventilatory response at P0-P6 (Liu et al., 2009, 2006). At P10-P21 there is a decrease in breathing frequency, but an increase in tidal volume while there is a decrease in minute volume at P10-P13 with an increase at P14-P21 (Liu et al., 2009, 2006). Postnatal days P10-P13 have been referred to as a "sensitive period" in which respiratory development exhibits pronounced changes in frequency and variability of breathing pattern. This "sensitive period" is a window of transitional change from the early more variable breathing (P0-P7) to later less variable breathing pattern during $\mathrm{P} 15-$ 
P21. Finally, the third week represents changes leading to adolescence and respiratory control similar to that of an adult. Although there are changes in cytochrome oxidase, respiratory control, neurotransmitters, and neural function, the most notable changes are usually seen during the P10-P13 period and this period seems to be when chemoreflexes have matured and have a more pronounced effect on breathing pattern (Wong-Riley et al., 2013). However, very little information is available concerning the potential role that morphological changes may have on these developmental windows.

\section{Hypoglossal Motoneurons}

\section{Morphology}

It has been estimated that the hypoglossal nucleus (XII) contains approximately 3500 motoneurons (Lewis et al., 1971). Of the neurons in the XII at least $80 \%$ are motoneurons with a small population of interneurons (Boone and Aldes, 1984; Cooper, 1981; Takasu and Hashimoto, 1988). It has been suggested that the size, shape, and dendritic orientation differentiate motoneurons in different regions of the XII (Aldes, 1995; Boone and Aldes, 1984; Cooper, 1981; Kitamura et al., 1983; McClung and Goldberg, 1999; Odutola, 1976). In the dorsal region MNs appear fusiform, orientated along the mediolateral axis and range from $18-40 \mu \mathrm{m}$ in diameter. Those XII MNs in the dorsal region, which innervate intrinsic muscles are reported as significantly smaller $($ mean $=26 \mu \mathrm{m})$ compared to the MNs innervating extrinsic muscles $($ mean $=30 \mu \mathrm{m})$ (McClung and Goldberg, 1999). In the central portion of the ventral subdivision MNs that innervate intrinsic muscles are on average smaller (mean $=23 \mu \mathrm{m})$ and more globular in shape than the larger more lateral multipolar MNs in the ventral division 
$($ mean $=28.7 \mu \mathrm{m})($ Kitamura et al., 1983). Therefore, as with other nuclei the hypoglossal nucleus has separate pools of motoneurons which innervate specific muscles and the morphological differences may relate to the function of the specific muscle innervated (McClung and Goldberg, 1999). For adults XII MNs are further classified based on dendritic morphology. Dendrites of MNs in the dorsal region can be either be confined to the XII region (internal) or extend beyond the borders of the XII (external) (Odutola, 1976).

Although there have been in vitro studies investigating postnatal development of XII motoneuron morphology demonstrating anatomical changes with age, detailed information on the age-related development is lacking. A small number of studies have studied developmental changes in XII MN morphology, but these have not focused on the functional sensitive window (Carrascal et al., 2005; Refik Kanjhan et al., 2016). Morphological differences have been shown between neonatal and adults as well as corresponding electrophysiological changes (Cameron et al., 1991; Fulton and Walton, 1986; Nunez-Abades et al., 1994, 1993; Nunez-Abades and Cameron, 1995).

\section{Electrophysiology}

The electrophysiology of neurons from certain regions of the brain is well known in adults and to a lesser extent in neonatal animals. Much study has also been given to XII MNs in examining the hyperpolarization-activated current as well as different types sodium and potassium voltage-gated channels (Berger et al., 1996; Cameron and NunezAbades, 2000; Ekberg and Adams, 2006; Yoshimoto et al., 2015; Yu and Catterall, 2003). However, there is little work on the electrophysiology of hypoglossal 
motoneurons (XII MNs) during the neonatal period (P1-P21) with one or two studies (Carrascal et al., 2005; Nguyen et al., 2004; Nunez-Abades et al., 1993). Essentially nothing known about the possible relationship between the electrophysiology and morphology for any age except between biophysical properties for four age groups. The sensitive period does not seem to have been studied, but it would be interesting to know if there are electrophysiological changes relating to this developmental window.

There are a few studies which modeled the electrophysiological behavior of motoneurons using a single compartment model (soma) for the neuron (Engel et al., 1999; Fietkiewicz et al., 2011; Purvis and Butera, 2005). However, there are no studies in which the entire morphology of actual XII MNs were modeled. There is a wealth of data on the expression of various voltage-gated channels and the hyperpolarization-activated current and some work on differences in the expression of specific ion channels such as sodium channels with age from P1-P21 (Beckh et al., 1989; González-Castillo et al., 2017).

\section{Data Analysis}

\section{Traditional}

A variety of morphologic measures such as volume, surface area, diameter, length, branch order, and number of dendrites have been reported in the literature (Fukunishi et al., 1999; Refik Kanjhan et al., 2016; Nunez-Abades et al., 1994; NunezAbades and Cameron, 1995). In the past, most morphological studies simply applied parametric statistics producing graphs showing means and standard deviations sometimes including the data points for each individual neurons (Refik Kanjhan et al., 2016; Nunez- 
Abades et al., 1994; Nunez-Abades and Cameron, 1995). A few studies have employed nonparametric statistics for comparing different groups (Refik Kanjhan et al., 2016). Previous studies have also grouped data from a range of ages such as P1-P3, P5-P9, or P15-P30 without any statistical testing or quantitative rationale (Refik Kanjhan et al., 2016; Nunez-Abades et al., 1994; Nunez-Abades and Cameron, 1995). However, these studies used as few as five to ten neurons for the entire group, a mere one or two animals at each age and a couple of neurons per animal. Therefore, previous studies of XII MN morphology utilized relatively small numbers of neurons across age ranges with very little, if any support for the grouping.

\section{Conventional}

For any parametric statistical test one of the key assumptions is of course, that the data is normally distributed for every group being tested. Another assumption for almost any statistical test is that the data is represented by a unimodal distribution. Although, there are readily available statistical tests and analyses (graphical and numerical) for examining whether a set of data is normally distributed or not as well as identifying what type of distribution represents the data (Ghasemi and Zahediasl, 2012; Le Boedec, 2016), their use in neuron morphology studies have not been reported. Graphical methods such as Q-Q plots, Cullen-Frey (skewness versus kurtosis) plots, and cumulative distributions can allow for visual evaluation of whether the data set is normally distributed (Delignette-Muller and Dutang, 2015). Statistical tests such as the Kolmogorov-Smirnov (K-S), Shapiro-Wilk test, Anderson-Darling test, Cramer-von Mises test, and the JarqueBera test can determine the statistical significance of a data set being normally distributed 
(Ghasemi and Zahediasl, 2012; Le Boedec, 2016). There is also one test for determining whether a data set is unimodally distributed or not. The Hartigan dip-test provides a numerical evaluation of how well the data distribution fits a unimodal distribution (Hartigan and Hartigan, 1985). There are in addition several methods for non-parametric ANOVA and multiple comparison tests (García et al., 2010). The conventional battery of tests and statistical methods pertaining to non-parametric data (non-normally distributed) easily allows for thorough testing and evaluation of data regardless of the nature of the distribution and assumptions that the data satisfies. Although sample size is still important the variety of non-parametric tests allows for selection of the appropriate test in respect to the data. One other advancement in statistical analysis is the ability to examine and test for different distributions assessing what type of distribution (normal, lognormal, gamma, Weibull, etc...) best fits the data (Delignette-Muller and Dutang, 2015).

\section{Distribution Analysis}

In addition to comparative tests and validating their assumptions, the type of distribution which best represents the data for each group in an experiment can be valuable in understanding and interpreting the results. There are number of graphical techniques as mentioned above to evaluate the data relative to a number of different distributions (Delignette-Muller and Dutang, 2015). There are also programs such as FitDist which can be used to fit a data set to a specific distribution providing a measure of the "goodness-of-fit" for that distribution. These programs usually also provide both graphical and numerical results comparing the data set to a specific single unimodal distribution. Knowing that two sets of data have different distributions (such as log- 
normal versus gamma) can have a profound influence on the type of comparative statistical tests (such as using the Kolmogorov-Smirnov test) to be employed as well as indicating that the two sets of data may represent different stochastic processes.

Data which may be represented by more than one overlapping distributions is also important in that it suggest that the data is more complex than is represented by a single distribution. Finite mixture modeling is a methodology for examining and testing different multi-distribution models and determining the "goodness-of-fit" for each model (Everitt, 1996). Finite mixture modeling allows the data to be fitted to two or more distributions of a specific type such as normal, log-normal, or gamma while providing a measure of the "goodness" of that fit. A finite mixture model is simply a weighted sum of a number $(\mathrm{N})$ of a specific type of distribution given by the equations

$$
\begin{gathered}
f(x)=\sum_{i=1}^{N} w_{i} \cdot f_{i}(x) \\
\sum_{i=1}^{N} w_{i}=1
\end{gathered}
$$

Where $f(x)$ is the sum of the individual sub-distributions, $N$ is the number of distributions, and $\mathrm{f}_{\mathrm{i}}(\mathrm{x})$ is each sub-distribution, and $\mathrm{w}_{\mathrm{i}}$ is the weight $\left(\mathrm{w}_{\mathrm{i}}<1\right)$ for each subdistribution with the sum of the weights equal to $1(100 \%$ of the distribution $f(x))$. Figure 1 illustrates examples of three different distributions resulting from the sum of two normal distributions. Although only one of the three (Figure 1a) is not unimodal (Bimodal), all three would test to be non-normal. As is seen, one of the three results in a very skewed distribution (Figure 1b) while the third results in a distribution with large 
than expected tails as compared to a normal, log-normal, or other single skewed distribution. 


\section{Bi-modal}

Not Normal or Unimodal

A

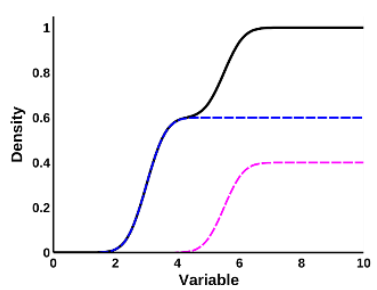

Skewed

Unimodal, but not normal

B

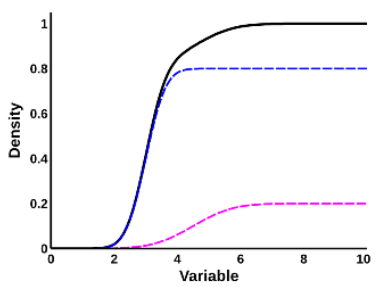

Large tails

Unimodal, but not normal

C

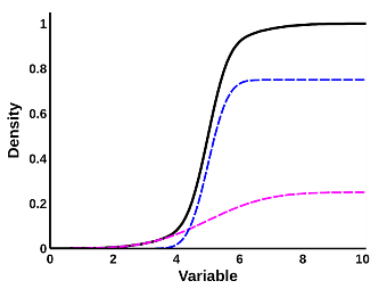

Cumulative Distribution Function (CDF)
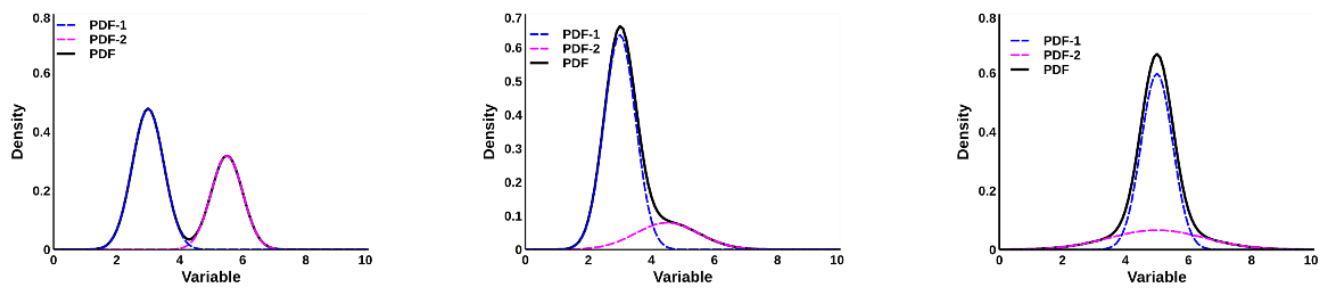

Probability Density Function (PDF)

Figure 1. Graphs illustrating examples of different finite mixture model. The PDF and CDF for mixture models of two normal distributions showing (A) a bimodal distribution, (B) a skewed distribution, and (C) an increase in the tails. The blue and magenta lines represent the two theoretical distributions while the black lines represent the summation of the two distributions. 


\section{Present Study}

In the previous developmental studies of breathing control during the first three weeks post-birth measurements were performed on a daily basis (Liu et al., 2006). However, there are no developmental studies of XII MN morphology with similar temporal resolution (measurements on a daily or semi daily basis). Also, the sensitive period as previously described for respiration has not be examined. Therefore, I performed the present study investigating the morphological changes in XII MNs in a developing rat model. My specific aims were 1) Quantify developmental changes in the morphology of hypoglossal motoneurons in the respiratory region of the brainstem with postnatal age, and 2) Model development changes in the electrophysiological behavior of hypoglossal neurons in the respiratory region of the brainstem as related to age and morphology. Our hypothesis was that there is a morphology change corresponding to the sensitive period that has been shown for neurochemical and respiratory control.

I examine nine separate time points during the first three weeks of development in a rat model to determine the changes in $\mathrm{MN}$ morphology in the hypoglossal nucleus with age. We utilized the Golgi-Cox technique to stain XII MNs to visualize the neurons for morphological analysis at each of the nine separate time points (Figure 2). We also employed conventional statistics and other methodologies to perform detailed analysis of the morphological data. I used nonparametric statistics to compare the nine ages pooling data at ages which were not significantly different. In addition, we utilized the simulation environment NEURON to investigate the potential role of morphology in the electrophysiology of XII MNs with age (Figure 2). Among the innovations of my study were the temporal resolution (individual days), comprehensive statistical (testing for normality and unimodality) and data (finite mixture models) analysis. Another innovation 
was modeling then electrophysiology using actual XII MN morphologies instead of simple single compartment models and models with three none branching dendrites as has been done previously. I simulated previous electrophysiological experiments and compared my results with those experimentally obtained. The last innovation is exploring the possible relationship between XII MN morphology and the passive and active electrophysiological properties. 


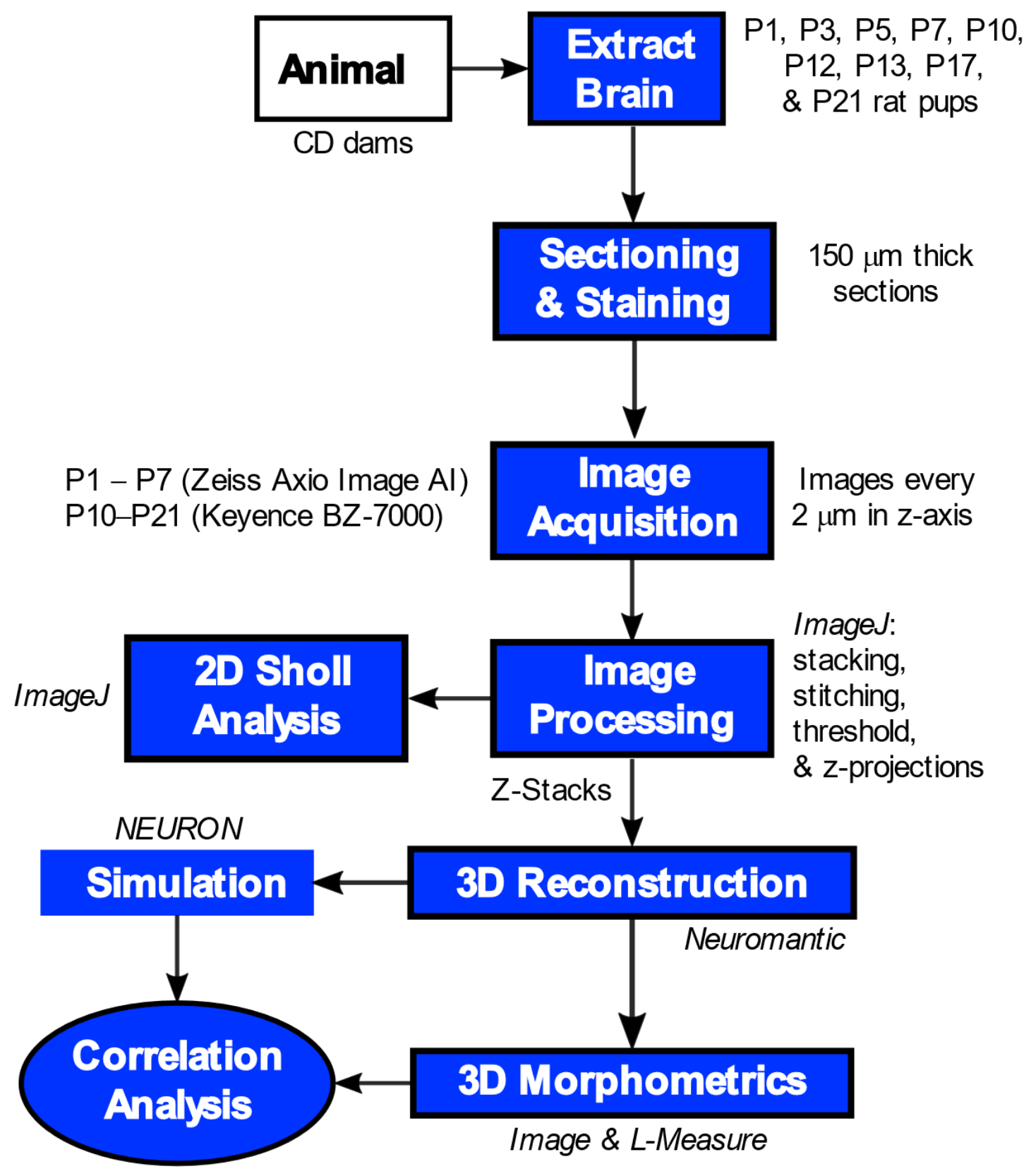

Figure 2. Flowchart of the project showing the morphology, electrophysiology, and overview of the work. 


\title{
CHAPTER TWO
}

\section{CHANGES IN THE MORPHOLOGY OF HYPOGLOSSAL MOTOR NEURONS IN THE BRAINSTEM OF DEVELOPING RATS}

\author{
Paul Allen Williams ${ }^{1,2}$, Denise L. Bellinger ${ }^{1,3}$, Christopher G. Wilson ${ }^{1,2}$ \\ ${ }^{1}$ Division of Physiology, Basic Science Department \\ ${ }^{2}$ Lawrence D. Longo, MD Center for Perinatal Biology, \\ ${ }^{3}$ Department of Pathology and Human Anatomy \\ Loma Linda University School of Medicine \\ 11234 Anderson St, Loma Linda, California 92350
}

The Anatomical Record, 2018, In press

(Modified version) 


\begin{abstract}
The autonomic brainstem generates breathing rhythm by integrating inputs from chemo- and mechanosensors in the viscera and coordinating descending outputs from higher structures in the central nervous system. Hypoglossal motoneurons (XII MNs) receive inputs from respiratory premotor neurons, important for maintaining airway patency. Previous studies in rodents report significant changes in breathing control during the first three weeks of life, with a sensitive period at 10 to 13 days post-birth (P10-P13) characterized by pronounced changes in neurotransmitters, excitation-inhibition balance, and breathing physiology. However, age-dependent morphological changes of XII MNs during the first three weeks post-birth and especially this sensitive period are understudied. Here, we comprehensively characterize and quantify the early morphological changes in rat XII MNs. We hypothesized that there are morphological changes in the soma size and dendritic tree complexity of XII MNs corresponding to the functionallydefined sensitive period observed at postnatal day 10-13 (P10-P13). To test this hypothesis, we used an innovative contemporary statistical approach to analyze GolgiCox stained XII MNs at nine postnatal ages between P1-P21. Our findings reveal two sub-populations of XII MNs, which are dependent on age and morphological feature. Soma size increased $\sim 40 \%$ from $\mathrm{P} 1$ to $\mathrm{P} 21$, without changing shape. However, dendritic arborization increased in extent/distance and complexity. Dendritic branching of developing neurons significantly increased from P1 through P13, with the greatest increase at P10-P13 based on the Sholl method. Our detailed characterization of XII MN morphological development establishes a foundation for the study and elucidation of morphological changes caused by maternal and perinatal conditions.
\end{abstract}




\section{Introduction}

Respiration is a fundamental autonomic behavior that requires coordination of the diaphragm, intercostal muscles, upper airway, and tongue to ensure breathing matches metabolic need. Even though the control of breathing is fundamental to higher organisms, our knowledge of the underlying neural circuitry that generates breathing rhythm is still nascent (Feldman et al., 2013; Feldman and Del Negro, 2006). Importantly, we do not yet understand how breathing regulation and control are influenced by anatomical structure and synaptic remodeling of neurons within the central respiratory circuitry early in development (Feldman et al., 2013; Feldman and Del Negro, 2006; Wong-Riley et al., 2013). The neural network in the brainstem that generates, shapes, and transmits breathing motor pattern must be functional prior to birth (Thoby-Brisson et al., 2005) and continues to develop and remodel in the first weeks of life (Harper and Kinney, 2010; Kinney, 2009; Wong-Riley and Liu, 2005). If the breathing neural network fails to generate a physiologically appropriate rhythm during early postnatal life, pathological breathing patterns can occur — such as apnea of prematurity, periodic breathing, and sudden infant death syndrome (SIDS) with impaired gas exchange that could threaten the life of the newborn (Erickson et al., 2007; Kinney, 2009; Martin and Wilson, 2009).

Brainstem regions such as the nucleus tractus solitarii (nTS), preBötzinger Complex (pBC), and hypoglossal motor nucleus (XII) are necessary for the generation and transmission of breathing rhythm and modification of breathing pattern in response to sensory input (Lindsey et al., 2012; Wong-Riley and Liu, 2005). Early work by other investigators has shown that development of the respiratory network plays a key role in changing breathing pattern (Liu et al., 2006; Mortola, 1984). Understanding how the breathing neural network is remodeled during early life is key to elucidating the synergy 
between morphology and the transmission of breathing rhythm in the perinatal period.

Other reports have shown that neural network behavior is influenced by the morphology of neurons, and that connectivity is altered due to changes in neurotransmitter release and receptor up- and down-regulation (Ferrante et al., 2013; Komendantov and Ascoli, 2009). Hypoglossal motoneurons (XII MNs) supplying the tongue and upper airways are used in in vitro models to provide an index of fictive inspiratory transmission (Koizumi et al., 2008; Purvis and Butera, 2005; Smith et al., 1991, 2000), and our laboratory has previously shown age-dependent changes in the distribution of drive latencies in hypoglossal motoneuron output (Fietkiewicz et al., 2011).

Within the past two decades developmentally-related changes in breathing physiology have been characterized. During the first weeks after birth, changes in cytochrome oxidase occur in the pBC, nTS, XII, and other nuclei of the brainstem (Liu and Wong-Riley, 2001; Wong-Riley and Liu, 2003). Additionally, during postnatal days 10 through 13 there are increases in inhibitory drive, mediated by GABA and glycine, and decreased glutamatergic excitatory drive (Liu and Wong-Riley, 2002, 2006; WongRiley and Liu, 2005). Ventilatory changes have been described from P0 to P21 with an increase in the hypoxic ventilatory response at P0-P6 (Liu et al., 2006, 2009). At P10P21 there is a decrease in overall breathing frequency, but an increase in tidal volume (Liu et al., 2006, 2009). Postnatal days 10-P13 have been referred to as a "sensitive period" in which respiratory development exhibits pronounced changes in frequency and variability of breathing pattern. This "sensitive period" is an intermediate window of transition from the early more variable breathing (P0-P7) to later less variable breathing pattern seen during P15-P21. Finally, the third week represents changes leading to 
adolescence and respiratory control similar to that of an adult. Although changes in cytochrome oxidase, respiratory control, neurotransmitters, and neural function, occur during the P10-P13 period, a major component of the breathing changes are likely due to the maturation of chemoreflexes (Wong-Riley et al., 2013). However, little information is available concerning the potential role that morphological changes may have on these developmental windows.

Morphological and electrophysiological differences have previously been shown between neonate and adult animals (Cameron et al., 1991; Fulton and Walton, 1986; Nunez-Abades et al., 1993, 1994; Nunez-Abades and Cameron, 1995). Although in vitro studies investigating postnatal development of XII motoneuron morphology showed anatomical changes with age, a detailed description of age-related development is lacking. The few developmental studies investigating changes in XII MN morphology (Carrascal et al., 2005; Kanjhan et al., 2016a) did not focus on the functionally sensitive window, P10-P13. Previous studies have collapsed data across age ranges or windows based on arbitrary grouping (Kanjhan et al., 2016a), or omitted certain windows or age ranges, which have been indicated as important in respiratory control, particularly P10P13 (Nunez-Abades et al., 1994; Nunez-Abades and Cameron, 1995). The ages we studied are based on postnatal days when significant respiratory and neurotransmitter changes have previously been reported in the developing brainstem of rats (Liu et al., 2006; Wong-Riley and Liu, 2005). Previous studies have suggested that there is little if any difference in normal development between genders for the ages investigated in this study (Kanjhan et al., 2016a; Wong-Riley et al., 2013), thus we did not differentiate between genders. 
Our aim in these experiments was to study the maturation of XII MNs within the context of previously reported physiological and neurochemical changes around postnatal days 10 to 13 . Our objective in this study was to comprehensively characterize and quantify the somatic and dendritic morphological features of XII MNs across the first three weeks of life. We hypothesized that there are morphological changes in XII MNs which correspond to the functionally-defined sensitive period observed at P10-P13. We tested this hypothesis using Golgi-Cox staining to visualize neuronal morphology for quantitative examination of somata and dendritic arbor of developing XII MNs at nine separate postnatal ages from P1-P21 in a rat model. We employed two-dimensional (2D) Sholl analysis to quantify morphological metrics including the maximum number of intersections, radius at which the maximum number of intersections occur, ramification index, and enclosing radius for the dendritic tree of XII MNs from P1 to P21. We tested for both normality and unimodality of the data distribution as well as nonparametric statistics and contemporary statistical methodology including finite mixture distribution models to determine how the data was distributed. Previous studies have not comprehensively reported the use of normality or unimodality testing of their data, so applying contemporary statistical methods to our data has the potential of revealing a greater understanding of XII MN development during the first three weeks post-birth.

\section{Materials and Methods}

\section{Animals}

Pregnant Sprague-Dawley dams (Charles River Laboratories International, Inc., Wilmington, MA) obtained approximately 18 days after the start of pregnancy (E18) were allowed to deliver naturally in the Loma Linda University Animal Care Facility. To 
determine how many time points to use a minimal litter size of 9 rat pups was assumed, therefore we selected nine postnatal ages across the first three weeks of development for this study. To adequately reflect the early period of development when several changes occur P1, P3, P5, and P7 were chosen (Liu et al., 2006; Liu and Wong-Riley, 2002, 2006, 2001; Wong-Riley and Liu, 2005). For the second week the sensitive developmental period was the focus, so P10, P12, and P13 were chosen. This only left two time points for the third week of development, so we chose P17 and P21 as the period of maturation prior to the adult respiratory pattern. As a result, rat pups at nine postnatal ages $(\mathrm{P} 1, \mathrm{P} 3$, P5, P7, P10, P12, P13, P17, and P21 days) were used in the study. Typical litter size was approximately 9-14 pups (Table 1) and no distinction was made between genders. When two litters were used they were balanced if needed so that the number of pups per litter were similar if not the same. All procedures and protocols used in this study were approved by the Institutional Animal Care and Use Committee at Loma Linda University and followed the guidelines by the National Institutes of Health Guide for the Care and Use of Laboratory Animals (http://www.ncbi.nlm.nih.gov/books /NBK54050).

\section{Golgi-Cox Staining}

On the appropriate developmental day, animals were weighed, euthanized, and the brain extracted. For postnatal ages P1 through P7 the pups were anesthetized with isoflurane (Western Medical Supply). When unresponsive to toe pinch, their brains were rapidly extracted and placed in chilled $0.9 \%$ saline. For postnatal ages P10 through P21, animals were placed in an induction chamber with oxygen (100\%) flow at $0.65 \mathrm{~L} / \mathrm{min}$ and isoflurane administered at a concentration of 3 to $4 \%$ based on the postnatal age. After the animal was unresponsive to toe pinch it was removed from the induction 
chamber and placed on a platform with the oxygen and isoflurane continuously administered via a nose cone. The animal was perfused with $0.9 \%$ saline for approximately two minutes and then the brain was removed. For ages P1 through P7, the whole brain was maintained intact while for P10 through P21 the brain was sectioned coronally into two tissue blocks.

After removal, the brains were immediately transferred from $0.9 \%$ saline to impregnation solution from a commercial kit (superGolgi Kit, Bioenno Tech, LLC, Santa Ana, California). Impregnation solution was changed after two days then the specimens were left in the solution for five more days according to the supplier's instructions and previous reported methods (Das et al., 2013). Sections impregnated at different time durations showed that the best results were obtained with 7 days of impregnation changing the solution after the initial two days. After a total of 7 days in impregnation solution at room temperature, the brains were placed in two changes of post-impregnation buffer at room temperature (changed each day for two days). We performed coronal sectioning of tissue at $150 \mu \mathrm{m}$ with a vibratome (Vibratome Series 1000 Classic-Tissue Sectioning System, Leica, St. Louis) (Gibb and Kolb, 1998). Sections were mounted onto gelatin-coated slides and allowed to dry slowly in a humidified chamber overnight (Das et al., 2013). The slides were then allowed to dry at room temperature and humidity for approximately one hour and then rehydrated in phosphate buffered saline, stained, placed in post-staining buffer, and then washed, dehydrated, and cleared in xylene as per the supplier's instructions and previous reported methods (Das et al., 2013). After staining, Permount (Fisher Scientific, SP-15) was applied to the slides which were then coverslipped and allowed to fully dry. 


\section{Imaging}

All sections were imaged using a $2 \mathrm{x}$ objective and compared with atlases (Khazipov et al., 2015) corresponding to the appropriate postnatal period to identify the sections containing the XII nucleus from each animal (Figure 3). We used a 40x objective to acquire image stacks for each XII motor neuron. For the P1-P7 animals single image stacks with one image every $2 \mu \mathrm{m}$ in the z-direction were obtained using a microscope (Ziess Axio Imager, Carl Zeiss Microscopy, LLC One Corporation Way 1 Peabody, MA USA) with a motorized stage (Applied Scientific Instrumentation, Eugene, OR) and imaging software (Stereologer, Stereology Resource Center, Tampa FL). For the P10P21 animals, we acquired single and multiple stacks with one image every $2 \mu \mathrm{m}$ in the zdirection. Multiple z-stacks are required for many of the P10-P21 animals in order to image the entire dendritic morphology of a single neuron at 40x, so we used another microscope (Keyence, BZ-9000, Itasca, IL) to capture the arborization for these neurons. The resolution for the Ziess Axio Imager was $0.402 \mu \mathrm{m} /$ pixel while for the Keyence the resolution was $0.271 \mu \mathrm{m} /$ pixel.

\section{Morphological Measurements}

After the images were processed in ImageJ (https://imagej.nih.gov/ij/), the Sholl analysis plug-in (Ferreira et al., 2014) was used to perform 2D measurements. For the P1 through P7 animals, image stacks were converted to z-projections, thresholding was applied to the z-projections, and single neurons with minimal or no overlapping dendrites and minimal artifacts were selected, isolated, and measured. For the P10 through P21 animals, single image stacks were processed the same as in P1 through P7 animals, but 
multiple stacks were stitched together using the ImageJ stitching plug-in (Preibisch et al., 2009). Based on power analysis ( $G^{*}$ Power, http://www.gpower.hhu.de/en.html) we determined that approximately 36 XII MNs (in each postnatal age group) would provide sufficient statistical power. We obtained at least 5-7 pups per age from seven litters. Litter size ranged from 9 to 14 (average of about 12 pups per litter, Table 1). A total number of 30 or more neurons were obtained for each postnatal age (Table 2). The morphology of the soma was measured by determining the area, perimeter, minor diameter, and major diameter based on the approximate border of the soma. From these measurements the equivalent circular diameter $\left(\mathrm{ECD}=[4 \mathrm{x} \text { Area/ } \pi]^{1 / 2}\right)$, form factor $(\mathrm{FF}=$ $(4 \pi \times$ Area $) /$ Perimeter $\left.^{2}\right)$, and aspect ratio $(\mathrm{AR}=$ maximum diameter/ minimum diameter $)$ of the soma were calculated (Fukunishi et al., 1999; Standard Practice for Characterization of Particles, 2003). Two-dimensional Sholl analysis (Ferreira et al., 2014; Sholl, 1950) was performed by creating concentric circles at $10 \mu \mathrm{m}$ increments from the soma and then the number of dendrites intersecting these circles were counted. From these counts Sholl profiles are generated as the number of intersections at each circle and the following parameters are determined: maximum number of intersections (max intersections), radius at which maximum number of intersections occur (max intersection radius), ramification index (the ratio between max intersections and the number of primary branches), and enclosing radius (the largest radius at which a dendrite intersects) (http://imagej.net/Sholl_Analysis). 
Table 1. Number of animals available for measurement per litter for the nine postnatal ages. The numbers in parentheses are the number of pups born in that litter, but usually only 9 to 12 pups in a single litter were used. Animals in which the specimen or sections were lost are designated by N/A.

\begin{tabular}{|c|c|c|c|c|c|c|c|c|}
\hline \multirow{2}{*}{$\begin{array}{l}\text { Age } \\
\text { (days) }\end{array}$} & \multicolumn{7}{|c|}{ Litter } & \multirow{2}{*}{$\begin{array}{c}\text { Total \# } \\
\text { of } \\
\text { Animal } \\
\text { s }\end{array}$} \\
\hline & $\begin{array}{c}1 \\
(\mathrm{~N}=12)\end{array}$ & $\begin{array}{c}2 \\
(\mathrm{~N}=9)\end{array}$ & $\begin{array}{c}3 \\
(\mathrm{~N}=12)\end{array}$ & $\begin{array}{c}4 \\
(N=10)\end{array}$ & $\begin{array}{c}5 \\
(N=14)\end{array}$ & $\begin{array}{c}6 \\
(N=14)\end{array}$ & $\begin{array}{c}7 \\
(\mathrm{~N}=10)\end{array}$ & \\
\hline P1 & N/A & N/A & 2 & 1 & 1 & N/A & 2 & 6 \\
\hline P3 & N/A & 1 & 1 & 1 & 1 & 1 & 2 & 7 \\
\hline P5 & 1 & 1 & N/A & 1 & 2 & 1 & N/A & 6 \\
\hline P7 & N/A & 1 & 1 & 1 & 1 & 2 & N/A & 6 \\
\hline P10 & 1 & 1 & 1 & 1 & 2 & 1 & N/A & 7 \\
\hline P12 & 1 & 1 & 1 & 1 & 1 & 1 & 1 & 7 \\
\hline P13 & 1 & 1 & 1 & 1 & 1 & 1 & N/A & 6 \\
\hline P17 & N/A & N/A & 1 & 2 & 1 & 2 & N/A & 6 \\
\hline P21 & 1 & 1 & 1 & 1 & $\mathrm{~N} / \mathrm{A}$ & N/A & 1 & 5 \\
\hline Total & 5 & 7 & 9 & 10 & 11 & 8 & 6 & 56 \\
\hline
\end{tabular}



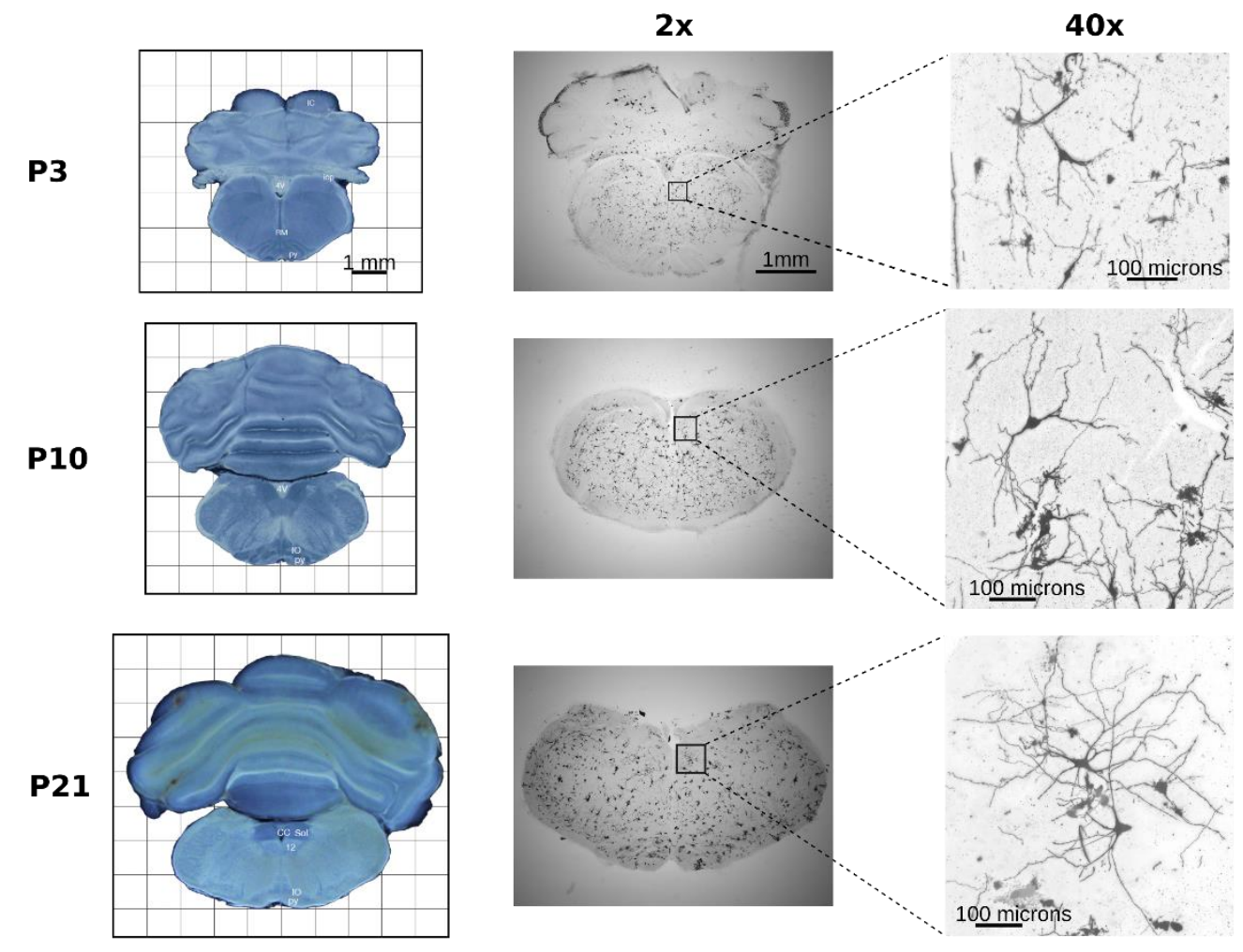

Figure 3. Morphology of hypoglossal motoneurons for the rat pups at P3, P10, and P21 postnatal ages. Shown are the images of the corresponding atlas (Left) for P3, P10, \& P21 [R. Khazipov et al., 2015], as well as the low magnification (2X) micrographs of the sections (center), and high magnification (40X) images of the Golgi-Cox stained hypoglossal motoneurons. The high magnification image for the $\mathrm{P} 3$ is a single stack while for the P10 \& P21 the high magnification images are six stacks stitched together as described in the methods. 
Table 2. Number of XII MN's analyzed (per litter) for the nine postnatal ages. For ages and litters in which the specimen or sections were lost are designated by N/A.

\begin{tabular}{lrrrrrrrrr}
\hline $\begin{array}{l}\text { Age } \\
\text { (days) }\end{array}$ & \multicolumn{1}{c}{$\mathbf{1}$} & $\mathbf{2}$ & $\mathbf{3}$ & $\mathbf{4}$ & $\mathbf{5}$ & $\mathbf{6}$ & \multicolumn{1}{c}{ Litters } & $\begin{array}{l}\text { Total \# of } \\
\text { Neurons }\end{array}$ \\
\cline { 2 - 7 } P1 & N/A & N/A & 18 & 3 & 8 & N/A & 19 & 48 \\
P3 & N/A & 5 & 6 & 8 & 17 & 7 & 15 & 58 \\
P5 & 9 & 4 & N/A & 6 & 22 & 6 & N/A & 47 \\
P7 & N/A & 9 & 10 & 7 & 10 & 25 & N/A & 61 \\
P10 & 19 & 6 & 9 & 10 & 13 & 5 & N/A & 62 \\
P12 & 4 & 6 & 5 & 7 & 4 & 24 & 8 & 58 \\
P13 & 3 & 6 & 13 & 13 & 11 & 5 & N/A & 51 \\
P17 & N/A & N/A & 9 & 21 & 6 & 8 & N/A & 44 \\
P21 & 5 & 8 & 6 & 6 & N/A & N/A & 6 & 31 \\
\hline Total & 40 & 44 & 76 & 81 & 91 & 80 & 48 & 460 \\
\hline
\end{tabular}




\section{Location and Selection of Neurons}

\section{Mapping of Neuron Locations}

Figure 3 shows photomicrographs from atlases (Khazipov et al., 2015) of P3, P10, and P21 (left panel) with low (2x) and high (40x) magnification images of Golgi-stained XII MNs representative sections (center and right panels, respectively). The estimated lambda for the low magnification images was used to match the images to the atlas image. The position of each XII motoneuron was measured with ImageJ to acquire the length and angle of a line from the apex of the fourth ventricle (rostral) or central canal (caudal). Then the length and angle were converted to $\mathrm{x}$ (horizontal) and y (vertical) distance with the $\mathrm{x}$ representing the medial-lateral and the $\mathrm{y}$ representing the dorsalventral direction. These points were projected on a plot relative to this anatomical reference point and the boundary of XII determined in a similar manner, as previously described (Kanjhan et al., 2016a; Koizumi et al., 2008). Points from both left and right hemispheres were mapped to one side of the section for the purpose of determining the total number and location of neurons in each section. For the rostral-caudal extent of XII, sections were matched with a representative rat atlas for each postnatal age (Khazipov et al., 2015) and the lambda coordinate along with the y distance (dorsal-ventral direction) was used to project the point in a sagittal plane similar to that previously described (Kanjhan et al., 2016a). Due to size differences between the postnatal ages, lambda was normalized by the lambda value at the obex. Neurons located in the XII nucleus for both the coronal and sagittal maps were measured while any neurons outside the XII were excluded from analysis. 


\section{Selection of Motoneurons}

Although the majority of neurons in the XII nucleus are motoneurons, there are a few interneurons (Boone and Aldes, 1984; Cooper, 1981; Takasu and Hashimoto, 1988). Because of this we needed criteria to determine if the measured neuron was a motoneuron. In the adult rat motoneurons are larger in diameter $(19-50 \mu \mathrm{m})$ than the interneurons (10-18 $\mu \mathrm{m})$ (Boone and Aldes, 1984; Cooper, 1981; Takasu and Hashimoto, 1988). For mouse XII MNs it has been shown that there was approximately a $25 \%$ decrease in major diameter at P1-P4 as compared with P14-P28 (Kanjhan et al., 2016a). Adult interneurons have been described as fusiform or oval in shape with few primary dendrites from both poles and from the center, while motoneurons were multipolar in shape (Boone and Aldes, 1984; Takasu and Hashimoto, 1988). However, this has not been as well established for neonatal XII neurons (Kanjhan et al., 2016a; Nunez-Abades et al., 1994; Nunez-Abades and Cameron, 1995). Therefore, our main criteria for determining a motoneuron was that from P1 to P7 the size needed to be at least $18 \mu \mathrm{m}$ and at least $20 \mu \mathrm{m}$ at P10 to P21. The shape (oval, fusiform, multipolar) of the neuron as previously described was used as a secondary criterion as needed (Boone and Aldes, 1984; Cooper, 1981; Fukunishi et al., 1999; Kanjhan et al., 2016a; Nunez-Abades et al., 1994; Nunez-Abades and Cameron, 1995; Takasu and Hashimoto, 1988).

\section{Data Analysis}

\section{General Analysis and Comparisons}

For all neurons at each postnatal age, individual Sholl profile measurements were averaged and plotted with their standard deviation (Std). Cumulative distributions of 
selected parameters were plotted to examine the distribution shape of that parameter for each age of interest. Box plots with median and quartiles were also graphed for ready comparison. The Shapiro-Wilk test for normality (Ghasemi and Zahediasl, 2012; Le Boedec, 2016) and Q-Q plots were used to determine if the data were normally distributed. Hartigan's dip test (Hartigan and Hartigan, 1985) was used to assess unimodality of the data (diptest package in R; https://cran.r-project.org/web/packages/ diptest/index). For normality and unimodality, statistically significant differences were set at the $\mathrm{p}<0.01$ level. Non-parametric one-way analysis using the Kruskal-Wallis test was performed on morphological measures and non-parametric pairwise post-hoc testing using Dunn's test for multiple comparisons (PMCMR package in R; https://cran.rproject.org/web/packages/PMCMR/) with statistical significance set at the $\mathrm{p}<0.05$ level (García et al., 2010). For the non-parametric comparison, a difference at $\mathrm{p}<0.05$ level was considered sufficient, but to conclude that data was not normally or unimodally distributed (key assumptions for statistics) a $\mathrm{p}<0.01$ level was chosen.

\section{Distribution Analysis}

For cases in which the data was not unimodal, finite mixture distribution models were employed to fit the data to more than a single distribution (Everitt, 1996; Forbes et al., 2011). For morphometric parameters that were non-normally distributed, distribution analysis (fitdistrplus package in R; https://cran.r-project.org/web/packages/fitdistrplus /index.html) was used to determine whether a single distribution provided a good-fit (Delignette-Muller and Dutang, 2015). Non-normally distributed data was examined using Q-Q plots for different distributions such as log-normal or gamma as well as 
Cullen-Frey (skewness/kurtosis) plots which also show data relative to normal, lognormal, and gamma distributions. Goodness of fit measures for several distributions were generated for the data to determine quantitatively which type of distribution best fit the data. For cases in which a single distribution fit was not found, finite mixture distribution models (mixdist package in R; https://cran.r-project.org/web/packages/mixdist/ index.html) were employed to fit the data to more than a single distribution. The distribution model was chosen on goodness-of-fit and the consistency of the model to fit across several parameters and age ranges. All statistical analyses were performed using $\mathrm{R}$ (https://www.r-project.org/).

\section{Results}

The mean body weights of animals for each postnatal age are shown in Figure 4. Rat pup body weight increased relatively linearly with postnatal age. The coefficients of variation $(\mathrm{CV}$; inset Figure 4$)$ for the different ages were $\leq 17 \%$. We measured 460 neurons in 56 pups (Table 1 and Table 2). Topographical maps of the measured XII neurons created for P1, P3, and P5 neurons (Figure 5A), P7, P10, and P12 (Figure 5B), and P13, P17, and P21 (Figures 5C) for both the medial-lateral (coronal) and rostralcaudal (sagittal) orientations showed that selected XII neurons for each postnatal day were essentially uniformly distributed in the medial, lateral, dorsal, ventral, rostral, and caudal extent of the XII nucleus. Photomicrographs of P3, P12, and P21 XII MNs (Figure 6) illustrated dendritic spines (Figure 6A, 6B, and 6C), which were observed at all ages. Dendrites extending across the midline were also observed at P3 (Figure 6A) and all other ages. Dendrites extending beyond the border of the XII nucleus were observed at P21, (Figure 6D) as well as the other ages. Additionally, while some XII MNs exhibited 
near symmetry in dendritic branching across the primary branches, in general the dendritic branching for XII MN were asymmetrical across primary branches (Figure 6).

\section{Soma Size, but Not Shape Changes with Age}

Figure 7 shows representative examples of neuron somata for the nine postnatal ages at the same magnification. The images for Figure 7 were selected based on their quantitative values as compared with the corresponding mean and median values for that parameter and postnatal age. Qualitative evaluation of XII MNs suggested a progressive increase in the size of the perikarya, thickness of their proximal dendrites, and an increase in primary dendrites. We also saw that some neurons appeared globular while others were multipolar. Figure 8 shows the cumulative distributions of the size and shape for each of the nine postnatal ages. There was a progressive rightward shift in size distribution of the somata with increasing postnatal age, based on equivalent circular diameters, ECD (Figures 6A) and their major diameter (Figure 8B). However, there was not much change in distribution with shape based on the aspect ratio (Figures 8C) and a small rightward shift in form factor (Figure 8D). The ECD (Figure 8A) and to a lesser extend the major diameter (Figure 8B) seemed to show three groups with P1, P3, and P5 as one group, P7, $\mathrm{P} 10$, and $\mathrm{P} 12$ as a second, and $\mathrm{P} 13, \mathrm{P} 7$, and $\mathrm{P} 21$ as the third group. The aspect ratio (Figure 8C) showed very little if any change while the form factor showed some change (Figure 8D). Some cumulative distributions such as the ECD at P21 (Figure 8A) demonstrated a shape in the distribution that does not appear to represent a normal distribution. 


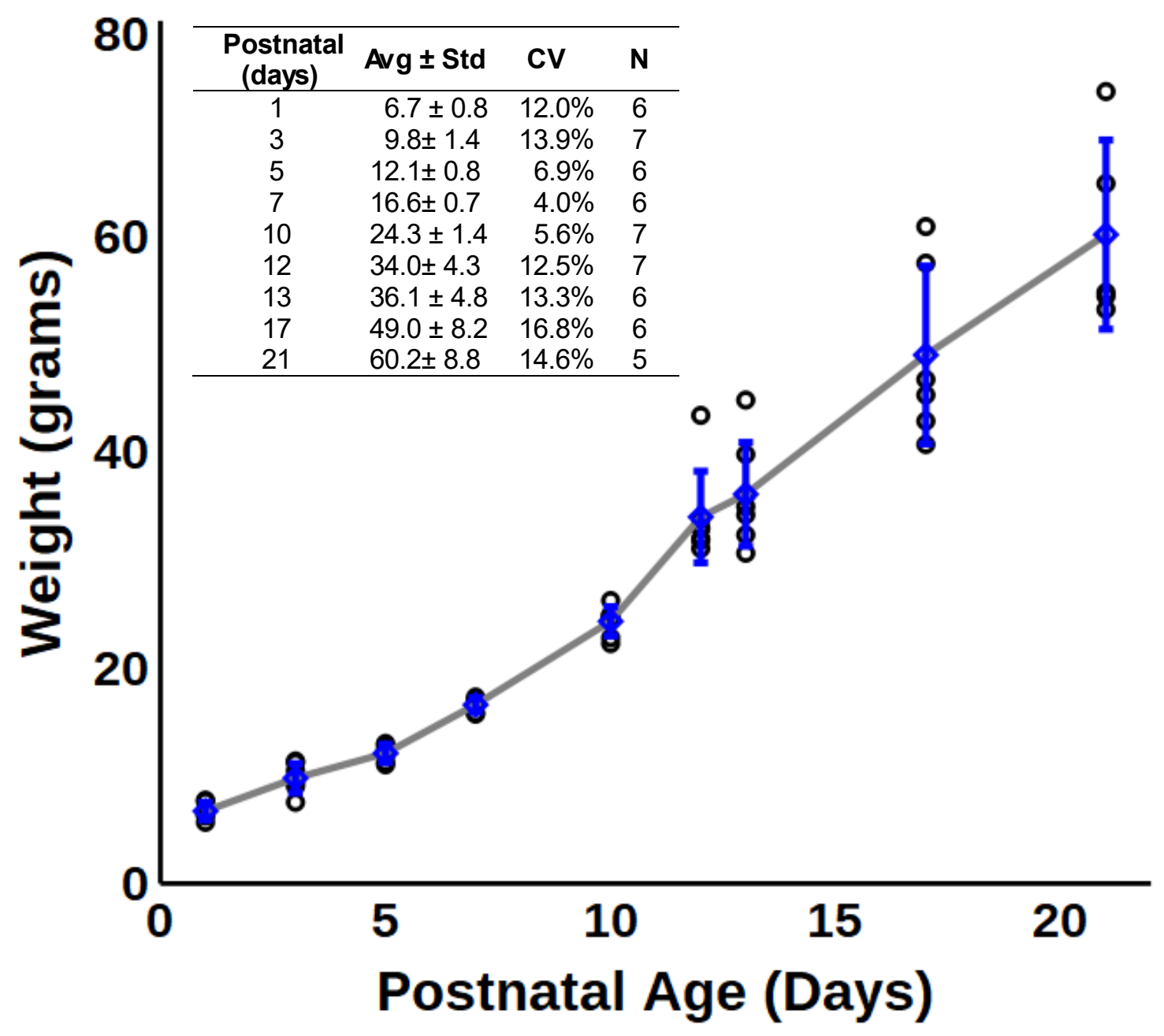

Figure 4. Mean $(\diamond)$ and standard deviation (in blue) of animal body weight at each postnatal age with a gray line connecting the mean values. Weight data for each individual animal is shown as a circle (O), but some values do overlap. A complete listing of the number of animals by age and litter is provided in Table 1 . The coefficient of variance and the number of animals at each postnatal day (age) is provided in the inset. 


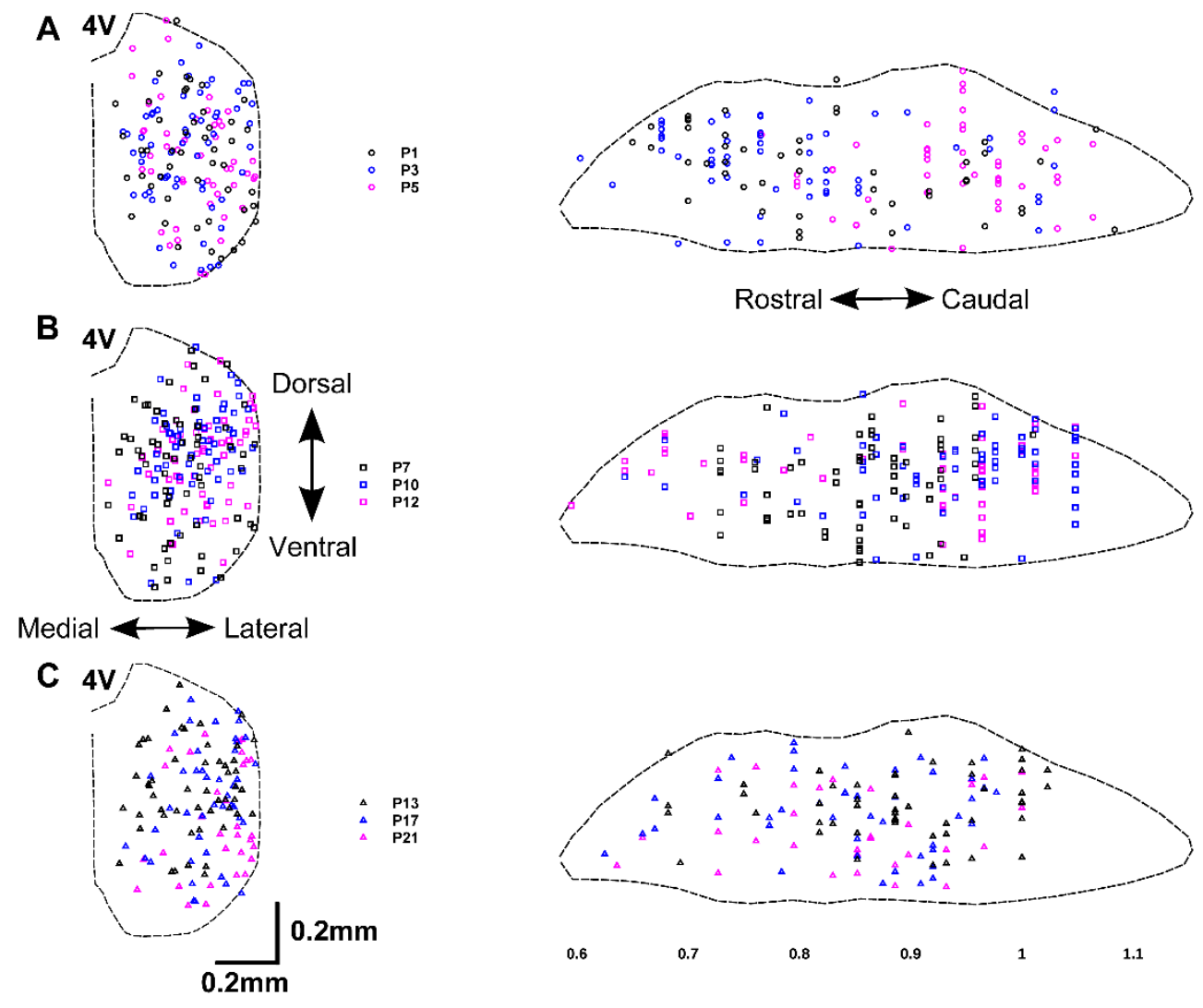

Figure 5. Maps of the location of measured neurons in the dorsal-ventral, medial-lateral, and rostral-caudal directions for a) P1, P3, \& P5; b) P7, P10, \& P12; and c) P13, P17, \& $\mathrm{P} 21$. The number of data points for each chart is given in Table 2 and the hypoglossal region is shown as the dashed region. The coronal maps on the left show the medial-lateral and dorsal-ventral directions while the sagittal maps on the right show the dorsal-ventral and rostral-caudal directions. For the coronal maps the scale bars apply, but for the sagittal maps the rostral-caudal direction is a normalized value of lambda given by the horizontal axis where 1 approximately indicates the obex. 

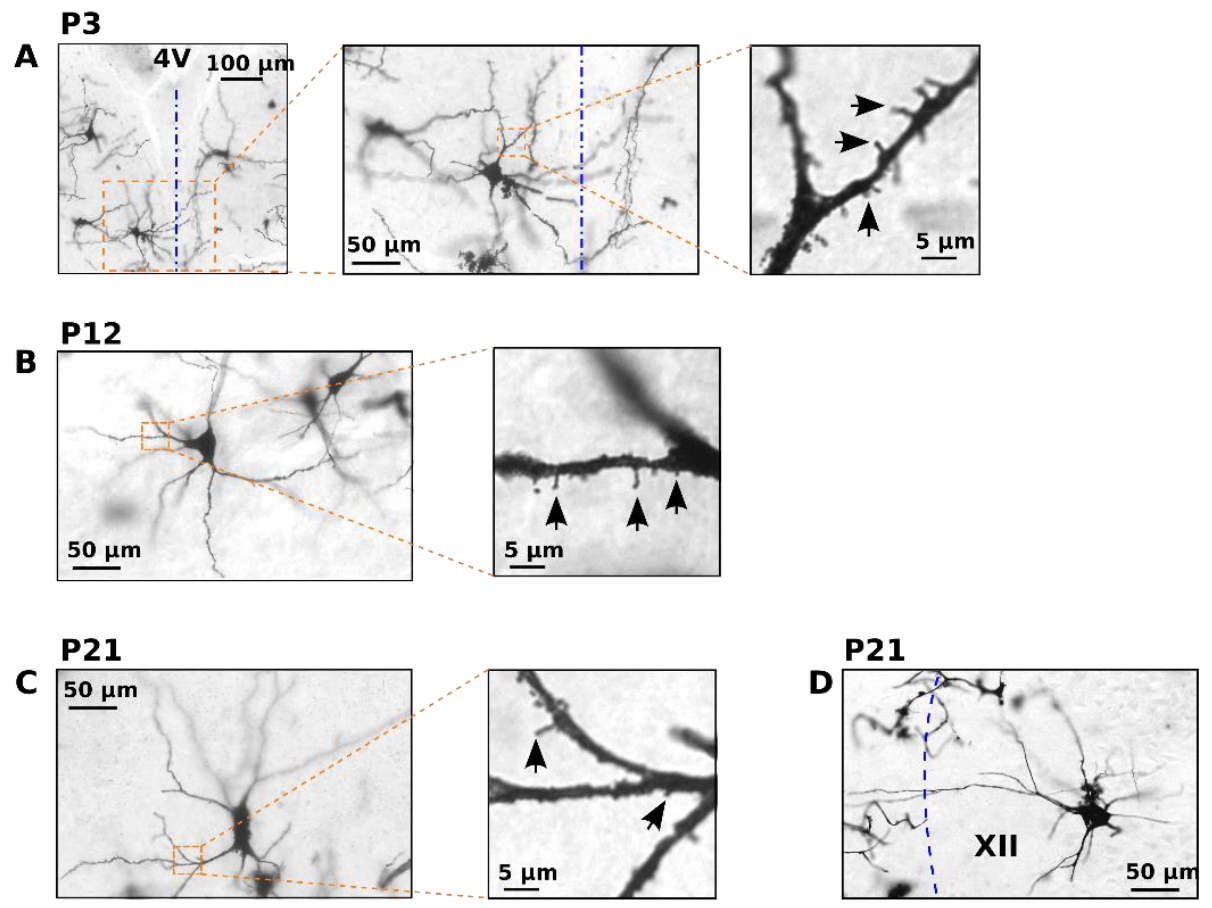

Figure 6. Photomicrographs of XII MNs: a) a P3 neuron illustrating dendrites crossing the midline (vertical dashed line) and dendritic spines (arrows), b) a P12 neuron demonstrating spines (arrows), c) a P21 neuron exhibiting spines (arrows), and d) a P21 neuron showing dendrites extending beyond the border of the XII nucleus (dashed arc). 

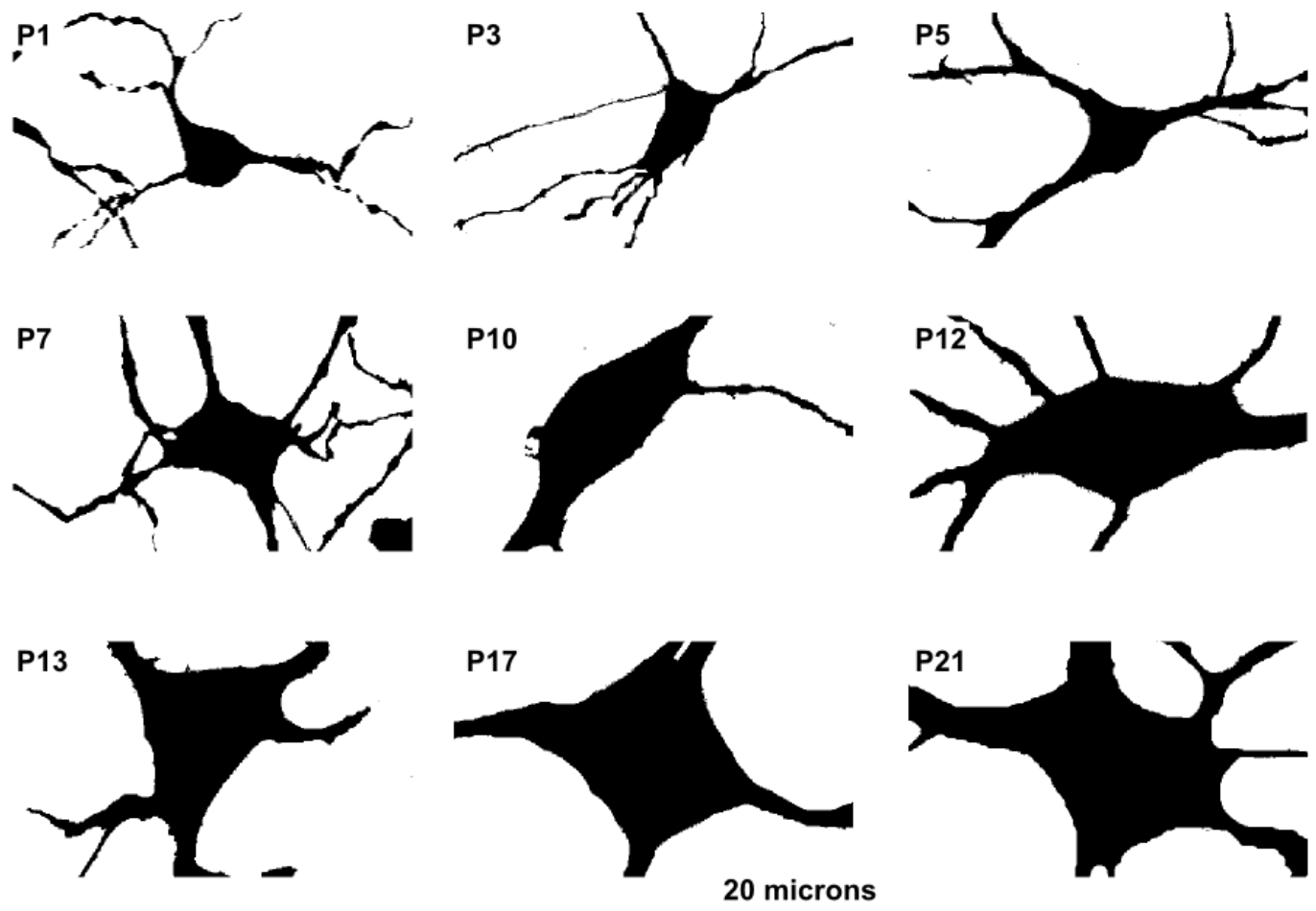

Figure 7. Representative threshold images of various XII MN soma and their respective values for ECD, major diameter, aspect ratio, and form factor. The ECD and major diameter (MajDia) provide information on size while the aspect ratio (AR) and form factor (FF) provide information on the shape of the soma. Images were chosen based on the quantitative values (means \& medians) for the morphometrics. Legend: P1 $-\mathrm{ECD}=18.47$ $\mu \mathrm{m}, \mathrm{MajDia}=21.34 \mu \mathrm{m}, \mathrm{AR}=1.336, \mathrm{FF}=0.695 ; \mathrm{P} 3-\mathrm{ECD}=20.28 \mu \mathrm{m}, \mathrm{Maj} \mathrm{Dia}=29.40$ $\mu \mathrm{m}, \mathrm{AR}=2.102, \mathrm{FF}=0.502 ; \mathrm{P} 5-\mathrm{ECD}=22.55 \mu \mathrm{m}, \mathrm{MajDia}=25.60 \mu \mathrm{m}, \mathrm{AR}=1.289, \mathrm{FF}$ $=0.543 ; \mathrm{P} 7-\mathrm{ECD}=28.87 \mu \mathrm{m}, \mathrm{MajDia}=33.10 \mu \mathrm{m}, \mathrm{AR}=1.315, \mathrm{FF}=0.582 ; \mathrm{P} 10-\mathrm{ECD}$ $=40.06 \mu \mathrm{m}, \mathrm{MajDia}=68.44 \mu \mathrm{m}, \mathrm{AR}=2.918, \mathrm{FF}=0.510 ; \mathrm{P} 12-\mathrm{ECD}=48.37 \mu \mathrm{m}$, MajDia $=80.82 \mu \mathrm{m}, \mathrm{AR}=2.792, \mathrm{FF}=0.524 ; \mathrm{P} 13-\mathrm{ECD}=44.96 \mu \mathrm{m}, \mathrm{MajDia}=54.12 \mu \mathrm{m}, \mathrm{AR}=$ $1.449, \mathrm{FF}=0.358 ; \mathrm{P} 17-\mathrm{ECD}=54.53 \mu \mathrm{m}, \mathrm{MajDia}=56.37 \mu \mathrm{m}, \mathrm{AR}=1.069, \mathrm{FF}=0.413$; $\mathrm{P} 21-\mathrm{ECD}=55.56 \mu \mathrm{m}, \mathrm{MajDia}=67.14 \mu \mathrm{m}, \mathrm{AR}=1.460, \mathrm{FF}=0.494$. The $20 \mu \mathrm{m}$ scale bar for all images is shown at bottom of figure under the P17 XII MN. 

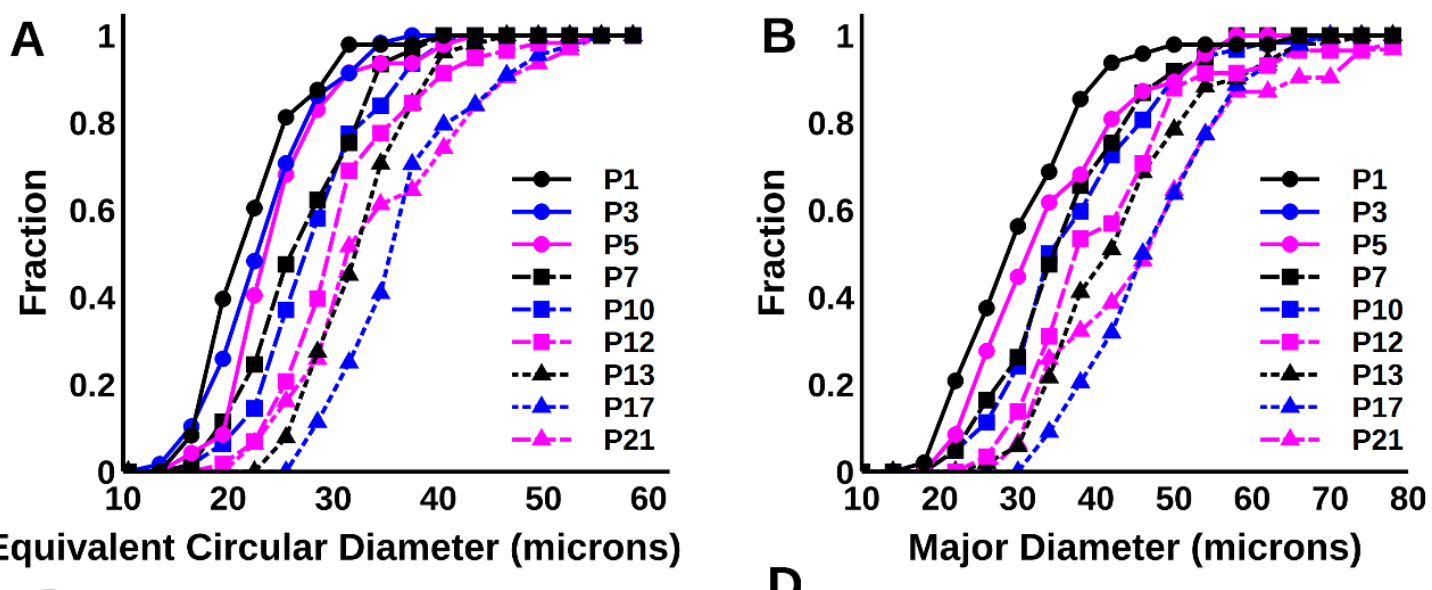

Equivalent Circular Diameter (microns)
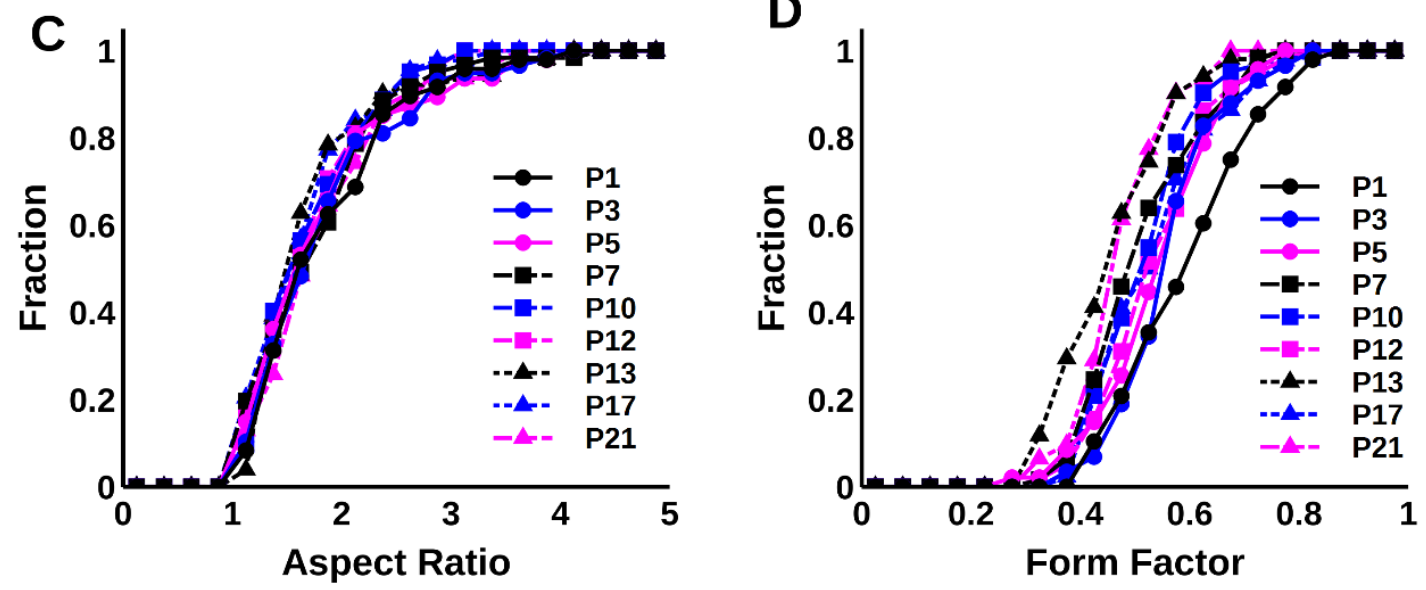

Figure 8. Cumulative distributions of the soma morphology showing the a) equivalent circular diameter, b) major diameter, c) aspect ratio, and d) form factor. The y-axis shows the cumulative fraction such that 0.2 is $20 \%, 0.6$ is $60 \%$, and 1 is $100 \%$. Results from the Shapiro-Wilks test for normality are in Table 3 and all distributions were found to be unimodal as determined by Hartigan's dip test. 
The mean and median for the soma of the XII MNs increased in size with increasing age (Figures 9A and 9B) but did not significantly change in shape (Figures 9C and 9D). The soma size increases linearly, leveling off around P17-P21 (Figures 9A and 9B). The ECD for the P12-P21 ages was significantly larger from those of the P1-P5 neurons by approximately 40\% (Figure 9A). There was also a statistically significant $(p<0.05)$ difference between the ECD of P13-P21 neurons and the P10 neurons with the P13-P21 neurons having approximately a 15\% increase in ECD (Figure 9A). The major diameter of the neurons from rats at P12-P21 were statistically significantly $(p<0.05)$ larger than those from rats at P1-P5 by about the same percent as with ECD (Figure 8B). The aspect ratio (Figure 9C) was not significantly different across any age. However, the form factor (Figure 9D) was significantly lower at P7 and P10 compared with neurons at $\mathrm{P} 1$ with a decrease of about $16 \%$ and $\mathrm{P} 13$ was significantly lower (18\%) than P1, P3, or P5.

\section{Soma Morphology is Unimodal, but not Necessarily Normally}

\section{Distributed}

The Shapiro-Wilk test for normality (Table 3) showed that at specific days (P1, $\mathrm{P} 5, \mathrm{P} 12$, and $\mathrm{P} 17)$ the size distribution was significantly $(p<0.01)$ different from a normal distribution. Although the aspect ratio was significantly different from normal, it is a ratio of two numbers (quotient of two distributed variates) which may result in a non-normal distribution (Marsaglia, 2006). Hartigan's dip test for unimodality suggest that all soma morphometric parameters were not significantly $(p>0.25)$ different from a unimodal distribution. Although all the morphometric parameters were found to be unimodal, 
parameters at one or more postnatal ages were found to be non-normally distributed (Table 3). Therefore, distribution fitting and finite mixture modeling was used to characterize the data distribution. To improve the model fit our data was pooled into three groups 1) P1-P5, 2) P7-P12, and 3) P13-P21 based on no statistically significant differences within each of the three age groups. Summary statistics (Table 4) for these three groups demonstrate the increase in soma size (ECD and Major Diameter) while very little change, if any in shape (Aspect Ratio and Form Factor). There was a 21\% increase in ECD from P1-P5 to P7-P12 and a 19\% increase from P7-P12 to P13-P21. No statistically significant difference was seen for aspect ratio between any of the age groups. Although the form factor was significantly different between age groups, there was a $14 \%$ increase from $\mathrm{P} 1-\mathrm{P} 5$ to $\mathrm{P} 13-\mathrm{P} 21$. Testing for normality and unimodality the ECD showed non-normality (Table 3) for two of the three groups, although unimodality was demonstrated for all three. For all three groups a mixture of two normal distributions was found to be a good-fit for the data (Tables 5-7, Figure 10). The two distributions demonstrated changes in mean values and the proportion of each distribution (Figure 10). Figure 10A shows the grouped cumulative distributions for ECD with their corresponding normal distributions. Fitting the ECD to the sum of two weighted normal distributions showed that both normal distributions changed at each stage (Figure 10B-D and Tables 5-7). The small right-skew at P1-P5 seems to be a result of the second normal distribution (Figure 10B) with an increasing tail at P7-P12 (Figure 10C) and P13-P21 (Figure 10D). Since we saw no significant differences in aspect ratio across these groups, all of the ages were pooled for the mixture model in which two normal distributions $($ distribution 1: proportion $=0.844$, mean $=1.62$, std $=0.524$, and 
distribution 2: proportion $=0.156$, mean $=2.70$, std $=0.87$ Chi-Square $\left(\chi^{2}\right)=0.391, p=$ 0.822) were found to fit the data. After subdividing the XII MNs based on their anatomical location into dorsal-ventral and lateral-medial quadrants for comparison of topographical differences, we found only the aspect ratio and form factors were statistically $(p<0.05)$ different for ages P1-P5. The aspect ratio for the dorsal-lateral region was approximately $36.5 \%$ higher than the dorsal-medial region. Although the form factor was significantly different, form factor for the dorsal-medial region was 0.6 while the dorsal-lateral region was 0.5 . No statistically significant topographical differences were found for ECD or major diameter for P1-P5 group or for any parameter in the P7P12 or P13-P21 groups. The rostral-caudal extent of XII was divided into thirds (rostral, middle, or caudal). At P1-P5 the ECD for the caudal region was $16 \%$ higher than for the rostral region and at P13-P21 the aspect ratio for the caudal region was about $17 \%$ higher than for the rostral region. Again, no differences were found for any other parameter along the rostral-caudal axis for any of the three groups.

\section{Extent and Complexity of the Dendritic Tree Increases with Age}

The P3 XII MNs had shorter dendrites with fewer branches than P10 or P12 neurons (Figure 3). The average Sholl profiles (Figure 11) demonstrated changes in dendrite morphology as changes in shape and size of the profile. At P3, 28.8\% of neurons had dendrites with longer radii of intersection (distance from soma $>150 \mu \mathrm{m}$ ) while about $10 \%$ had dendrites with intersections extending to $\geq 200 \mu \mathrm{m}$ from the soma. In contrast, at P5 and P7 about $22 \%$ of the neurons extended to $\geq 200 \mu \mathrm{m}$ from the soma. At P10 and P12 the Sholl profiles showed 5\% to $10 \%$ of neurons having dendrites with intersections 
300 to $400 \mu \mathrm{m}$ from the soma, while at P13, 23.5\% of neurons had intersections out 300 to $500 \mu \mathrm{m}$ from the soma. For P17 and P21, 27.3\% and 35.5\% of the neurons, respectively, extended 300 to $500 \mu \mathrm{m}$ from the soma. Figure 12 shows representative images of dendrite morphology from MNs (upper panels) and their morphometric values (lower panels) at P3, P10, and P21. The size and complexity of the dendritic tree is reflected by various morphometric parameters, such as the maximum number of intersections, the radius at which the maximum number of intersections occur, and the enclosing radius. Although some XII MNs may seem near symmetrical in the arrangement of the dendritic trees extending from the soma (Figure 12, P3 and P21), the total dendritic length and branching of the primary branches extending from the soma was observed to be asymmetrical for most of the neurons (Figure 12, P10 and P21).

Figure 13 shows the cumulative distributions for the maximum number of intersections (A), the radius at which the maximum number of intersections occur (B), ramification index (C), and enclosing radii (D). The maximum number of intersections (Figure 13A) and the enclosing radius (Figure 13D) demonstrated a separation between the ages with three stages defined as (1) P1, P3, and P5; (2) P7, P10, and P12; and (3) $\mathrm{P} 13, \mathrm{P} 17$, and P21. The radius at which the maximum number of intersections occur (Figure 11B) and the ramification index (Figure 13C) did not show a statistical difference between the ages. For the maximum number of intersections, radius at which the maximum number of intersections occurred, ramification index, and enclosing radius (Figure 13D) there were grouping of age ranges seen in the distributions as was seen for ECD and major diameter (Figure 8). 

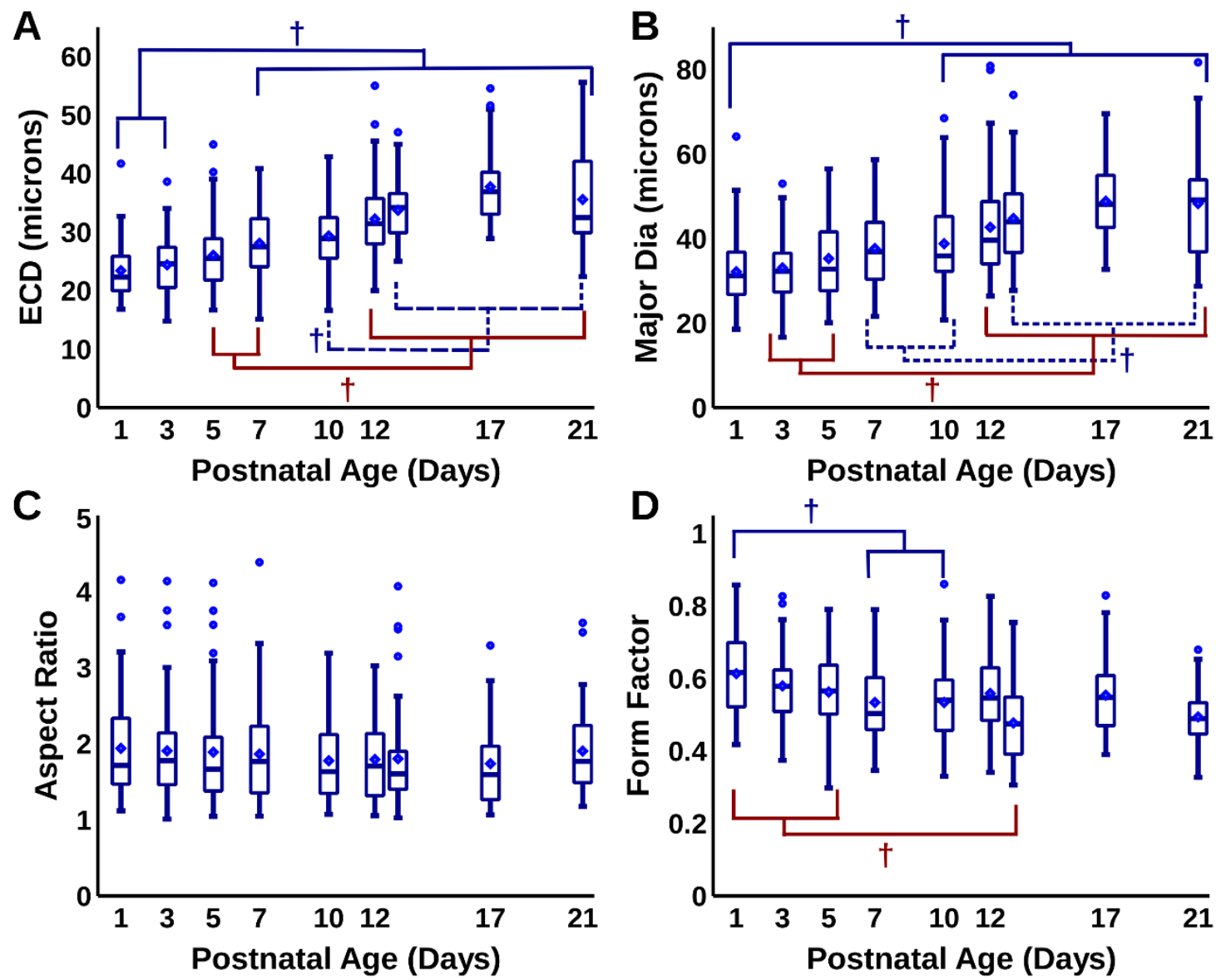

Figure 9. Box plots of the neuron soma 2D morphometrics at each postnatal age. The number of neurons for each age is found in Table 2. Shown are the a) equivalent circular diameter (ECD), b) major diameter, c) aspect ratio (AR), and d) form factor. The horizontal lines ( - ) in the boxes are the median values, the diamonds $(\bullet)$ represent the mean values, the boxes and whiskers indicate the quartiles, and the circles $(\bullet)$ are points greater than 1.5 times the interquartile range (IQR). Non-parametric Kruskal-Wallis with pairwise non-parametric multiple comparison were performed, statistical significance was determined as $\mathrm{p}<0.05$, and denoted with an $\uparrow$. 
Table 3. Shapiro-Wilk normality test results for soma morphology. Shown for each parameter and age are the $\mathrm{W}$-statistic and the corresponding $\mathrm{p}$-value. The three bottom rows are for the three groups. Cells in bold are significantly different at the $p<0.01$ level.

\begin{tabular}{llrlrlllll}
\hline \multirow{2}{*}{$\begin{array}{c}\text { Age } \\
\text { (days) }\end{array}$} & \multicolumn{2}{c}{ ECD $(\boldsymbol{\mu m})$} & \multicolumn{3}{c}{ Major Dia $(\boldsymbol{\mu m})$} & \multicolumn{2}{c}{ Aspect Ratio } & \multicolumn{2}{c}{ Form Factor } \\
\cline { 2 - 10 } & $\mathbf{W}$ & $\mathbf{p}$-value & \multicolumn{1}{c}{ W } & p-value & W & p-value & W & p-value \\
\hline P1 & 0.906 & $\mathbf{0 . 0 0 1}$ & 0.923 & $\mathbf{0 . 0 0 4}$ & 0.892 & $<\mathbf{0 . 0 0 1}$ & 0.964 & 0.145 \\
P3 & 0.980 & 0.455 & 0.971 & 0.183 & 0.883 & $<\mathbf{0 . 0 0 1}$ & 0.980 & 0.438 \\
P5 & 0.911 & $\mathbf{0 . 0 0 2}$ & 0.941 & 0.019 & 0.859 & $<\mathbf{0 . 0 0 1}$ & 0.991 & 0.966 \\
P7 & 0.983 & 0.554 & 0.973 & 0.200 & 0.909 & $<\mathbf{0 . 0 0 1}$ & 0.943 & $\mathbf{0 . 0 0 6}$ \\
P10 & 0.986 & 0.686 & 0.966 & 0.079 & 0.916 & $<\mathbf{0 . 0 0 1}$ & 0.974 & 0.202 \\
P12 & 0.944 & $\mathbf{0 . 0 1 0}$ & 0.896 & $<\mathbf{0 . 0 0 1}$ & 0.916 & $<\mathbf{0 . 0 0 1}$ & 0.985 & 0.669 \\
P13 & 0.980 & 0.528 & 0.956 & 0.055 & 0.799 & $<\mathbf{0 . 0 0 1}$ & 0.972 & 0.262 \\
P17 & 0.926 & $\mathbf{0 . 0 0 7}$ & 0.980 & 0.647 & 0.918 & $\mathbf{0 . 0 0 4}$ & 0.948 & 0.047 \\
P21 & 0.941 & 0.089 & 0.940 & 0.081 & 0.899 & $\mathbf{0 . 0 0 7}$ & 0.973 & 0.593 \\
\hline P1-P5 & 0.948 & $<\mathbf{0 . 0 0 1}$ & 0.958 & $<\mathbf{0 . 0 0 1}$ & 0.881 & $<\mathbf{0 . 0 0 1}$ & 0.992 & 0.544 \\
P7-P12 & 0.980 & 0.010 & 0.950 & $<\mathbf{0 . 0 0 1}$ & 0.922 & $<\mathbf{0 . 0 0 1}$ & 0.977 & $\mathbf{0 . 0 0 7}$ \\
P13-P21 & 0.959 & $\mathbf{0 . 0 0 1}$ & 0.969 & $\mathbf{0 . 0 0 5}$ & 0.873 & $<\mathbf{0 . 0 0 1}$ & 0.975 & 0.019 \\
\hline
\end{tabular}


Table 4. Soma morphology summary statistics (mean \pm std) for the three age groups.

\begin{tabular}{lccc}
\hline \multicolumn{1}{c}{ Parameter } & P1-P5 & P7-P12 & P13-P21 \\
\hline Area $\left(\mu \mathbf{m}^{\mathbf{3}}\right)$ & $497 \pm 230$ & $729 \pm 314$ & $1028 \pm 406$ \\
Perimeter $(\mu \mathrm{m})$ & $102 \pm 25$ & $129 \pm 30$ & $159 \pm 35$ \\
Major Diameter $(\boldsymbol{\mu m})$ & $33.5 \pm 8.9$ & $39.6 \pm 10.4$ & $47.0 \pm 10.7$ \\
Minor Diameter $(\boldsymbol{\mu m})$ & $18.5 \pm 4.9$ & $23.0 \pm 5.8$ & $27.5 \pm 7.0$ \\
ECD $(\boldsymbol{\mu m})$ & $24.6 \pm 5.3$ & $29.8 \pm 6.2$ & $35.6 \pm 6.7$ \\
Aspect Ratio & $1.91 \pm 0.69$ & $1.81 \pm 0.58$ & $1.80 \pm 0.60$ \\
Form Factor & $0.58 \pm 0.11$ & $0.54 \pm 0.10$ & $0.51 \pm 0.10$ \\
\hline
\end{tabular}


Table 5. Results of fitting two normal distributions to various parameters for P1-P5 (P1, P3, \& P5). Shown are the proportion (prop) or weight for that distribution, the mean, the $\mathrm{SD}$, and the $p$-value for the mixture distribution model.

\begin{tabular}{lrrrrrrrr}
\hline \multirow{2}{*}{ Parameters } & \multicolumn{9}{c}{ Normal Distribution 1 } & \multicolumn{2}{c}{ Normal Distribution 2 } & \multicolumn{2}{c}{$\begin{array}{c}\text { Chi- } \\
\text { Square }\end{array}$} & p-value \\
\cline { 2 - 8 } & \multicolumn{1}{c}{ prop } & \multicolumn{1}{c}{ mean } & \multicolumn{1}{c}{ std } & prop & mean & std & & \\
\hline ECD $(\boldsymbol{\mu m})$ & 0.971 & 24.28 & 4.59 & 0.029 & 43.74 & 8.26 & 3.77 & 0.152 \\
Maj Dia $(\boldsymbol{\mu m})$ & 0.705 & 29.60 & 5.52 & 0.295 & 43.18 & 8.05 & 2.05 & 0.842 \\
FF & N/A & 0.59 & 0.11 & N/A & N/A & N/A & N/A & 0.544 \\
Max Int & 0.417 & 4.17 & 1.13 & 0.583 & 7.49 & 2.03 & 2.77 & 0.837 \\
Max Int R $(\boldsymbol{\mu m})$ & 0.412 & 20.47 & 7.23 & 0.588 & 43.01 & 15.19 & 0.42 & 0.812 \\
Ram Index & 0.768 & 1.19 & 0.41 & 0.232 & 2.33 & 0.80 & 0.72 & 0.697 \\
Encl Rad $(\boldsymbol{\mu m})$ & 0.822 & 108.50 & 32.16 & 0.178 & 227.60 & 67.46 & 16.31 & 0.130 \\
\hline
\end{tabular}


Table 6. Results of fitting two normal distributions to various parameters for P7-P12 (P7, $\mathrm{P} 10, \& \mathrm{P} 12)$. Shown are the proportion (prop) or weight for that distribution, the mean, the SD, and the $p$-value for the mixture distribution model.

\begin{tabular}{|c|c|c|c|c|c|c|c|c|}
\hline \multirow[t]{2}{*}{ Parameters } & \multicolumn{3}{|c|}{ Normal Distribution 1} & \multicolumn{3}{|c|}{ Normal Distribution 2} & \multirow{2}{*}{$\begin{array}{c}\text { Chi- } \\
\text { Square }\end{array}$} & \multirow{2}{*}{ p-value } \\
\hline & prop & mean & std & prop & mean & std & & \\
\hline $\operatorname{ECD}(\mu \mathrm{m})$ & 0.819 & 28.40 & 4.73 & 0.181 & 37.27 & 6.20 & 0.78 & 0.855 \\
\hline Maj Dia $(\mu \mathrm{m})$ & 0.293 & 32.94 & 4.33 & 0.707 & 41.33 & 10.38 & 3.65 & 0.456 \\
\hline FF & 0.626 & 0.49 & 0.07 & 0.374 & 0.63 & 0.09 & 14.05 & 0.900 \\
\hline Max Int & 0.822 & 7.64 & 2.18 & 0.178 & 13.08 & 2.63 & 1.20 & 0.752 \\
\hline Max Int R $(\mu \mathrm{m})$ & 0.545 & 35.24 & 17.68 & 0.455 & 66.01 & 33.12 & 3.19 & 0.867 \\
\hline Ram Index & 0.754 & 1.45 & 0.48 & 0.246 & 2.44 & 0.80 & 11.44 & 0.324 \\
\hline $\operatorname{Encl} \operatorname{Rad}(\mu \mathrm{m})$ & 0.899 & 177.60 & 51.45 & 0.101 & 328.30 & 95.13 & 19.93 & 0.462 \\
\hline
\end{tabular}


Table 7. Results of fitting two normal distributions to various parameters for P13-P21 (P13, P17, \& P21). Shown are the proportion (prop) or weight for that distribution, the mean, the $\mathrm{SD}$, and the $p$-value for the mixture distribution model.

\begin{tabular}{|c|c|c|c|c|c|c|c|c|}
\hline \multirow[t]{2}{*}{ Parameters } & \multicolumn{3}{|c|}{ Normal Distribution 1} & \multicolumn{3}{|c|}{ Normal Distribution 2} & \multirow{2}{*}{$\begin{array}{c}\text { Chi- } \\
\text { Square }\end{array}$} & \multirow{2}{*}{ p-value } \\
\hline & prop & mean & std & prop & mean & std & & \\
\hline $\mathbf{E C D}(\mu \mathrm{m})$ & 0.776 & 33.27 & 4.75 & 0.224 & 43.97 & 6.28 & 1.67 & 0.643 \\
\hline Maj Dia $(\mu \mathrm{m})$ & 0.108 & 35.17 & 0.84 & 0.892 & 47.87 & 9.97 & 14.10 & 0.723 \\
\hline FF & 0.791 & 0.47 & 0.09 & 0.204 & 0.62 & 0.11 & 5.14 & 0.526 \\
\hline Max Int & 0.785 & 9.59 & 2.77 & 0.215 & 15.15 & 4.38 & 19.01 & 0.165 \\
\hline Max Int R ( $\mu \mathrm{m})$ & 0.898 & 63.05 & 25.95 & 0.102 & 128.07 & 52.70 & 13.21 & 0.432 \\
\hline Ram Index & 0.723 & 1.77 & 0.50 & 0.277 & 3.00 & 0.85 & 10.80 & 0.701 \\
\hline Encl Rad $(\mu \mathrm{m})$ & 0.363 & 185.10 & 35.19 & 0.637 & 282.60 & 103.23 & 6.97 & 0.324 \\
\hline
\end{tabular}



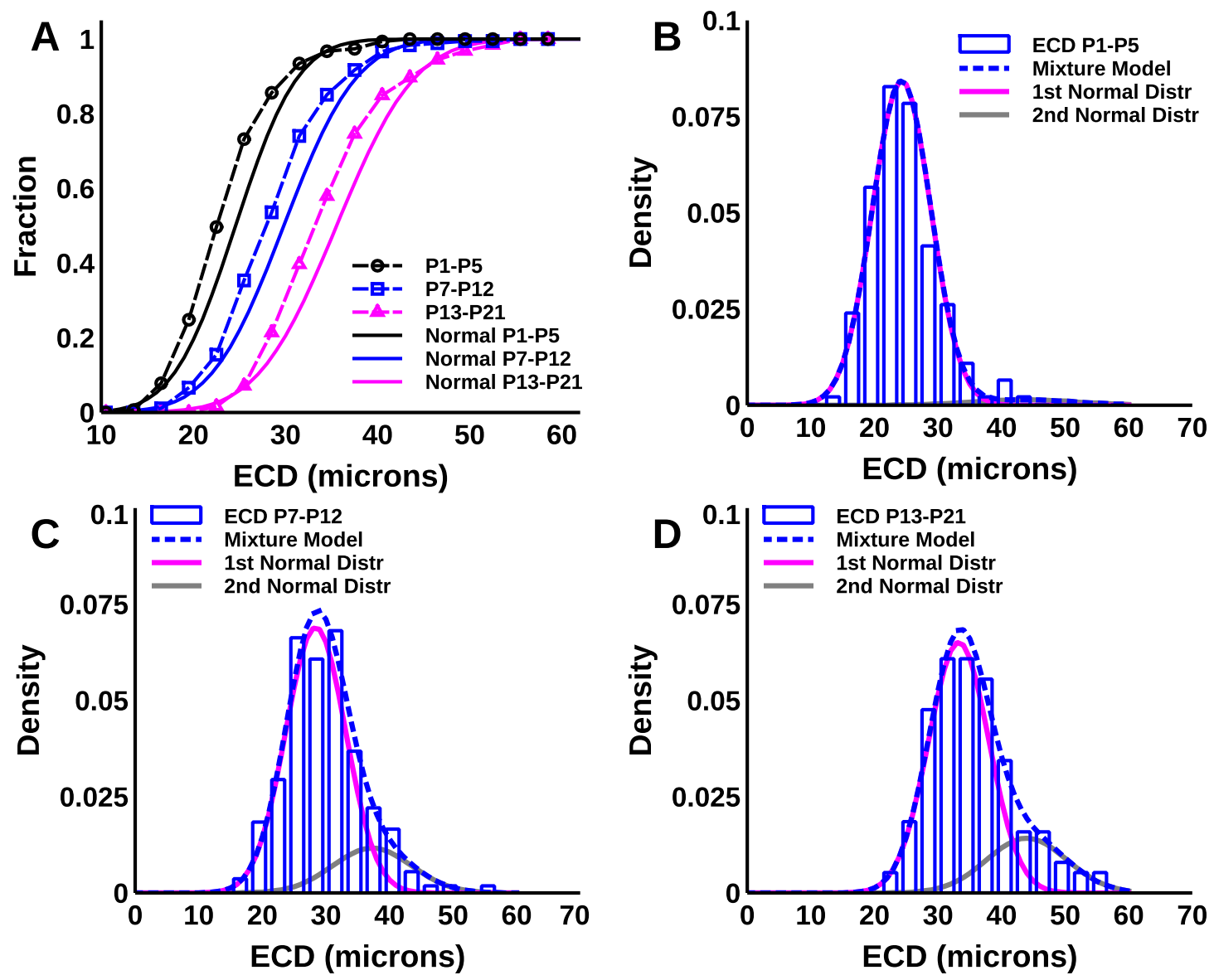

Figure 10. Pooled distributions for the ECD showing the finite mixture distribution models. a) Cumulative and corresponding normal distributions for ECD at P1-P5 (P1, P3, \& P5), P7-P12 (P7, P10, \& P12), and P13-P21 (P13, P17, \& P21). b-d) Histograms for the ECD and mixture distribution models showing the two normal distributions for b) P1-P5, c) P7-P12, and d) P13-P21. The parameters for the two normal distributions are given in Tables 5, 6, and 7 . 

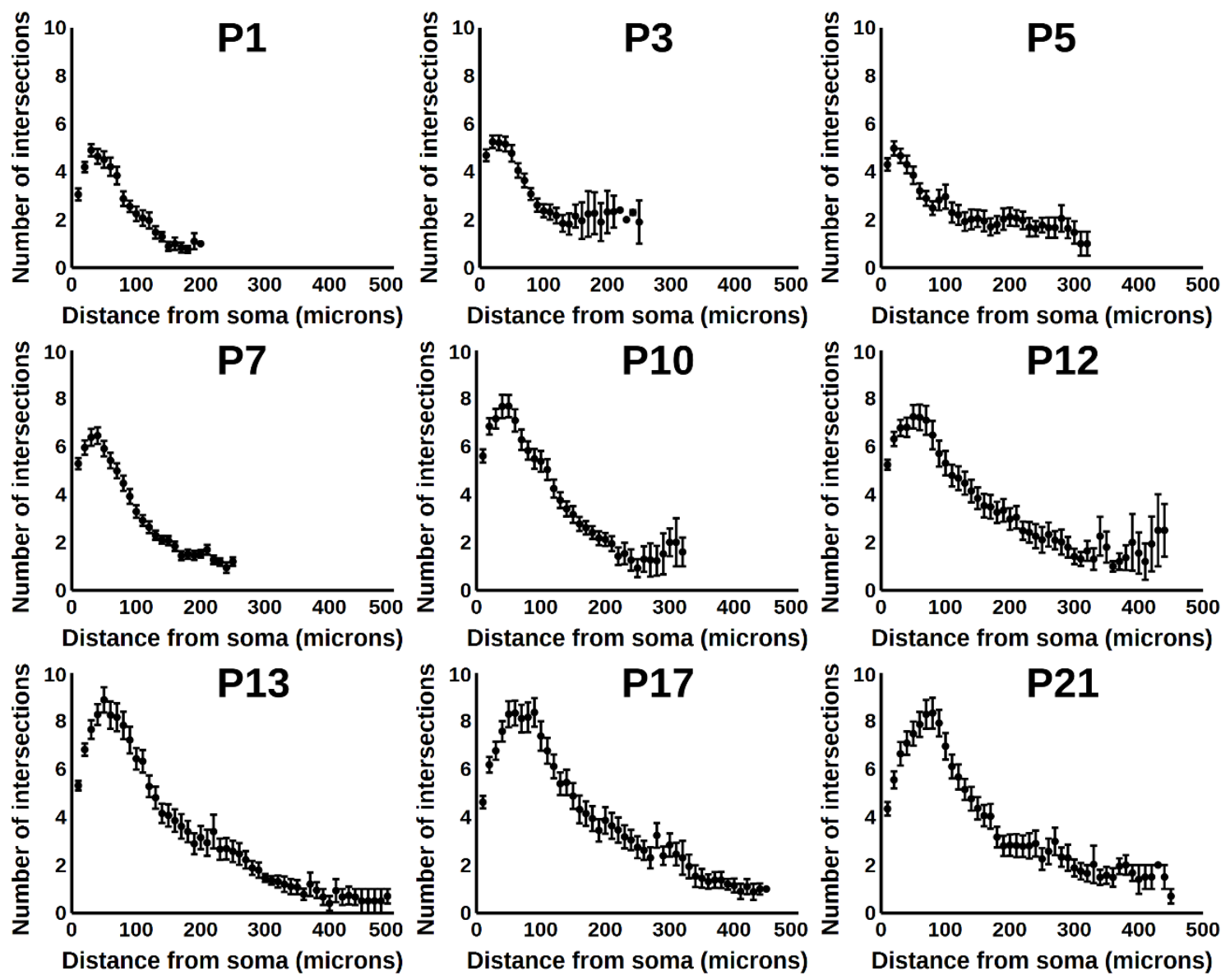

Figure 11. Sholl profiles for the nine postnatal ages. Each data point is the mean value with the error bars representing the standard error. The number of neurons for each graph is shown in Table 2, however the number of neurons per data point may decrease with increasing distance due to some neurons having a shorter enclosing radius as compared to other neurons. For some profiles (P3, P10, and P12) the variance at the larger distances are due to only a few neurons having dendrites extending to that distance. 

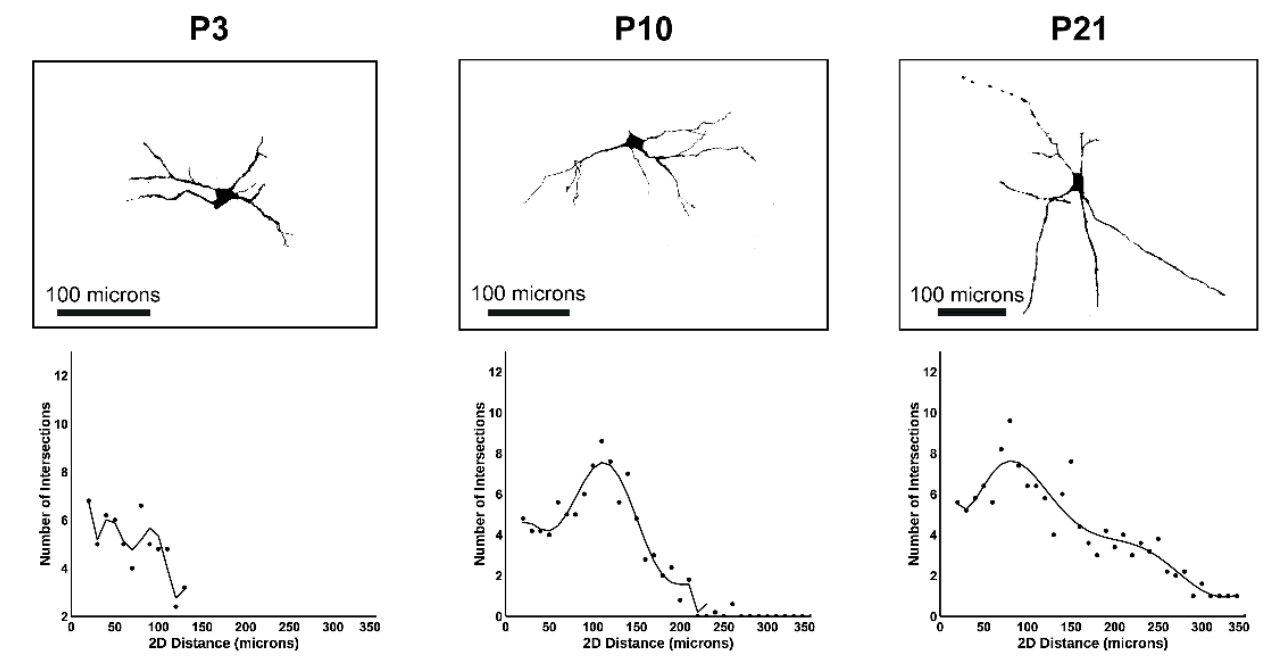

Max Int $=6.8 ;$ Max Int Rad $=20 \mathrm{~mm}$ Ram Index = 1.00; Encl Rad $=130 \mathrm{~mm}$

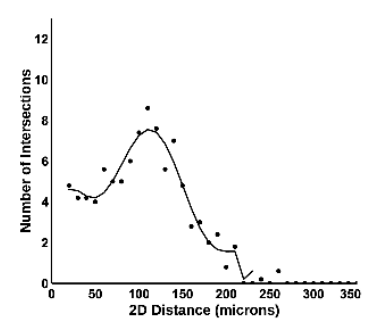

Max Int $=8.6 ;$ Max Int Rad $=110 \mathrm{~mm}$ Ram Index = 1.79; Encl Rad $=210 \mathrm{~mm}$

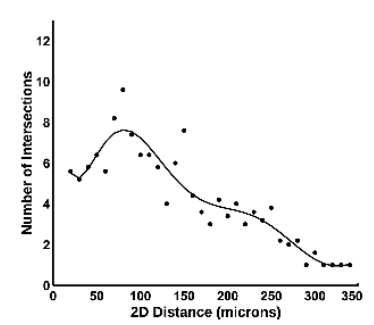

Max Int = 9.6; Max Int Rad $=80 \mathrm{~mm}$ Ram Index = 1.71; Encl Rad $=340 \mathrm{~mm}$

Figure 12. Representative threshold images of isolated XII MNs from P3, P10, and P21 with their respective Sholl profile and values for the maximum number of intersections (Max Int), radius at which the maximum number of intersections occur (Max Int Rad), ramification index (Ram Index), and enclosing radius (Encl Rad). For the arrows indicate the neurons which correspond to the Sholl profile. 

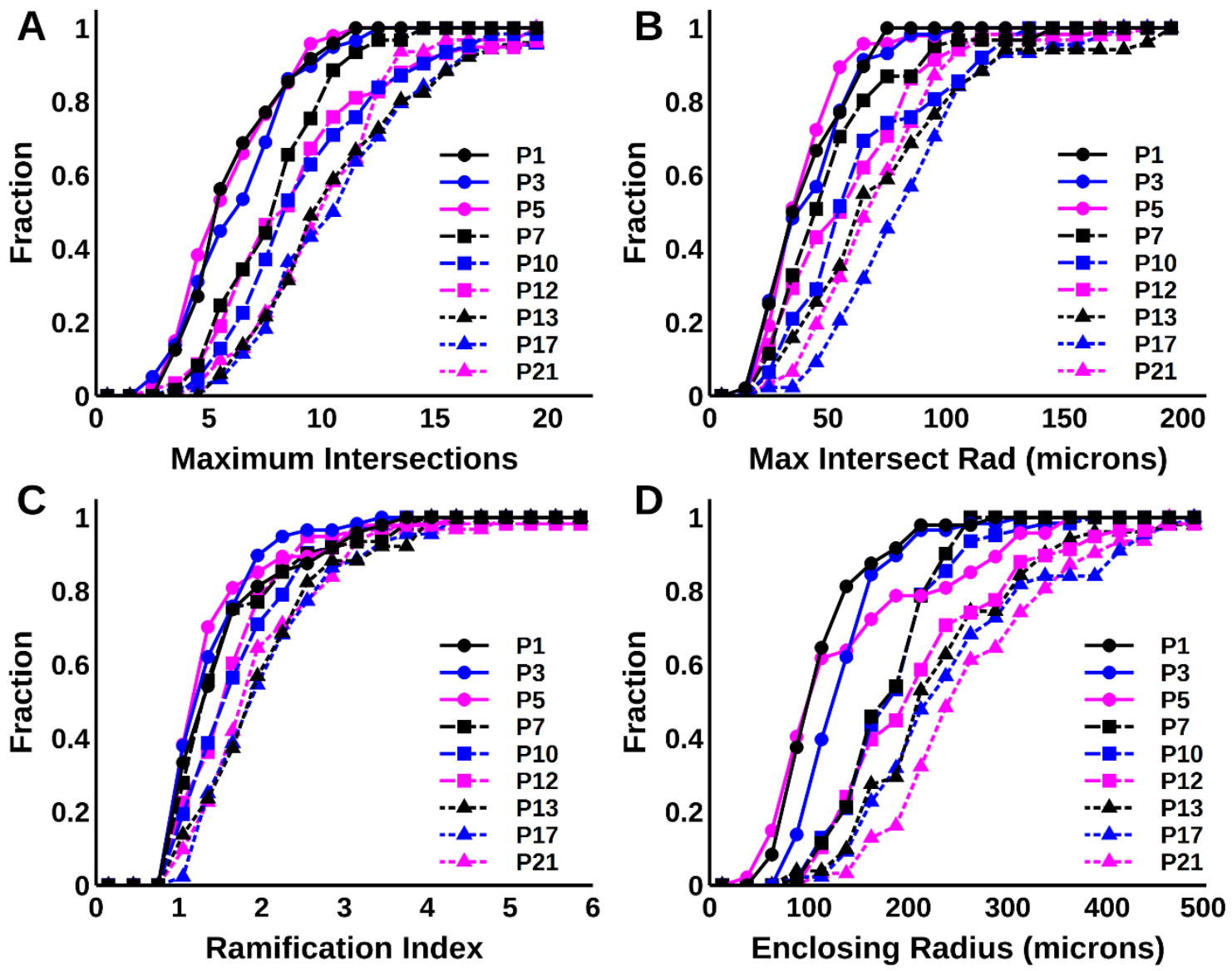

Figure 13. Cumulative distributions for A) maximum number of intersections, B) radius at which maximum number of intersections occurred, C) ramification index, and D) enclosing radius at the nine postnatal ages (P1, P3, P5, P7, P10, P12, P13, P17, and P21). The $\mathrm{y}$-axis shows the cumulative fraction such that 0.2 is $20 \%, 0.6$ is $60 \%$, and 1 is $100 \%$. Results from the Shapiro-Wilks test for normality are in Table 8 and the results of the Hartigan's dip test for unimodality are shown in Table 9. 
The maximum number of intersections (number of dendrites intersecting a concentric circle) at P10-P21 were significantly higher than P1-P5 (Figure 14A) by 50\% to $90 \%$. Similarly, the maximum intersection radius (radial distance from soma at which the maximum number of intersections occur) was age-dependent with a $30 \%$ or greater increase at P10-P21 (Figure 12B). The maximum intersection radius at P13, P17, and P21 were also significantly higher by about $40 \%$ or greater than P7 (Figure 14B). For the ramification index P13 and P17 were significantly higher by approximately $40 \%$ than P1-P7 (Figure 14C) which again may indicate that the increase in maximum number of intersections for P7-P12 may be mainly due to the increase in the number of primary branches, but not at P13 and P17 (Figures 14A and 14C). We did observe an increase in the number of primary branches from P1 to P21 with a statistically significant $25 \%$ to 50\% increase for P7-P17 compared with P1. However, the number of primary branches increased gradually leveling off from P13-P21. For the enclosing radius P7-P21 were significantly increased by $50 \%$ or more from $\mathrm{P} 1$ and $\mathrm{P} 3$ while $\mathrm{P} 13$ through $\mathrm{P} 21$ were significantly increased by $40 \%$ compared with P7 and P10 (Figure 14D). The majority of the parameters showed significant increases starting between P10 and P13 and a few cases at P7. In the ventral-lateral region the enclosing radius for the P1-P5 group was found to be significantly $(p<0.05)$ lower from the other three by approximately $29 \%$, but there were no differences found for the other age groups. The ramification index for the dorsal-lateral region was significantly higher (approximately 33\%) when compared with the ventral-medial region for the P13-P21 group. In the rostral-caudal direction ramification index for the P7-P12 group was significantly lower by $21.2 \%$ in the caudal 
region as compared with the rostral region. No other differences were found for any parameter or age related to topographical location.

\section{Dendrite Morphology: Normality and Unimodality}

The Shapiro-Wilk test showed significant $(p<0.01)$ deviation from normality for the $\mathrm{P} 1, \mathrm{P} 10$, and $\mathrm{P} 12$ groups for maximum number of intersections while the radius at which the maximum number of intersections occurred was significantly different from the normal distribution at P1 through P13 (Table 8). The ramification index was significantly different from a normal distribution for all ages (Table 8). For the enclosing radius at $\mathrm{P} 1, \mathrm{P} 3, \mathrm{P} 5, \mathrm{P} 12, \mathrm{P} 13$ and $\mathrm{P} 17$ the data is significantly different from a normal distribution, but not at P7, P10, or P21. Applying the Hartigan's dip test for unimodality to the radius at which the maximum number of intersections occurred revealed a distribution that was significantly different from a unimodal distribution at P1-P12, but not at P13, P17, or P21 (Table 9). For all ages, the other three morphometric parameters were not significantly different from a unimodal distribution (Table 9). In summary, depending on the specific parameter of interest, XII MN dendrite morphology at some postnatal ages exhibited a non-normal distribution while at other ages they were normally distributed.

Figures 15A and 15B show examples of the cumulative distributions for data which were normally/non-normally distributed (Figure 15A) or were unimodally/nonunimodally (bimodal or multimodal) distributed (Figure 15B). Curve fitting the data for the maximum number of intersections for P10 yielded a log-normal distribution (Figure $15 \mathrm{C}$, dashed line) which seems to follow the same form as the data, or a mixture distribution model of two normal distributions (Figure 15C, solid line) both 
demonstrating a good fit to the data. For the maximum number of intersections radius for P10 a mixture distribution of two normal distributions (Figure 15D) was found to fit the data best. To provide sufficient numbers for fitting finite mixture distribution model the data was again pooled into three groups (P1-P5, P7-P12, and P13-P21). Summary statistics (Table 10) for the three groups showed an increase in number of primary branches from P1-P5 to P7-P12 with no significant change between P7-P12 and P13P21. However, the maximum number of intersections significantly increased from P1-P5 to P7-P12 and from P7-P12 to P13-P21. Testing for normality and unimodality for the dendritic morphology of the pooled data showed that most of the data was non-normally distributed (Table 8), but most were unimodally distributed except for the radius of the maximum number of intersections and enclosing radius (Table 11). Figure 14A shows the cumulative distributions of the maximum intersection radii for the three groups with their corresponding normal distribution and demonstrates the non-normality of the data. Fitting the maximum intersection radius to the sum of two weighted normal distributions showed that at P1-P5 two distributions of comparable weights (Figure 16B and Tables 5-7) with shifts in both distributions at P7-P12 (Figure 14C) and one dominant distribution at P13P21 (Figure 16D). Figure 15 illustrates three sets of images in which two different neurons in proximity of each other have very different Sholl profiles. In some sections two neurons can be found which represent near minimum and maximum value (Figure 17). Again, while a few XII MNs might exhibit some symmetry in total dendritic length and branching of primary branches extending from the soma (Figure 17, P3 left side of image), most XII MNs demonstrated asymmetrical total dendritic length and branching. 

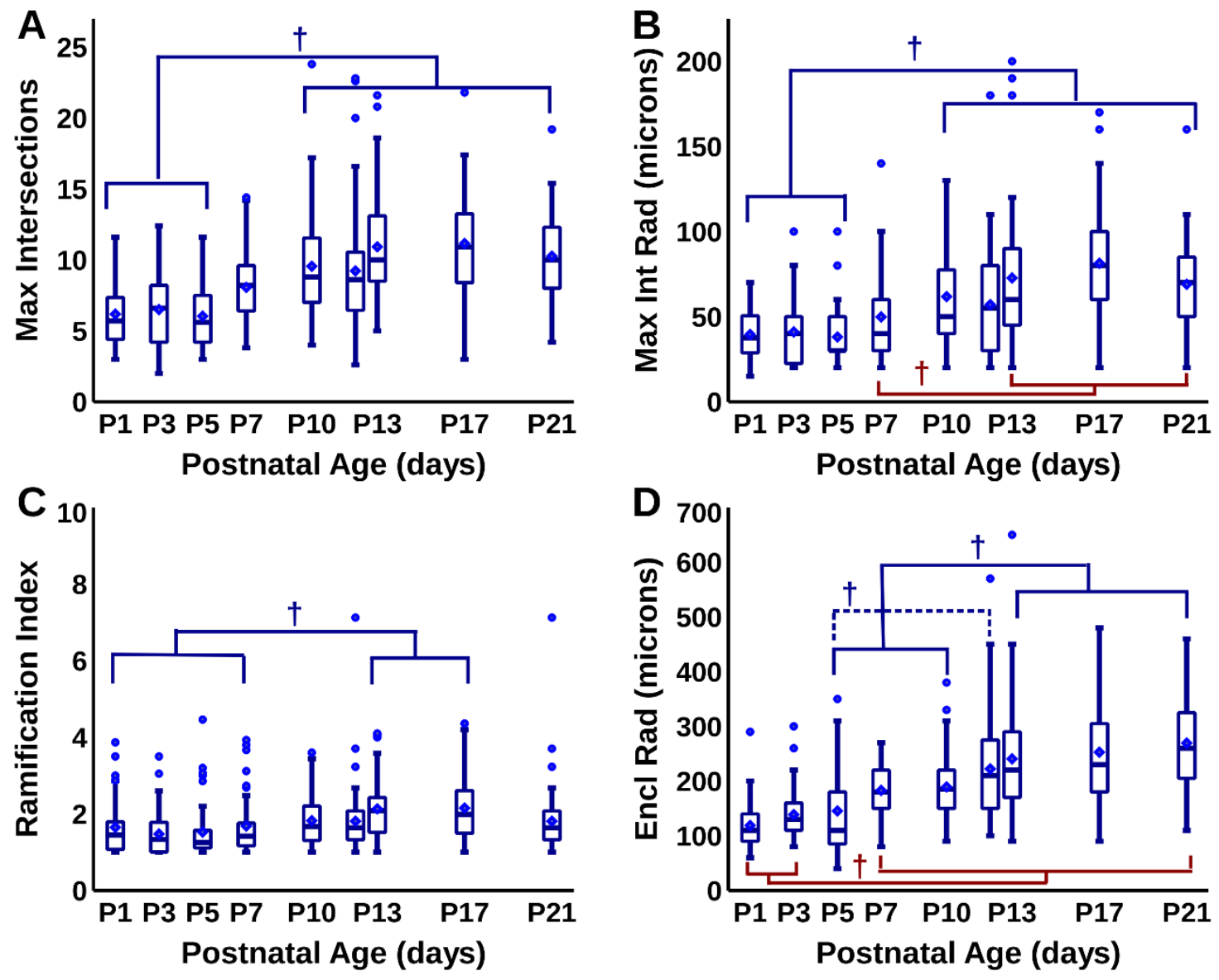

Figure 14. Box plots of the 2D Sholl analysis morphometrics measured at each of the nine postnatal ages. The number of neurons for each age is found in Table 2. Shown are the a) maximum number of intersections, b) radius at which the maximum number of intersections occurred, c) ramification index, and d) enclosing radius. The horizontal lines in the boxes are the median values while the diamonds are the mean values and the circles are points greater than 1.5 times the IQR. For each parameter Non-parametric KruskalWallis with pairwise non-parametric multiple comparison were performed to compare the different postnatal ages. Statistical significance was determined as $p<0.05$ and denoted with an $\dagger$. 
Table 8. Shapiro-Wilk normality test results for dendrite morphology. Shown for each parameter and age are the $\mathrm{W}$-statistic and the corresponding $\mathrm{p}$-value. The three bottom rows are for the three groups. Cells in bold are significantly different at the $p<0.01$ level.

\begin{tabular}{lcrrrrrrrr}
\hline \multirow{2}{*}{$\begin{array}{c}\text { Age } \\
\text { (days) }\end{array}$} & \multicolumn{2}{c}{$\begin{array}{c}\text { Max } \\
\text { Intersections }\end{array}$} & \multicolumn{2}{c}{$\begin{array}{c}\text { Max Intersect } \\
\text { Rad }\end{array}$} & \multicolumn{2}{c}{$\begin{array}{c}\text { Ramification } \\
\text { Index }\end{array}$} & \multicolumn{2}{c}{$\begin{array}{c}\text { Enclosing } \\
\text { Radius }\end{array}$} \\
\cline { 2 - 10 } W & p-value & W & p-value & W & p-value & W & p-value \\
\hline P1 & 0.928 & $\mathbf{0 . 0 0 6}$ & 0.904 & $\mathbf{0 . 0 0 1}$ & 0.829 & $<\mathbf{0 . 0 0 1}$ & 0.868 & $<\mathbf{0 . 0 0 1}$ \\
P3 & 0.975 & 0.266 & 0.890 & $<\mathbf{0 . 0 0 1}$ & 0.829 & $<\mathbf{0 . 0 0 1}$ & 0.908 & $<\mathbf{0 . 0 0 1}$ \\
P5 & 0.952 & 0.049 & 0.847 & $<\mathbf{0 . 0 0 1}$ & 0.706 & $<\mathbf{0 . 0 0 1}$ & 0.846 & $<\mathbf{0 . 0 0 1}$ \\
P7 & 0.974 & 0.219 & 0.848 & $<\mathbf{0 . 0 0 1}$ & 0.793 & $<\mathbf{0 . 0 0 1}$ & 0.975 & 0.253 \\
P10 & 0.924 & $\mathbf{0 . 0 0 1}$ & 0.902 & $<\mathbf{0 . 0 0 1}$ & 0.926 & $\mathbf{0 . 0 0 1}$ & 0.962 & 0.054 \\
P12 & 0.892 & $<\mathbf{0 . 0 0 1}$ & 0.896 & $<\mathbf{0 . 0 0 1}$ & 0.669 & $<\mathbf{0 . 0 0 1}$ & 0.901 & $<\mathbf{0 . 0 0 1}$ \\
P13 & 0.943 & 0.016 & 0.884 & $<\mathbf{0 . 0 0 1}$ & 0.917 & $\mathbf{0 . 0 0 2}$ & 0.888 & $<\mathbf{0 . 0 0 1}$ \\
P17 & 0.960 & 0.125 & 0.954 & 0.076 & 0.912 & $\mathbf{0 . 0 0 3}$ & 0.924 & $\mathbf{0 . 0 0 7}$ \\
P21 & 0.976 & 0.701 & 0.936 & 0.065 & 0.902 & $\mathbf{0 . 0 0 8}$ & 0.965 & $\mathbf{0 . 3 8 4}$ \\
\hline P1-P5 & 0.966 & $\mathbf{0 . 0 0 1}$ & 0.901 & $<\mathbf{0 . 0 0 1}$ & 0.788 & $<\mathbf{0 . 0 0 1}$ & 0.862 & $<\mathbf{0 . 0 0 1}$ \\
P7-P12 & 0.913 & $<\mathbf{0 . 0 0 1}$ & 0.906 & $<\mathbf{0 . 0 0 1}$ & 0.798 & $<\mathbf{0 . 0 0 1}$ & 0.903 & $<\mathbf{0 . 0 0 1}$ \\
P13-P21 & 0.962 & $\mathbf{0 . 0 0 1}$ & 0.922 & $<\mathbf{0 . 0 0 1}$ & 0.925 & $<\mathbf{0 . 0 0 1}$ & 0.936 & $<\mathbf{0 . 0 0 1}$ \\
\hline
\end{tabular}


Table 9. Results from Hartigans' dip test for unimodality/multimodality for dendrite morphology. Shown for each parameter and age are the D-statistic and the corresponding p-value. The three bottom rows are for the three groups. Cells in bold are significantly different at the $\mathrm{p}<0.01$ level.

\begin{tabular}{lccccccccc}
\hline \multirow{2}{*}{$\begin{array}{c}\text { Age } \\
\text { (days) }\end{array}$} & \multicolumn{2}{c}{$\begin{array}{c}\text { Max } \\
\text { Intersections }\end{array}$} & \multicolumn{2}{c}{$\begin{array}{c}\text { Max Intersect } \\
\text { Rad }\end{array}$} & \multicolumn{2}{c}{$\begin{array}{c}\text { Ramification } \\
\text { Index }\end{array}$} & \multicolumn{2}{c}{$\begin{array}{c}\text { Enclosing } \\
\text { Radius }\end{array}$} \\
\cline { 2 - 10 } & $\mathbf{D}$ & p-value & \multicolumn{1}{l}{ D } & p-value & \multicolumn{1}{c}{ D } & p-value & \multicolumn{1}{c}{ D } & p-value \\
\hline P1 & 0.049 & 0.524 & 0.104 & $<\mathbf{0 . 0 0 1}$ & 0.051 & 0.469 & 0.052 & 0.416 \\
P3 & 0.054 & 0.224 & 0.112 & $<\mathbf{0 . 0 0 1}$ & 0.044 & 0.584 & 0.060 & 0.103 \\
P5 & 0.043 & 0.787 & 0.106 & $<\mathbf{0 . 0 0 1}$ & 0.054 & 0.361 & 0.053 & 0.395 \\
P7 & 0.049 & 0.325 & 0.098 & $<\mathbf{0 . 0 0 1}$ & 0.039 & 0.727 & 0.066 & 0.042 \\
P10 & 0.028 & 0.990 & 0.089 & $\mathbf{0 . 0 0 1}$ & 0.035 & 0.868 & 0.048 & 0.340 \\
P12 & 0.065 & 0.053 & 0.078 & $\mathbf{0 . 0 0 8}$ & 0.049 & 0.388 & 0.052 & 0.284 \\
P13 & 0.039 & 0.844 & 0.054 & 0.312 & 0.034 & 0.960 & 0.049 & 0.477 \\
P17 & 0.042 & 0.835 & 0.068 & 0.107 & 0.043 & 0.824 & 0.037 & 0.950 \\
P21 & 0.050 & 0.832 & 0.065 & 0.386 & 0.037 & 0.991 & 0.048 & 0.860 \\
\hline P1-P5 & 0.033 & 0.293 & 0.111 & $<\mathbf{0 . 0 0 1}$ & 0.025 & 0.781 & 0.043 & 0.044 \\
P7-P12 & 0.026 & 0.539 & 0.083 & $<\mathbf{0 . 0 0 1}$ & 0.027 & 0.522 & 0.048 & $\mathbf{0 . 0 0 5}$ \\
P13-P21 & 0.031 & 0.571 & 0.058 & $\mathbf{0 . 0 0 3}$ & 0.026 & 0.831 & 0.036 & 0.297 \\
\hline & & & & & & & &
\end{tabular}



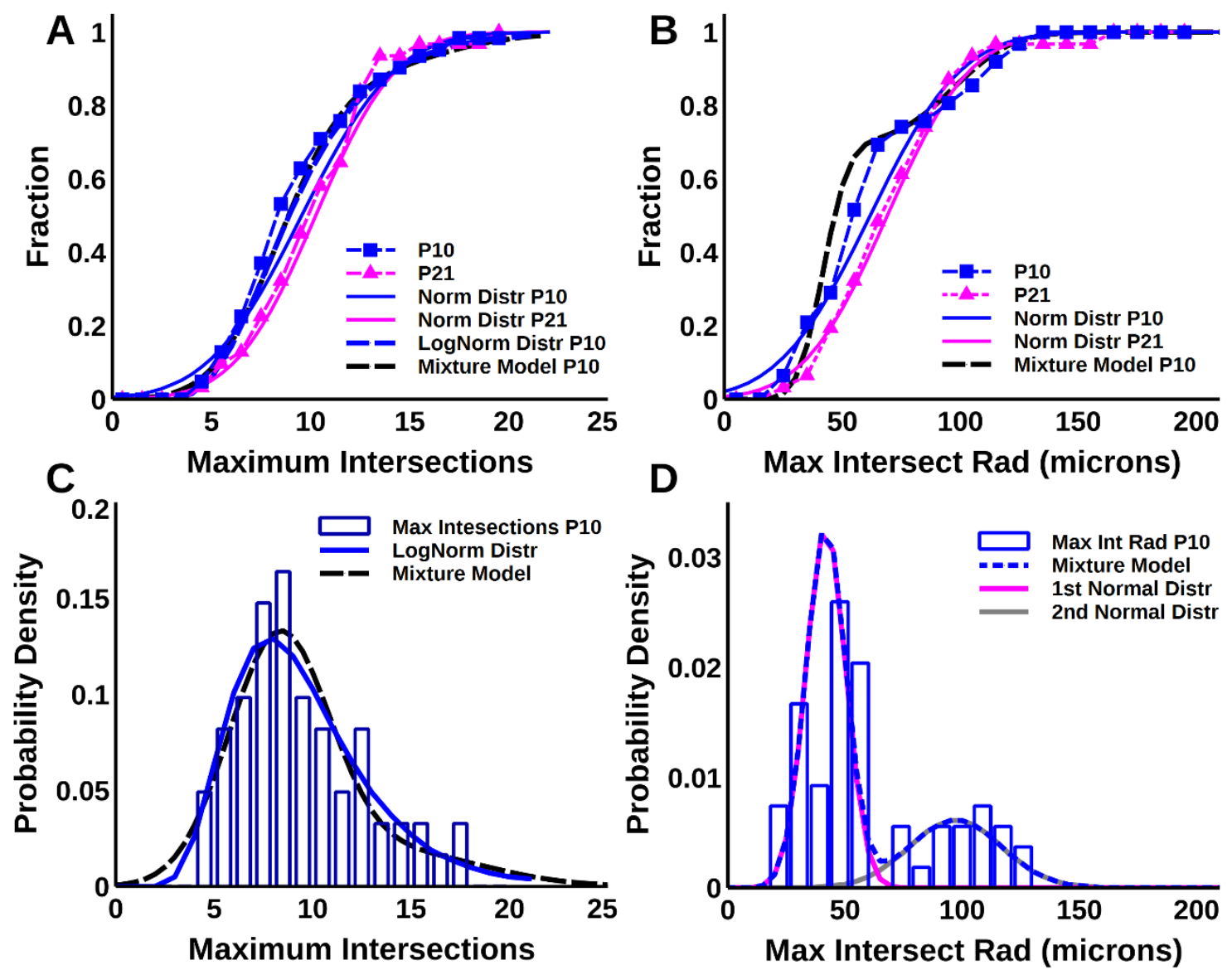

Figure 15. Distributions showing normal unimodal, non-normal unimodal, and nonnormal non-unimodal data. a) Cumulative distributions of the maximum number of intersections with the P21 data demonstrating a normal unimodal distribution as compared to the normal line while the P10 data exhibits a unimodal, but not a normal distribution illustrated by the distance of the points from the normal line. b) Cumulative distributions of the maximum intersections radius with the P21 data showing a normal and unimodal distribution as compared to the line while the P10 data demonstrates a bimodal or multimodal distribution as exhibited by the distance and pattern of the points to the reference normal line. c) Histogram of the maximum number of intersections with a lognormal distribution (log-mean $=2.19, \log -\mathrm{SD}=0.37, \mathrm{p}$-value $=0.6783$ ) and finite mixture model (normal distribution-1: weight $=0.806$, mean=8.28, $\mathrm{SD}=2.55$; distribution-2: weight=0.1941, mean=14.26.22, $\mathrm{SD}=4.40$; Chi-sq=17.305, $p$-value=0.3010). d) Histogram of the maximum number of intersections radius with the two normal distributions and the finite mixture distribution model (normal distribution-1: weight $=0.697$, mean $=41.85$, $\mathrm{SD}=8.47$; distribution-2: weight=0.303, mean=97.22, $\mathrm{SD}=19.69 ;$ Chi-sq=0.333, $p$ value $=0.5636$ ) . 
Table 10. Dendrite morphology summary statistics (mean \pm std) for the three age groups.

\begin{tabular}{lccc}
\hline \multicolumn{1}{c}{ Parameter } & P1-P5 & P7-P12 & P13-P21 \\
\hline Number of Primary Branches & $4.38 \pm 1.72$ & $5.37 \pm 1.88$ & $5.33 \pm 1.42$ \\
Max Number of Intersections & $6.26 \pm 2.31$ & $8.94 \pm 3.60$ & $10.84 \pm 3.78$ \\
Radius at Max Intersections $(\boldsymbol{\mu m})$ & $39.7 \pm 17.6$ & $56.2 \pm 26.4$ & $74.84 \pm 34.7$ \\
Ramification index & $1.54 \pm 0.65$ & $1.77 \pm 0.77$ & $2.13 \pm 0.81$ \\
Enclosing Radius $(\boldsymbol{\mu m})$ & $134.6 \pm 60.7$ & $197.7 \pm 71.8$ & $251.9 \pm 95.3$ \\
\hline
\end{tabular}



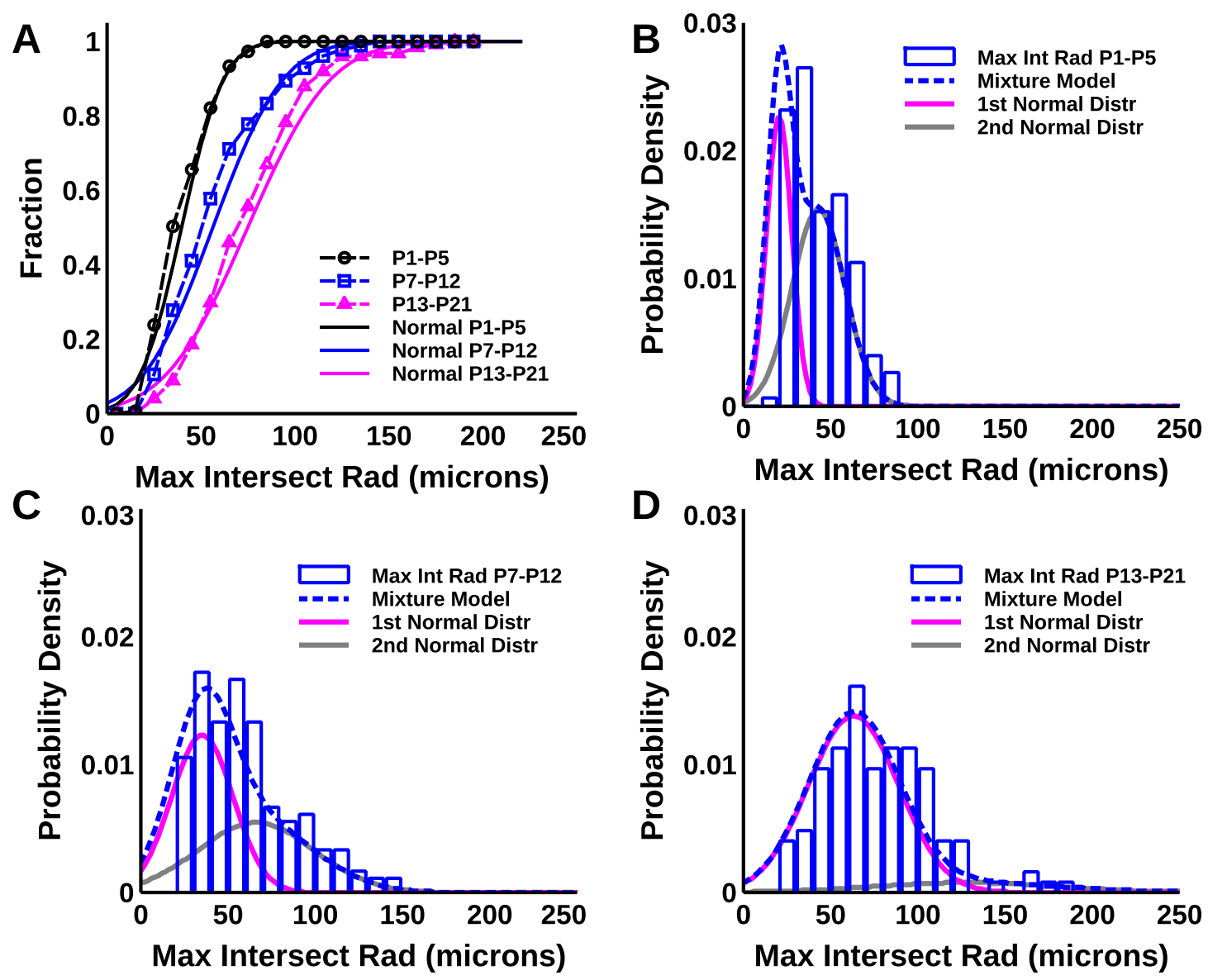

Figure 16. Pooled distributions for the maximum intersection radius showing the finite mixture distribution models. a) Cumulative and corresponding normal distributions for maximum intersection radius at P1-P5 (P1, P3, \& P5), P7-P12 (P7, P10, \& P12), and P13P21 (P13, P17, \& P21). b-d) Histograms for the maximum intersection radius and mixture distribution models showing the two normal distributions for b) P1-P5, c) P7-P12, and d) P13-P21. The parameters for the two normal distributions are given in Tables 5, 6. and 7. 

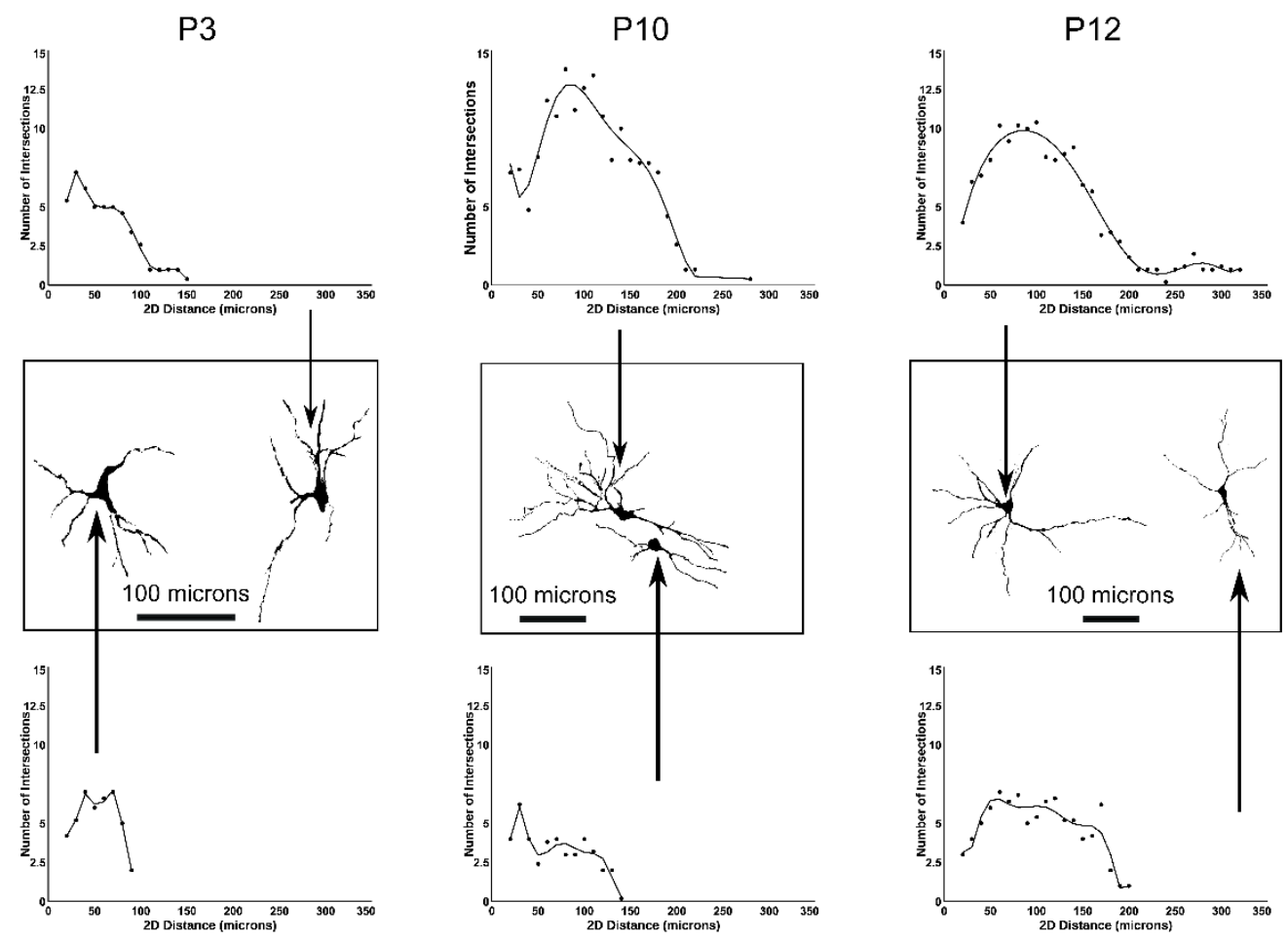

Figure 17. Threshold images of $\mathrm{P} 1, \mathrm{P} 10$, and $\mathrm{P} 12$ sections which have two isolated hypoglossal neurons with different morphologies, yet the centers of any two soma were with 50 to 200 microns in distance from each other. The micrograph of the XII MNs are shown with the Sholl profiles for the two neurons and arrows pointing to the respective neuron for each profile. 


\section{Discussion}

In this study we found that the morphology of developing hypoglossal motoneurons appears to be more complex than indicated by previous studies and age dependent. For each of the three time windows the morphology seemed best represented by a mixture of two normal distributions suggesting that there are at least two distinguishable phenotypes of XII MNs during the first three weeks post-birth. Our data also suggests that significant differences in morphometry of XII MNs can begin as early as $\mathrm{P} 7$, but most of the largest changes seem to occur at $\mathrm{P} 10-\mathrm{P} 13$ providing morphometric support for a "sensitive period." Statistical analyses of the morphological properties of 460 XII MNs across nine postnatal ages revealed three discrete developmental windows:

1) P1-P5; 2) P7-P12; and 3) P13-P21 and suggest that morphological changes proceeded significant functional changes (Liu et al., 2006, 2009; Wong-Riley et al., 2013; WongRiley and Liu, 2005). The finding of three discrete development windows supports our hypothesis in that there is a transition period (P7-P12) between the early (P1-P5) and the late (P13-P21) developmental periods. However, our results suggest that this transitional period may start sometime between P7 and P10. Although we did examine P7, we did not examine morphology at P8 or P9, so it is uncertain exactly when the transition window begins. These findings uniquely provide morphological evidence supporting developmental windows that may have functional significance for critical periods of respiratory development in infants and represent the most comprehensive assessment of XII MN developmental changes in morphology yet undertaken (Carrascal et al., 2005; Kanjhan et al., 2016a; Liu et al., 2006; Nunez-Abades and Cameron, 1995; Wong-Riley et al., 2013). 
Our findings extend research by other investigators who have studied the morphology of a small number of XII MNs after intracellular neurobiotin filling (Kanjhan et al., 2016a; Nunez-Abades et al., 1994; Nunez-Abades and Cameron, 1995). These previous studies only filled one or two neurons for each $300 \mu \mathrm{m}$ thick specimen, so sampling per animal was limited and the morphology of neighboring XII MNs was not measured. Because we sampled more neurons per animal, we have a more representative neuronal population for detailed morphological description at each postnatal day. Our statistical approach to XII MN morphology represents the most comprehensive quantitative assessment of developmental morphology in rat XII MNs yet undertaken. We report for the first time, age-dependent distributions of our morphometric variables, that allow us to determine whether the data are normally and unimodally distributed which are critical assumptions in statistical analyses. Our results suggest that not all neurons increase in size and complexity at the same time, but that some neurons change earlier with neighboring neurons changing later possibly indicating a more complex developmental model.

Biometric data support our findings that analyzed XII MNs came from healthy rat pups since the mean body weights are comparable to previous reports from healthy Sprague-Dawley rats (Dotta and Mortola, 1992; Sant'Anna and Mortola, 2002, 2003) at all development stages. Therefore, litter size was not a confounding factor. Comparing differences between animals at each postnatal age we did not see any indications for stratification of any parameters between the sexes. Adult motoneuron soma are large (>19 $\mu \mathrm{m})$, multipolar and the predominant type in the hypoglossal nucleus (XII), but there are also smaller interneurons $(10-18 \mu \mathrm{m})$ which are round-to-oval in shape (Boone 
and Aldes, 1984; Cooper, 1981; McClung and Goldberg, 1999). Another study reported interneurons in the XII as fusiform or ovoid in shape with a major diameter of $17.3 \pm 2.5$ $\mu \mathrm{m}$ with a few primary dendrites from the center and both poles (Takasu and Hashimoto, 1988). Interneurons have previously been reported as distributed toward the lateral onefourth and ventral margin of the XII (Takasu and Hashimoto, 1988). In neonatal mice the XII MNs are smaller for the first 8 postnatal days, yet since postnatal days 9-13 were group and averaged we do not know if these smaller XII MNs persist later than 8 days (Kanjhan et al., 2016). Therefore, a threshold size of $18 \mu \mathrm{m}$ is the main criteria we used to define XII MNs, while shape and number of primary branches severed as secondary criteria. We found XII MNs representing two different shapes (Type I and II) as previously described in adult cats (Fukunishi et al., 1999). It has been suggested that the Type II XII MN dendrites in the cat are confined to the nucleus while the Type I have dendrites that are extranuclear (external) suggesting that Type I neurons receive inputs in addition to those that terminate within the XII suggesting a functional difference (Fukunishi et al., 1999). Our data suggests that these Type I and Type II XII MNs are present in rats during the first three weeks post-birth. However, due the enclosing radii being only 100 to $150 \mu \mathrm{m}$, our results suggest these two neuronal types are present early, but the extranuclear extension of the Type I dendrites probably occur later (>P7).

\section{Postnatal Changes in Soma Morphology with Age}

The soma size of XII MNs increased progressively over the first three postnatal weeks, approaching adult size $(>19 \mu \mathrm{m})$ by $\mathrm{P} 21$, consistent with recently reported data in mice (Kanjhan et al., 2016a) with little change in shape as indicated by aspect ratio and 
form factor. In rats and mice, pups are weaned at P21 and begin sexual maturation (adolescence in humans) leading to adulthood (Dutta and Sengupta, 2016; Sengupta, 2013). Although previous studies have not tested their data for normality (normal distribution), we found that normality depends on the age and specific parameter investigated. Hartigan's dip test for a unimodal distribution (single mode; not bimodal or multimodal) revealed that parameters for soma size and shape at each age were unimodal, but the Shapiro-Wilk test for normality support that specific parameters at specific ages were not normally distributed (Ghasemi and Zahediasl, 2012; Hartigan and Hartigan, 1985; Le Boedec, 2016). Our findings provided criteria for pooling the somata (by size) into three groups that represented different stages in soma morphology based on nonparametric comparisons and visual inspection of cumulative distributions. These groups were pooled into (1) P1, P3, and P5; (2) P7, P10, and P12; and (3) P13, P17, and P21. Interestingly, neuron location did not explain the separation of the soma size into two distributions since there was no significant $(p<0.05)$ difference between the locations for either ECD or major diameter. The finite mixture distribution models suggest two subgroups of neurons with one sub-group representing a smaller percentage of the total number of neurons yet causing a right skew in the overall distribution. Because the proportions (percentage of the total data represented by each distribution) of the two distributions are not constant across the three age groups, the mixed distributions appeared to best characterize size shift—more neurons with larger soma—with increasing age. One possible explanation for this second distribution is that these neurons may be growing faster, earlier and the loss of this sub-group could relate to a delay or reduced 
rate of development as has been suggested for perinatal nicotine exposure (Powell et al., 2016).

\section{Postnatal Changes in Dendrite Morphology}

Dendrite morphology showed statistically significant age-dependent changes in both size and complexity. The size and complexity of the dendritic tree arbor relates functionally to the synaptic connectivity (Ferrante et al., 2013; Nunez-Abades et al., 1994; Kanjhan et al., 2016). The premotor neuron connections can be either excitatory or inhibitory while connections by interneurons in the nucleus are excitatory (Kanjhan et al., 2016). Little is known about the functional significance of the symmetry or asymmetry of dendritic arbors, but the asymmetry reported in this study has been previously reported for XII MNs in rats and mice (Nunez-Abades et al., 1994; Kanjhan et al., 2016). We found that both the enclosing radius and radius at the maximum number of intersections increased with age, indicating increasing dendritic tree arborization with increasing age. The maximum number of intersections rose during P1-P5 and during P7-P12 without affecting the ramification index (maximum number of intersections/number of primary branches). Still, at P7-P12, both parameters (maximum number of intersections and number of primary branches) were higher than observed at P1-P5. At these two developmental windows, the increase in the maximum number of intersections revealed by Sholl analysis is best explained by an increase in the number of primary branches. In contrast, at P13-P21, the increased maximum number of intersections and ramification index (compared to P1-P5) cannot be explained by increased primary branching. Instead, our results suggest an increased maximum intersection number at P13-P21 when compared to P1-P5. This was mainly due to an increase in the number of primary 
branches, while for P13-P21, the increase in maximum number of intersections was mainly due to increased branching distal to the primary dendrites. Therefore, our data show that size and complexity of the dendritic tree increased with age, but the change in complexity at P7-P12 was due to different processes (increased primary branching) than observed at P13-P21 (increased distal branching). The enclosing radius in the ventrallateral region of the XII was significantly lower (29\%) than the three regions in the P1P5 group.

Dendritic spines were observed on most XII MNs across all ages as previously reported for rats (Nunez-Abades et al., 1994) and mice (Kanjhan et al., 2016). Filopodia appeared more prominent at the earlier ages (P1-P7), decreasing with older neurons consistent with previous reports (Kanjhan et al., 2016b; Nunez-Abades et al., 1994). Dendritic spine morphology and density will be evaluated in a follow-up study. For all ages we also observed XII MNs with dendrites crossing the midline into the contralateral XII and neurons with dendrites extending outside the XII nucleus as has been previously reported (Kanjhan et al., 2016a; Koizumi et al., 2008; Nunez-Abades et al., 1994). We also observed that most of the dendrites of a neuron differed in their length and branching across the different primary branches as has been previously described by others (NunezAbades et al., 1994).

\section{Hypoglossal Motoneuron Morphology: Normality, Unimodality, and Distribution}

Since the "sensitive period" represents a transitional time, any change in the distribution of parameters between age groups - such as differences in the mean, the type 
of distribution, or a characteristic of the distribution (normal, unimodal, skewness, etc....) - may be reflected in functional changes. In this study we utilized tests for normality and unimodality to determine key characteristics relating to the morphology data we obtained from the XII MNs we analyzed. The Shapiro-Wilk test for normality (Ghasemi and Zahediasl, 2012; Le Boedec, 2016) showed that some of the parameters at specific ages or age ranges were not normally distributed. When a data set is nonnormally distributed, it is possible that it is also not unimodal. Despite statistical significance, changes in the characteristics of the distribution (normality and unimodality) may indicate differences between groups. Regardless of age or morphometric parameter, the morphology is unimodally distributed at all ages except for the maximum intersection radius for ages $\mathrm{P} 1-\mathrm{P} 12$. However, when the age groups were pooled, the maximum intersection radius was not unimodal for all three age ranges while for the enclosing radius the P7-P12 was not unimodal. The non-normal distribution of different parameters justified our use of the Kruskal-Wallis test and the Dunn test (García et al., 2010). The ratio of two normally distributed variables can result in a non-normal distribution (Marsaglia, 2006) which provides the simplest explanation for why the aspect ratio and ramification index are not normally distributed for most ages.

From testing three single distribution models (normal, log-normal, and gamma) as well as a finite mixture model (two normal distributions) we found that the finite mixture model was more consistent across the three age groups for most parameters. The only exception appeared to be the form factor for P1-P5 which tested for normality and a single normal distribution was the better fit, however for P7-P12 and P13-P21 the finite mixture model was the better fit. Some parameters such as the maximum number of 
intersections for P10 neurons fit a log-normal distribution as well (or better) than the mixed model of two normal distributions. However, other parameters such as the radius of the maximum number of intersections did not fit a log-normal distribution yet fit the mixed model. Overall, for the non-normally distributed data, the mixture model of two normal distributions appeared to be the best and most consistent fit for the data. This may not represent different types of neurons since the mean, standard deviations, and proportions are all changing, essentially continuously, with age. Topographical location could be the explanation, but there were no topological differences found for the maximum intersection radius. It may be that not all XII MNs are changing at the same rate with some somata and dendrites growing at earlier while other neurons are changing later. This was observed for most ages in that a given neuron had larger, more complex dendritic tree while another nearby neuron could have a smaller, less complex dendritic tree.

Another implication of these mixture distributions is that the development of XII MNs may not be a simple uniform change in morphology, but a more complex sequence of intermediate, punctuated changes. Although little is known or understood about what influences dendrite growth, previous work has suggested that axonal connectivity and other factors can affect dendrite length (Cowan et al., 1984; Easter et al., 1985; Fogarty et al., 2016, 2016; Guadiana et al., 2013; Purves and Lichtman, 1980). Other investigators have proposed mathematical and computational models for neuron and dendritic growth, but they have not examined nonuniform or staggered growth in neighboring neurons (Marks and Burke, 2007a, 2007b; Zubler and Douglas, 2009). Therefore, dendritic growth during early postnatal development may not be linear as previously suggested by 
others (Nunez-Abades et al., 1994; Nunez-Abades and Cameron, 1995) or uniform across neighboring XII MNs.

\section{Comparison with Previous Studies}

There are discrepancies in the existing literature regarding the age-related changes in soma and dendrite growth. Our study found progressive increases in soma size while dendrites increased in both size and complexity with increasing age, and we identified three distinct age groups for most morphometric parameters. Kanjhan and colleagues (2016) found changes in dendrite morphology and dendritic spine distribution in XII MNs from embryonic age E18 through postnatal age P28 in mice. Although there are morphological changes in somatic and dendritic morphology across age, most electrophysiological changes in motoneurons have been attributed to changes or differences in the type, subfamily, and distribution of ion channels (Carrascal et al., 2005). Somata size increases during embryonic and early postnatal life in mice (Kanjhan et al., 2016a), although it has been suggested that soma size reaches typical adult sizes by birth in cat and rat (Cameron et al., 1991; Nunez-Abades et al., 1994; Nunez-Abades and Cameron, 1995). However, Kanjhan and colleagues (2016a) measured a 70\% increase in soma volume from E17-P0 to P14-P28 while the studies in cat and rat only measured soma area by elliptical approximations. Our study agrees with Kanjhan and coworkers (2016a) in that we found that the equivalent circular diameter of the soma increased $40 \%$ from P1-P5 to P13-P21. One possible reason for the previous studies (Nunez-Abades and Cameron, 1995) not finding a difference in somata their small sample sizes (typically $\mathrm{N}=10$ neurons per age group) that limited the power of this study. In contrast, the study

on mice (Kanjhan et al., 2016) had an average of about 20 neurons per age group and our 
study had an average of approximately 150 neurons for each of the three age groups. For each of the nine postnatal ages we had 30 to 60 neurons for each age providing sufficient statistical power.

For mice the total dendrite length increased by approximately $26 \%$ from P1-P4 to P9-P13 (Kanjhan et al., 2016a) and in rats the maximum dendrite length increased by about 36\% from P1-P2 to P19-P30 (Nunez-Abades et al., 1994). Our study found that the enclosing radius increased about $89 \%$ from P1-P5 to P13-P21. The mean dendrite length in the other two studies was around $300 \mu \mathrm{m}$, but the mean enclosing radius in our study was approximately $135 \mu \mathrm{m}$ for P1-P5. The other two studies (Kanjhan et al., 2016a; Nunez-Abades et al., 1994) used ionophoretic injections of neurobiotin while our study employed Golgi-Cox staining resulting in the measurement of more neurons per animal as well as allowing multiple neighboring neurons to be measured in the same sections.

We also found that non-parametric comparisons supported no significant differences allowing pooling of the data into three age ranges (P1-P5, P7-P12, and P13P21). One previous study in mice (Kanjhan et al., 2016a) grouped data into five groups (E17-P0, P1-P4, P5-P8, P9-P13, and P14-P28) while another study in rats (NunezAbades et al., 1994; Nunez-Abades and Cameron, 1995) used four groups (P1-P2, P5P6, P13-P15, and P19-P30). Our data suggests that grouping ages between P1 and P6 and $\mathrm{P} 13-15$ is appropriate but grouping P5-P8 or P9-P13 may result in missing important morphological differences that we have noted in our analysis. Previous studies (Kanjhan et al., 2016a; Nunez-Abades et al., 1994; Nunez-Abades and Cameron, 1995) 
have not been focused on the functional impact of changes in XII MN morphology from the perspective of respiratory physiology.

\section{Impact of XII Morphology on Respiratory Function}

In addition to respiration, the tongue and upper airways play critical roles in speech, an important aspect of motor control that motivates our study of XII MN development (Kanjhan et al., 2016a; Nunez-Abades et al., 1994; Nunez-Abades and Cameron, 1995). In spite of the breathing phenotype changes previously reported for days 10 to 13 (Liu et al., 2006, 2009; Wong-Riley et al., 2013; Wong-Riley and Liu, 2005)), there has been no comprehensive longitudinal investigation into the morphology of developing XII MNs prior to this study. The developmental pattern in respiratory regulation and control has been investigated (Liu et al., 2006, 2009) demonstrating the afore-mentioned sensitive period between P10 and P13 days post-birth where the hypoxic ventilatory response is altered. Wong-Riley and Liu (2005) have also found a shift in excitatory and inhibitory neurotransmitter expression during the P10-P13 sensitive period at P12.

Neurotransmitters such as glutamate, GABA, glycine and catecholamines have been shown to change during development with pronounced shifts in expression during the sensitive period (Brust et al., 2014; Wong-Riley and Liu, 2005). Therefore, it is suggested that the sensitive period represents a transition period between the early postnatal ages $(>P 0)$ and the later ages $(>\mathrm{P} 13)$ in which the animal is vulnerable to any event that could interfere with development. A recent study of postnatal exposure to lipopolysaccharide (LPS) in rats demonstrated that there was a heightened vulnerability to endotoxin exposure at P10, characterized by a depressed ventilatory response to acute 
hypoxia when compared with P5 and P20 (Rourke et al., 2016). This study also demonstrated increased inflammation and mortality in LPS-treated rats at P10 when compared with P5 and P20. This is one of the first studies to demonstrate that the sensitive period from P10 through P13 represents not only a window of time in which abrupt changes occur, but a vulnerable window in which heightened susceptibility to infection can occur. Another recent study of perinatal nicotine exposure showed morphological changes in XII MNs for P0-P4 but did not extend to P10-P13 (Powell et al., 2016). Thus, there are very few, if any studies that have examined respiratory or related phenomena for perinatal nicotine exposure or maternal inflammation during P10P13. In our study dendrite morphologies showed increases in morphometric parameters from $\mathrm{P} 1-\mathrm{P} 5$ to $\mathrm{P} 13-\mathrm{P} 21$ with the $\mathrm{P} 7-\mathrm{P} 12$ representing a transition period between the early and later developmental stages. Now that we have established a detailed pattern of morphological development differences in XII MNs due to perinatal nicotine exposure or maternal inflammation could be elucidated.

\section{Limitations and Future Directions}

In this study we have shown that soma and dendrite morphology changes with postnatal age; however, changes in 3D morphometric parameters such as dendrite length, branching, and dendritic spines, were not examined. Therefore, based on the complexity of changes in the parameters with age seen in this study it may be of benefit to examine the 3D morphometric parameters and changes in synaptic spines to correlate morphology and function in the XII. Using a larger number of animals to yield sufficient numbers of neurons $(\mathrm{N}>60)$ at specific individual ages to test the models would provide more extensive data regarding the distributions and changes between individual ages for 
specific morphometric parameters. Three-dimensional reconstruction with modeling of the biophysical properties based on the 3D geometries in concert with electrophysiological measurements would provide a foundation for understanding the relationships between structure and function at the neuron level. An additional limitation is we are unable to address the targets of the XII MNs, because when cutting brain slices the cranial rootlets were cut proximal to the brainstem - making it impossible for us to address the number of motor units targeted by each XII MN. Future experiments therefore need to be performed in which the innervation of the tongue is retained and the connection from brainstem to tongue would allow us to assess which motoneurons innervate a given motor unit in the tongue, however, this is beyond the scope of the present study. Additionally, understanding the pattern of synaptic inputs to XII MNs would further our understanding of the role of developmental changes in respiratory control circuit. Despite these limitations, the detailed overview of developmental changes in XII MNs that we present in this study provides a foundation for future developmental morphology work in the XII nucleus.

\section{Summary and Conclusions}

We have shown that the soma size of XII MNs increases with postnatal age, but there is little, if any change in shape of the somata. In contrast, the dendritic arbor of XII MNs increases in size and complexity as postnatal age increases with increased branching, a larger maximum radius, and an increase in the radius at which the maximum number of intersections occurs. Three "stages" of development were identified for the morphology of XII MNs in this study with P1-P5, P7-P12, and P13-P21 being morphologically distinct. The functional sensitive period previously described is reflected 
morphologically in the maturation of XII MNs especially for the maximum number of intersections and enclosing radius. Finally, our observations suggest that the development of XII MNs is more complex than previously described and further investigation correlating structure with function is needed. These findings and the detailed description of XII MN development in the first stages post-birth provides a better understanding of crucial windows during the development of respiratory control circuitry.

\section{Acknowledgements}

Imaging was performed in the LLUSM Advanced Imaging and Microscopy Core with support from National Science Foundation grant MRI-DBI 0923559 and Loma Linda University School of Medicine. The authors thank Johnny Figueroa, $\mathrm{PhD}$ for assistance and use of the Keyence microscope. 


\section{Literature Cited}

Boone, T.B., Aldes, L.D., 1984. The ultrastructure of two distinct neuron populations in the hypoglossal nucleus of the rat. Exp Brain Res 54, 321-6.

Brust, R.D., Corcoran, A.E., Richerson, G.B., Nattie, E., Dymecki, S.M., 2014. Functional and developmental identification of a molecular subtype of brain serotonergic neuron specialized to regulate breathing dynamics. Cell Rep. 9, 2152-2165.

Cameron, W.E., He, F., Kalipatnapu, P., Jodkowski, J.S., Guthrie, R.D., 1991. Morphometric analysis of phrenic motoneurons in the cat during postnatal development. J Comp Neurol 314, 763-76.

Carrascal, L., Nieto-Gonzalez, J.L., Cameron, W.E., Torres, B., Nunez-Abades, P.A., 2005. Changes during the postnatal development in physiological and anatomical characteristics of rat motoneurons studied in vitro. Brain Res Brain Res Rev 49, 377-87.

Cooper, M.H., 1981. Neurons of the hypoglossal nucleus of the rat. Otolaryngol Head Neck Surg 89, 10-5.

Cowan, W.M., Fawcett, J.W., O’Leary, D.D., Stanfield, B.B., 1984. Regressive events in neurogenesis. Science 225, 1258-65.

Das, G., Reuhl, K., Zhou, R., 2013. The Golgi-Cox method. Methods Mol Biol 1018, $313-21$.

Delignette-Muller, M.L., Dutang, C., 2015. fitdistrplus: An R Package for Fitting Distributions. J. Stat. Softw. Vol 1 Issue 420151.

Dotta, A., Mortola, J.P., 1992. Postnatal development of the denervated lung in normoxia, hypoxia, or hyperoxia. J Appl Physiol 1985 73, 1461-6.

Dutta, S., Sengupta, P., 2016. Men and mice: Relating their ages. Life Sci 152, 244-8.

Easter, S.S., Jr., Purves, D., Rakic, P., Spitzer, N.C., 1985. The changing view of neural specificity. Science 230, 507-11.

Erickson, J.T., Shafer, G., Rossetti, M.D., Wilson, C.G., Deneris, E.S., 2007. Arrest of 5HT neuron differentiation delays respiratory maturation and impairs neonatal homeostatic responses to environmental challenges. Respir. Physiol. Neurobiol. 159, 85-101.

Everitt, B.S., 1996. An introduction to finite mixture distributions. Stat Methods Med Res 5, 107-27.

Feldman, J.L., Del Negro, C.A., 2006. Looking for inspiration: new perspectives on 
respiratory rhythm. Nat. Rev. Neurosci. 7, 232-242.

Feldman, J.L., Del Negro, C.A., Gray, P.A., 2013. Understanding the rhythm of breathing: so near, yet so far. Annu. Rev. Physiol. 75, 423-452. Ferrante, M., Migliore, M., Ascoli, G.A., 2013. Functional impact of dendritic branch-point morphology. J Neurosci 33, 2156-65.

Ferrante, M., Migliore, M., \& Ascoli, G. A., 2013. Functional impact of dendritic branchpoint morphology. J Neurosci, 33(5), 2156-2165. doi:10.1523/JNEUROSCI.3495-12.2013

Ferreira, T.A., Blackman, A.V., Oyrer, J., Jayabal, S., Chung, A.J., Watt, A.J., Sjostrom, P.J., van Meyel, D.J., 2014. Neuronal morphometry directly from bitmap images. Nat Meth 11, 982-984.

Fietkiewicz, C., Loparo, K.A., Wilson, C.G., 2011. Drive latencies in hypoglossal motoneurons indicate developmental change in the brainstem respiratory network. J. Neural Eng. 8.

Fogarty, M.J., Kanjhan, R., Bellingham, M.C., Noakes, P.G., 2016. Glycinergic Neurotransmission: A Potent Regulator of Embryonic Motor Neuron Dendritic Morphology and Synaptic Plasticity. J Neurosci 36, 80-7.

Forbes, C., Evans, M., Hastings, N., Peacock, B., 2011. Stochastic Modeling, in: Statistical Distributions. Hoboken, New Jersey, pp. 32-43.

Fukunishi, Y., Nagase, Y., Yoshida, A., Moritani, M., Honma, S., Hirose, Y., Shigenaga, Y., 1999. Quantitative analysis of the dendritic architectures of cat hypoglossal motoneurons stained intracellularly with horseradish peroxidase. J Comp Neurol 405, 345-58.

Fulton, B.P., Walton, K., 1986. Electrophysiological properties of neonatal rat motoneurones studied in vitro. J Physiol 370, 651-78.

García, S., Fernández, A., Luengo, J., Herrera, F., 2010. Advanced nonparametric tests for multiple comparisons in the design of experiments in computational intelligence and data mining: Experimental analysis of power. Inf. Sci. 180, 20442064.

Ghasemi, A., Zahediasl, S., 2012. Normality tests for statistical analysis: a guide for nonstatisticians. Int J Endocrinol Metab 10, 486-9.

Gibb, R., Kolb, B., 1998. A method for vibratome sectioning of Golgi-Cox stained whole rat brain. J Neurosci Methods 79, 1-4.

Guadiana, S.M., Semple-Rowland, S., Daroszewski, D., Madorsky, I., Breunig, J.J., Mykytyn, K., Sarkisian, M.R., 2013. Arborization of dendrites by developing neocortical neurons is dependent on primary cilia and type 3 adenylyl cyclase. $\mathrm{J}$ 
Neurosci 33, 2626-38.

Harper, R.M., Kinney, H.C., 2010. Potential Mechanisms of Failure in the Sudden Infant Death Syndrome. Curr. Pediatr. Rev. 6, 39-47.

Hartigan, J.A., Hartigan, P.M., 1985. The Dip Test of Unimodality. Ann. Stat. 13, 70-84.

Kanjhan, R., Fogarty, M.J., Noakes, P.G., Bellingham, M.C., 2016a. Developmental changes in the morphology of mouse hypoglossal motor neurons. Brain Struct. Funct. 221, 3755-3786.

Kanjhan, R., Noakes, P.G., Bellingham, M.C., 2016b. Emerging Roles of Filopodia and Dendritic Spines in Motoneuron Plasticity during Development and Disease. Neural Plast. Volume 2016, Article ID 3423267, 31 pages.

Khazipov, R., Zaynutdinova, D., Ogievetsky, E., Valeeva, G., Mitrukhina, O., Manent, J.B., Represa, A., 2015. Atlas of the Postnatal Rat Brain in Stereotaxic Coordinates. Front. Neuroanat. 9.

Kinney, H.C., 2009. Brainstem mechanisms underlying the sudden infant death syndrome: evidence from human pathologic studies. Dev Psychobiol 51, 223-33.

Koizumi, H., Wilson, C.G., Wong, S., Yamanishi, T., Koshiya, N., Smith, J.C., 2008. Functional Imaging, Spatial Reconstruction, and Biophysical Analysis of a Respiratory Motor Circuit Isolated In Vitro. J. Neurosci. 28, 2353-2365.

Komendantov, A.O., Ascoli, G.A., 2009. Dendritic excitability and neuronal morphology as determinants of synaptic efficacy. J Neurophysiol 101, 1847-66.

Le Boedec, K., 2016. Sensitivity and specificity of normality tests and consequences on reference interval accuracy at small sample size: a computer-simulation study. Vet Clin Pathol 45, 648-656.

Lindsey, B.G., Rybak, I.A., Smith, J.C., 2012. Computational models and emergent properties of respiratory neural networks. Compr Physiol 2, 1619-70.

Liu, Q., Fehring, C., Lowry, T.F., Wong-Riley, M.T., 2009. Postnatal development of metabolic rate during normoxia and acute hypoxia in rats: implication for a sensitive period. J Appl Physiol 106, 1212-22.

Liu, Q., Lowry, T.F., Wong-Riley, M.T.T., 2006. Postnatal changes in ventilation during normoxia and acute hypoxia in the rat: implication for a sensitive period. J Physiol 577, 957-70.

Liu, Q., Wong-Riley, M.T., 2003. Postnatal changes in cytochrome oxidase expressions in brain stem nuclei of rats: implications for sensitive periods. J Appl Physiol 95, 2285-91. 
Liu, Q., Wong-Riley, M.T.T., 2006. Developmental changes in the expression of GABAA receptor subunits alpha1, alpha2, and alpha3 in brain stem nuclei of rats. Brain Res 1098, 129-38.

Liu, Q., Wong-Riley, M.T.T., 2002. Postnatal expression of neurotransmitters, receptors, and cytochrome oxidase in the rat pre-Botzinger complex. J. Appl. Physiol. 92, 923-934.

Liu, Y.-Y., Wong-Riley, M.T.T., 2001. Developmental study of cytochrome oxidase activity in the brain stem respiratory nuclei of postnatal rats. J. Appl. Physiol. 90, 685-694.

Marks, W.B., Burke, R.E., 2007a. Simulation of motoneuron morphology in three dimensions. I. Building individual dendritic trees. J Comp Neurol 503, 685-700.

Marks, W.B., Burke, R.E., 2007b. Simulation of motoneuron morphology in three dimensions. II. Building complete neurons. J Comp Neurol 503, 701-16.

Marsaglia, G., 2006. Ratios of Normal Variables. J. Stat. Softw. 16, 10.

Martin, R.J., Wilson, C.G., 2009. What to do about apnea of prematurity? J Appl Physiol 107, 1015-6.

McClung, J.R., Goldberg, S.J., 1999. Organization of motoneurons in the dorsal hypoglossal nucleus that innervate the retrusor muscles of the tongue in the rat. Anat Rec 254, 222-30.

Mortola, J.P., 1984. Breathing pattern in newborns. J Appl Physiol Respir Env. Exerc Physiol 56, 1533-40.

Nunez-Abades, P.A., Cameron, W.E., 1995. Morphology of developing rat genioglossal motoneurons studied in vitro: relative changes in diameter and surface area of somata and dendrites. J Comp Neurol 353, 129-42.

Nunez-Abades, P.A., He, F., Barrionuevo, G., Cameron, W.E., 1994. Morphology of developing rat genioglossal motoneurons studied in vitro: changes in length, branching pattern, and spatial distribution of dendrites. J Comp Neurol 339, 40120.

Nunez-Abades, P.A., Spielmann, J.M., Barrionuevo, G., Cameron, W.E., 1993. In vitro electrophysiology of developing genioglossal motoneurons in the rat. $\mathrm{J}$ Neurophysiol 70, 1401-11.

Powell, G.L., Gaddy, J., Xu, F., Fregosi, R.F., Levine, R.B., 2016. Developmental Nicotine Exposure disrupts dendritic arborization patterns of hypoglossal motoneurons in the neonatal rat. Dev Neurobiol.

Preibisch, S., Saalfeld, S., Tomancak, P., 2009. Globally optimal stitching of tiled 3D 
microscopic image acquisitions. Bioinformatics 25, 1463-1465.

Purves, D., Lichtman, J., 1980. Elimination of synapses in the developing nervous system. Science 210, 153-157.

Purvis, L.K., Butera, R.J., 2005. Ionic Current Model of a Hypoglossal Motoneuron. J. Neurophysiol. 93, 723-733.

Rourke, K.S., Mayer, C.A., MacFarlane, P.M., 2016. A critical postnatal period of heightened vulnerability to lipopolysaccharide. Respir Physiol Neurobiol 232, 2634.

Sant’Anna, G.M., Mortola, J.P., 2003. Thermal and respiratory control in young rats exposed to cold during postnatal development. Comp Biochem Physiol Mol Integr Physiol 134, 449-59.

Sant’Anna, G.M., Mortola, J.P., 2002. Thermal and respiratory control in young rats with altered caloric intake during postnatal development. Respir Physiol Neurobiol 133, 215-27.

Sengupta, P., 2013. The Laboratory Rat: Relating Its Age With Human’s. Int J Prev Med 4, 624-30.

Sholl, D.A., 1950. A discussion on the measurement of growth and form; the theory of differential growth analysis. Proc R Soc Lond B Biol Sci 137, 470-4.

Smith, J.C., Butera, R.J., Koshiya, N., Negro, C.D., Wilson, C.G., Johnson, S.M., 2000. Respiratory rhythm generation in neonatal and adult mammals: the hybrid pacemaker-network model. Respir. Physiol. 122, 131-147.

Smith, J.C., Ellenberger, H.H., Ballanyi, K., Richter, D.W., Feldman, J.L., 1991. PreBötzinger complex: a brainstem region that may generate respiratory rhythm in mammals. Science 254, 726-729.

Standard Practice for Characterization of Particles (No. F 1877-98), 2003. ASTM.

Takasu, N., Hashimoto, P.H., 1988. Morphological identification of an interneuron in the hypoglossal nucleus of the rat: a combined Golgi-electron microscopic study. J Comp Neurol 271, 461-71.

Thoby-Brisson, M., Trinh, J.-B., Champgagnat, J., Fortin, G., 2005. Emergence of the pre-Bötzinger respiratory rhythm generator in the mouse embryo. J. Neurosci. 25, 4307-4318.

Wong-Riley, M.T., Liu, Q., 2005. Neurochemical development of brain stem nuclei involved in the control of respiration. Respir Physiol Neurobiol 149, 83-98.

Wong-Riley, M.T., Liu, Q., Gao, X.P., 2013. Peripheral-central chemoreceptor interaction 
and the significance of a critical period in the development of respiratory control. Respir Physiol Neurobiol 185, 156-69.

Zubler, F., Douglas, R., 2009. A Framework for Modeling the Growth and Development of Neurons and Networks. Front. Comput. Neurosci. 3, 25. 


\title{
CHAPTER THREE
}

\section{MODELING HYPOGLOSSAL MOTONEURONS IN THE DEVELOPING RAT}

\author{
Paul Allen Williams ${ }^{1,2}$, Clarissa Dalton ${ }^{3}$, Christopher G. Wilson ${ }^{1,2}$ \\ ${ }^{1}$ Division of Physiology, Basic Science Department \\ ${ }^{2}$ Lawrence D. Longo, MD Center for Perinatal Biology, \\ Loma Linda University School of Medicine \\ 11234 Anderson St, Loma Linda, California 92350 \\ ${ }^{3}$ California Polytechnic State University - San Luis Obispo \\ San Luis Obispo, California
}

Respiratory Physiology and Neurobiology, 2018, In review

(Modified version) 


\begin{abstract}
At birth, the autonomic brainstem, which generates and controls respiratory rhythm for normal breathing is not fully developed. Normal control of breathing develops in the first few weeks after birth. However, little is known about changes in the morphology and electrophysiological behavior of neurons in the respiratory regions of the brainstem during this early developmental period. We hypothesize that developmental changes in the morphology of motoneurons in the hypoglossal motor nucleus (XII) can lead to changes in biophysical properties that alter motor output. To test this hypothesis, we used Golgi-Cox staining to stain neurons in the brainstem and we quantified the morphology of the somata and dendritic tree of developing hypoglossal XII motoneurons (XII MNs) using Sholl analysis. We then used the computer simulation environment, NEURON, to model the biophysical properties from the digitally reconstructed neurons. At nine postnatal ages $(\mathrm{P} 1,3,5,7,10,12,13,17$, and 21$)$, we removed the brain from Sprague-Dawley rat pups, processed the tissue, sectioned, stained, imaged, and performed 3D reconstructions to quantify the morphometrics of XII MNs. We then used these $3 \mathrm{D}$ reconstructed geometries to model the biophysical properties of the neurons. The soma of XII MNs increases in size by approximately 50\% from P1 to P21. The dendritic trees extend further, and branching becomes more complex with most of the statistically significant changes starting at P10 or P12 when compared to P1, P3, and P5. Morphology correlated with the passive electrophysiological properties of XII MNs. Although a direct relationship was not found between morphology and the active properties, there was evidence of an indirect relationship in that the passive properties correlated with the active properties.
\end{abstract}




\section{Introduction}

Respiration is critical during development and throughout life and occurs rhythmically through an elaborate system of control. At birth, autonomic regions in the brainstem, which generates and controls respiratory rhythm are not fully developed (Feldman et al., 2013; Feldman and Del Negro, 2006; Feldman and Smith, 1995). Normal control of breathing develops in the first few weeks after birth. The motoneurons (MNs) of the hypoglossal nucleus (XII) and other brainstem nuclei provide the drive to the tongue and upper airways and are used in in vitro models to provide an index of fictive inspiratory effort (Feldman et al., 2013; Feldman and Del Negro, 2006). The critical role that the tongue and upper airway control plays in speech is also an important aspect that motivates the study of XII MN development. We have previously shown that there are age-dependent changes in XII MN output drive, but the contribution of developmental changes in neural morphology remains essentially unknown (Fietkiewicz et al., 2011). Because the XII MNs are easy to identify and play an active role in inspiration during development, they are a convenient and accessible population of neurons for electrophysiological study. Also, since $80 \%$ or more of the neurons in the XII are motoneurons that are readily distinguishable from the interneurons, the XII MNs are convenient for morphological studies (Cooper, 1981; McClung and Goldberg, 1999).

Work by others have shown that development of the respiratory network plays a key role in the changing of breathing pattern (Liu et al., 2006; Mortola, 1984).

Understanding the remodeling of the breathing neural network during early life is key to elucidating the synergy between changes in morphology and breathing rhythm in the perinatal period. It has been shown that neural network behavior is influenced by the morphology of neurons, and that connectivity is altered due to changes in 
neurotransmitter release as well as receptor up- and down-regulation (Ferrante et al., 2013; Komendantov and Ascoli, 2009). Hypoglossal motoneurons (XII MNs) which drive the tongue and upper airway musculature are used in in vitro models to provide an index of fictive inspiratory function (Koizumi et al., 2008; Purvis and Butera, 2005; Smith et al., 1991, 2000). Additionally, our laboratory has previously shown agedependent changes in the distribution of drive latencies in hypoglossal motoneuron output (Fietkiewicz et al., 2011). There is a body of work on the morphology and electrophysiology of motoneurons with some work on hypoglossal motoneurons and a few studies on morphological changes during postnatal development (Carrascal et al., 2005; Kanjhan et al., 2016; Lindsey et al., 2012; Nunez-Abades and Cameron, 1995). Morphological differences in XII MNs have been shown between neonatal and adults as well as corresponding electrophysiological changes (Berger et al., 1996; Carrascal et al., 2005). It has also been demonstrated that the morphology and electrophysiology of motoneurons differ for various regions in the central nervous system (Carrascal et al., 2005). Although most electrophysiological changes in MNs have been attributed to changes or differences in the type, subtype, and distributions of ion channels (Carrascal et al., 2005), other studies have shown a relationship between morphology and membrane properties that can influence electrophysiology (Nunez-Abades and Cameron, 1997). A recent study of XII MNs in mice found changes in dendrite morphology and dendritic spine distribution from embryonic age E18 through postnatal age P28 (Kanjhan et al., 2016). In addition, a few studies have utilized single compartment models (soma) to model various aspects of XII MN electrophysiology (Fietkiewicz et al., 2011; Purvis and Butera, 2005). However, most studies (morphological or electrophysiological) have 
examined only a single or small window of ages within the neonatal period, or group ages in a somewhat arbitrary manner (Kanjhan et al., 2016; Nunez-Abades and Cameron, 1995).

Therefore, there is a need to understand both electrophysiological behavior and morphology to assess the relationship between morphology and biophysical function in XII MNs. In this study we modeled passive and active electrophysiological properties based on actual morphological geometries of XII MNs. Our objective was to simulate actual electrophysiology experiments on morphologically accurate XII MNs and compare our simulation results with experimental results reported in the literature. Our hypothesis was that morphological characteristics can influence simulated passive and active electrophysiological behavior. We chose three ages ranging from the first, second, and third weeks of development. We utilized established single-compartment models and iterated the parameter values until experimental and computational results were comparable. Then we examined correlations between the electrophysiology and neuron morphology.

\title{
Material and Methods
}

\author{
Animals \\ Pregnant Sprague-Dawley dams (Charles River Laboratories International, Inc., \\ Wilmington, MA) obtained approximately 18 days after the start of pregnancy (E18) \\ were allowed to deliver naturally in the Loma Linda University Animal Care Facility. Rat \\ pups at nine postnatal ages (P1, P3, P5, P7, P10, P12, P13, P17, and P21 days) were \\ initially used in the study. Typical litter size was approximately 9-14 pups and no \\ distinction were made between genders. When two litters were used the two litters were
}


balanced to maintain consistent litter size (number of pups per litter) if needed. All procedures and protocols used in this study were approved by the Institutional Animal Care and Use Committee at Loma Linda University and followed the guidelines by the National Institutes of Health Guide for the Care and Use of Laboratory Animals (http://www.ncbi.nlm.nih.gov/books /NBK54050).

\section{Golgi-Cox Staining}

On the appropriate developmental day, animals were weighed, euthanized, and the brain extracted. For postnatal ages P1 through P7 the pups were anesthetized with isoflurane (Western Medical Supply, Arcadia, CA) until unresponsive to toe pinch, then their brains were rapidly extracted and placed in chilled $0.9 \%$ saline. For postnatal ages P10 through P21, animals were placed in an induction chamber with oxygen (100\%) flow at $0.65 \mathrm{~L} / \mathrm{min}$ and isoflurane administered at a concentration of 3 to $4 \%$ based on the postnatal age. Once the animal was unresponsive to toe pinch it was removed from the induction chamber, placed on a platform with the oxygen and isoflurane continuously administered via a nose cone, and perfused with $0.9 \%$ saline for approximately two minutes after which the brain was removed. For ages P1 through P7, the whole brain was maintained intact while for P10 through P21 the brain was sectioned coronally into two tissue blocks to maintain a more consistent tissue volume.

The brains were immediately transferred from $0.9 \%$ saline to impregnation solution from a commercial kit (superGolgi Kit, Bioenno Tech, LLC, Santa Ana, California). Sections impregnated at different time durations showed that the best results were obtained with 7 days of impregnation at room temperature changing the solution 
after the initial two days. Therefore, impregnation solution was changed after two days then the specimens were left in the solution for five more days according to the supplier's instructions and previous reported methods with all steps performed at room temperature (Das et al., 2013). After a total of 7 days in impregnation solutions the brains were placed in two changes of post-impregnation buffer (changed each day for two days). We performed coronal sectioning of tissue at $150 \mu \mathrm{m}$ with a vibratome (Vibratome Series 1000 Classic-Tissue Sectioning System, Leica, St. Louis, MO) mounting the sections onto gelatin-coated slides and then allowed to dry slowly in a humidified chamber overnight (Das et al., 2013; Gibb and Kolb, 1998). The slides were dried at room temperature and humidity for approximately one hour and then rehydrated in phosphate buffered saline, stained, placed in post-staining buffer, and then washed, dehydrated, and cleared in xylene as per the supplier's instructions and the literature (Das et al., 2013). After staining, Permount (Fisher Scientific, SP-15, Waltham, MA) was applied to the slides which were then cover-slipped and allowed to fully dry.

\section{Imaging}

The XII nucleus from each animal was identified by comparing brainstem sections imaged using a $2 \mathrm{x}$ objective with atlases (Khazipov et al., 2015) corresponding to the appropriate postnatal period. We used a 40x objective to acquire image stacks for each XII MN with one image every $2 \mu \mathrm{m}$ in the z-direction. For the P1-P7 animals a single image stack was obtained using a microscope (Ziess Axio Imager, Carl Zeiss Microscopy, LLC, Peabody, MA USA) with a motorized stage (Applied Scientific Instrumentation, Eugene, OR) and imaging software (Stereologer, Stereology Resource 
Center, Tampa FL). For the P10-P21 animals, we acquired either a single image stack or several overlapping image stacks (multiple stacks). Multiple stacks were required for many of the P10-P21 animals in order to image the entire dendritic morphology of a single neuron at 40x, so we used another microscope (Keyence, BZ-9000, Itasca, IL) to image these neurons.

\section{Mapping of Neuron Locations}

The position of each XII MN was measured with ImageJ to acquire the length and angle of a line from the apex of the fourth ventricle (rostral) or center of the central canal (caudal). These lengths and angles were converted to $\mathrm{x}$ (horizontal) and $\mathrm{y}$ (vertical) distances with $\mathrm{x}$ representing the medial-lateral and $\mathrm{y}$ representing the dorsal-ventral direction. These points were plotted relative to the anatomical reference point along with the boundary of the XII nucleus determined in a similar manner, as previously described (Kanjhan et al., 2016). For the rostral-caudal extent of XII, sections were matched with a representative rat atlas for each postnatal age and the lambda coordinate along with the $y$ axis (dorsal-ventral direction) was used to plot location in the sagittal plane similar to that previously described (Kanjhan et al., 2016; Khazipov et al., 2015). Due to size differences between the postnatal ages, lambda was normalized by the lambda value at the obex. Neurons located in the XII nucleus for both the coronal and sagittal maps were included while any neurons outside the XII were excluded from analysis. 


\section{Selection of Motoneurons}

The majority of neurons in the XII nucleus are MNs, yet there are a few interneurons (Boone and Aldes, 1984; Cooper, 1981; Takasu and Hashimoto, 1988). Due to the presence of these interneurons we needed criteria to determine if the measured neuron was a motoneuron. In the adult rat motoneurons are larger in diameter $(19-50 \mu \mathrm{m})$ than the interneurons (10-18 $\mu \mathrm{m})$ (Boone and Aldes, 1984; Cooper, 1981). For mouse XII MNs, it has been shown that the P1-P4 had a major diameter which was $25 \%$ lower than for the P14-P28 (Kanjhan et al., 2016). Therefore, our main criteria for determining a MN was that from $\mathrm{P} 1$ to $\mathrm{P} 7$ the size needed to be at least $18 \mu \mathrm{m}$ with the size being at least $20 \mu \mathrm{m}$ at P10 to P21. Adult interneurons have been described as fusiform or oval in shape with few primary dendrites from both poles and from the center, while motoneurons were multipolar in shape (Boone and Aldes, 1984; Takasu and Hashimoto, 1988). However, this has not been as well established for neonatal XII MNs (Kanjhan et

al., 2016; Nunez-Abades et al., 1994; Nunez-Abades and Cameron, 1995). Therefore, our main criteria for determining a $\mathrm{MN}$ was that from $\mathrm{P} 1$ to $\mathrm{P} 7$ the size needed to be at least $18 \mu \mathrm{m}$ with the size being at least $20 \mu \mathrm{m}$ at P10 to P21. Neuronal shape (oval, fusiform, multipolar) was then used as a secondary criterion (Boone and Aldes, 1984; Cooper, 1981; Fukunishi et al., 1999; Nunez-Abades et al., 1994; Nunez-Abades and Cameron, 1995; Takasu and Hashimoto, 1988).

\section{D Reconstructions}

For the P1 through P7 animals, single image stacks were obtained, however for the P10 through P21 animals, multiple stacks were stitched together using the ImageJ 
stitching plug-in (Preibisch et al., 2009). We obtained at least 5-7 pups per age from seven litters. Litter size ranged from 9 to 14 (average of about 12 pups per litter). A total number of 30 or more MNs were obtained for each postnatal age. From the image stacks 3D reconstructions were obtained For P3, P10, and P17 XII MNs using a software package (Neuromantic, Reading, UK) with volumes and surface areas measured (Myatt et al., 2012). For P3, P10, and P17 XII MNs with volumes equaled to or approximating the minimum, maximum, and average volume values for the soma or dendrites were selected. This provided XII MNs for each of the three ages with ten from the P3 XII MNs and six each from the P10 and P17 XII MNs resulting in a total of 22 neurons for simulation. Minimum, maximum, and average volumes were used to allow for the largest range of morphologies to accommodate the regression analysis. Although this decreased statistical power for comparison tests, a larger variance was more consistent for our objective and hypothesis which were focused on the regression analysis.

\section{Simulations}

Selected neurons were imported into the program NEURON and simulations run using the soma diameter of the neuron along with the actual dendritic tree morphology. A simplified model of one previously published was utilized with the following channels ionic currents: fast-sodium $\left(\mathrm{I}_{\mathrm{Na}}\right)$, persistent sodium $\left(\mathrm{I}_{\mathrm{NaP}}\right)$, fast-transient potassium $\left(\mathrm{I}_{\mathrm{A}}\right)$, potassium $\left(\mathrm{I}_{\mathrm{K}}\right)$, hyperpolarization-activated current $\left(\mathrm{I}_{\mathrm{h}}\right)$, and potassium leakage $\left(\mathrm{I}_{\text {leak }}\right)$ (Engel et al., 1999; Fietkiewicz et al., 2011; Purvis and Butera, 2005). The HodgkinHuxley formation for the membrane potential is given by the equation 


$$
\begin{aligned}
C_{m} \frac{d V}{d t}=I_{\text {leak }} & +I_{N a P}+I_{A}+I_{N a(\text { soma })}+I_{h(\text { soma })}+I_{K(\text { soma })}+I_{N a(\text { dendrite })}+I_{h(\text { dendrite })} \\
& +I_{K(\text { dendrite })}
\end{aligned}
$$

Where $\mathrm{V}$ is the membrane potential, $\mathrm{t}$ is time, and $\mathrm{C}_{\mathrm{m}}$ is the membrane capacitance. For an ionic current $I_{x}$, the current is expressed by the equation

$$
I_{x}=\bar{g}_{x} \cdot y_{x} \cdot\left(V-E_{x}\right)
$$

Where $\bar{g}_{x}$ is the maximum conductance, $y_{x}$ is the product of one or more activation or inactivation gating variables raised to integer powers, $\mathrm{V}$ is the membrane potential, and $E_{x}$ is the equilibrium potential. The equations for each $y_{x}$ were taken from the literature (Fietkiewicz et al., 2011; Purvis and Butera, 2005). The initial values for the parameters were taken from previous work (Fietkiewicz et al., 2011) and then iterations performed to adjust values until there was a match to published experimental data. For both soma and dendrites, the cytoplasmic resistance was $100 \mathrm{Ohm}-\mathrm{cm}$, the membrane capacitance was $0.25 \mathrm{pF} / \mathrm{cm}^{2}$, and the passive properties were $\mathrm{g}_{\mathrm{pas}}=0.00025 \mathrm{~S} / \mathrm{cm}^{2}$ with $\mathrm{E}_{\mathrm{pas}}=-70 \mathrm{mV}$, $\mathrm{g}_{\text {leak }}=0.000125 \mathrm{~S} / \mathrm{cm}^{2}$ with $\mathrm{E}_{\text {leak }}=-50 \mathrm{mV}, \mathrm{g}_{\mathrm{NaP}}=0.00003975 \mathrm{~S} / \mathrm{cm}^{2}$, and $\mathrm{g}_{\mathrm{A}}=0.0025 \mathrm{~S} / \mathrm{cm}^{2}$. For the soma $\mathrm{g}_{\mathrm{Na}(\mathrm{soma})}=0.0075 \mathrm{~S} / \mathrm{cm}^{2}$ with $\mathrm{E}_{\mathrm{Na}(\mathrm{soma})}=60 \mathrm{mV}, \mathrm{g}_{\mathrm{K}(\mathrm{soma})}=0.032 \mathrm{~S} / \mathrm{cm}^{2}$ with $\mathrm{E}_{\mathrm{K}(\mathrm{soma})}=-80 \mathrm{mV}$, and $\mathrm{g}_{\mathrm{h}(\mathrm{soma})}=0.00025 \mathrm{~S} / \mathrm{cm}^{2}$ with $\mathrm{E}_{\mathrm{h}(\mathrm{soma})}=-38.8 \mathrm{mV}$. For the dendrites $\mathrm{g}_{\mathrm{Na}(\text { dendrite })}=0.00075 \mathrm{~S} / \mathrm{cm}^{2}$ with $\mathrm{E}_{\mathrm{Na}(\text { dendrite })}=60 \mathrm{mV}, \mathrm{g}_{\mathrm{K}(\text { dendrite })}=0.0032 \mathrm{~S} / \mathrm{cm}^{2}$ with $\mathrm{E}_{\mathrm{K}(\text { dendrite })}=-80 \mathrm{mV}$, and $\mathrm{g}_{\mathrm{h}(\text { dendrite })}=0.000025 \mathrm{~S} / \mathrm{cm}^{2}$ with $\mathrm{E}_{\mathrm{h}(\text { dendrite })}=-38.8 \mathrm{mV}$.

Two different experiments were simulated with the model replicating previous electrophysiological data from genioglossal MNs (Nunez-Abades et al., 1993). We selected one experiment that yielded passive properties from current-voltage plots from 
current clamp. The experiment and our simulation injected currents from 0.1 to $0.6 \mathrm{nA}$ for P3 XII MNs and from 0.1 to $1 \mathrm{nA}$ for P10 and P17 both in increments of $0.1 \mathrm{nA}$. The other experiment generated single action potentials yielding active properties including the medium afterhyperpolarization (mAHP) potential (Nunez-Abades et al., 1993; Purvis and Butera, 2005). In the reported experiment the membrane was held at a fixed potential varying from $-50 \mathrm{mV}$ to $-85 \mathrm{mV}$ while a current was injected at a duration of $0.1 \mathrm{~ms}$. In our simulation we chose to set the $\mathrm{E}_{\mathrm{pas}}$ to values of $-50,-60,-70,-80$, and $-85 \mathrm{mV}$ to generate the five values reported in the experiment. The results of the simulation were compared with those previously obtained from the reported experiments (Nunez-Abades et al., 1993).

\section{Data Analysis}

Descriptive summary statistics of simulation results were generated for each of the three ages. Correlation coefficients and regression analysis was performed for the electrophysiological measures as a function of the morphology (volume or surface area). Nonparametric statistics were performed using the Kruskal-Wallis test and Dunn multiple comparisons to compare measured and simulation results. The data was fitted with the equation $Y=a \times X^{b}$, where ' $a$ ' is the intercept and ' $b$ ' is the exponent or slope of the curve. 


\section{Results}

\section{D Morphometrics and Electrophysiology}

Examples (average volume for either soma or dendrites) of 3D reconstructions and Sholl profiles for P3, P10, and P17 are shown in Figure 18. As can be seen by the Sholl profiles the morphology changes with increases in dendritic tree size and complexity. The total volume and surface area increased with age. The total volume increased by a factor of about 1.52 from P3 to P10 and 3.55 from P3 to P17 (Table 11). The total surface area increased by a factor of about 1.67 from P3 to P10 and 3.17 from P3 to P17 (Table 11). Statistically, only the P3 and P17 were significantly different for both volume and surface area. Power analysis suggested that approximately 75 P3 and P10 neurons each would be required for a power of 0.80. Examples of the simulation output from the current-voltage experiment for the P3, P10, and P17 XII MNs are shown in Figure 19. It was necessary to decrease the hyperpolarization-activated current $\left(\mathrm{I}_{\mathrm{h}}\right)$ by a factor of 10 for both soma and dendrites, increase membrane capacitance by a factor of 2 to match the P3 results with experimental results. With the decreased hyperpolarizationactivated current and increased membrane capacitance, the peak resistance did not change significantly, but the steady-state resistance did increase. Both peak and steady-state resistance decreased with age. From P3 to P17 the peak resistance decreased by $63 \%$ while the steady-state decreased by $71 \%$. For the single action potential (Figure 20) we observed decreasing width in the action potential with increasing age and the mAHP amplitude decreased with increasing membrane potential (Figure 20). There was no obvious statistically significant change in mAHP amplitude or duration with age (Table 
11), but power analysis suggested that for mAHP amplitude, a sample size of 75 for each age was required for a power $=0.8$.

\section{Electrophysiology as a Function of Morphology}

The peak resistance $\left(\mathrm{R}_{\text {peak }}\right)$ correlated with the total volume and surface area of the XII MNs (Figures 21A and 21B). The intercept of the curve for total volume versus $R_{\text {peak }}($ Figure 21A) was 2212 with a slope of $-0.38(R=0.5462, p<0.01)$ while the total volume surface area versus $R_{\text {peak }}$ (Figure 21B) had an intercept of 8227 with a slope of 0.53 (R-0.5831, p<0.01). Although the amplitude of the mAHP was not found to correlate $(\mathrm{R}=0.3375, \mathrm{p}>0.20$ for $\mathrm{P} 3$ and $\mathrm{R}=0.307, \mathrm{p}>0.20$ for $\mathrm{P} 10 / \mathrm{P} 17)$ with neuron morphology (Figure 21C), there was a correlation between $\mathrm{R}_{\text {peak }}$ and the amplitude of the mAHP (Figure 21D) only for P10 \& P17. For the P3 XII MNs the intercept for $\mathrm{R}_{\text {peak }}$ versus the amplitude of the mAHP was 1.43 with a slope of $0.13(\mathrm{R}=0.4058 . \mathrm{P}>0.10)$ and for P10/P17 the intercept was 1.15 with a slope of $0.29(\mathrm{R}=0.6796, \mathrm{p}<0.01)$. 

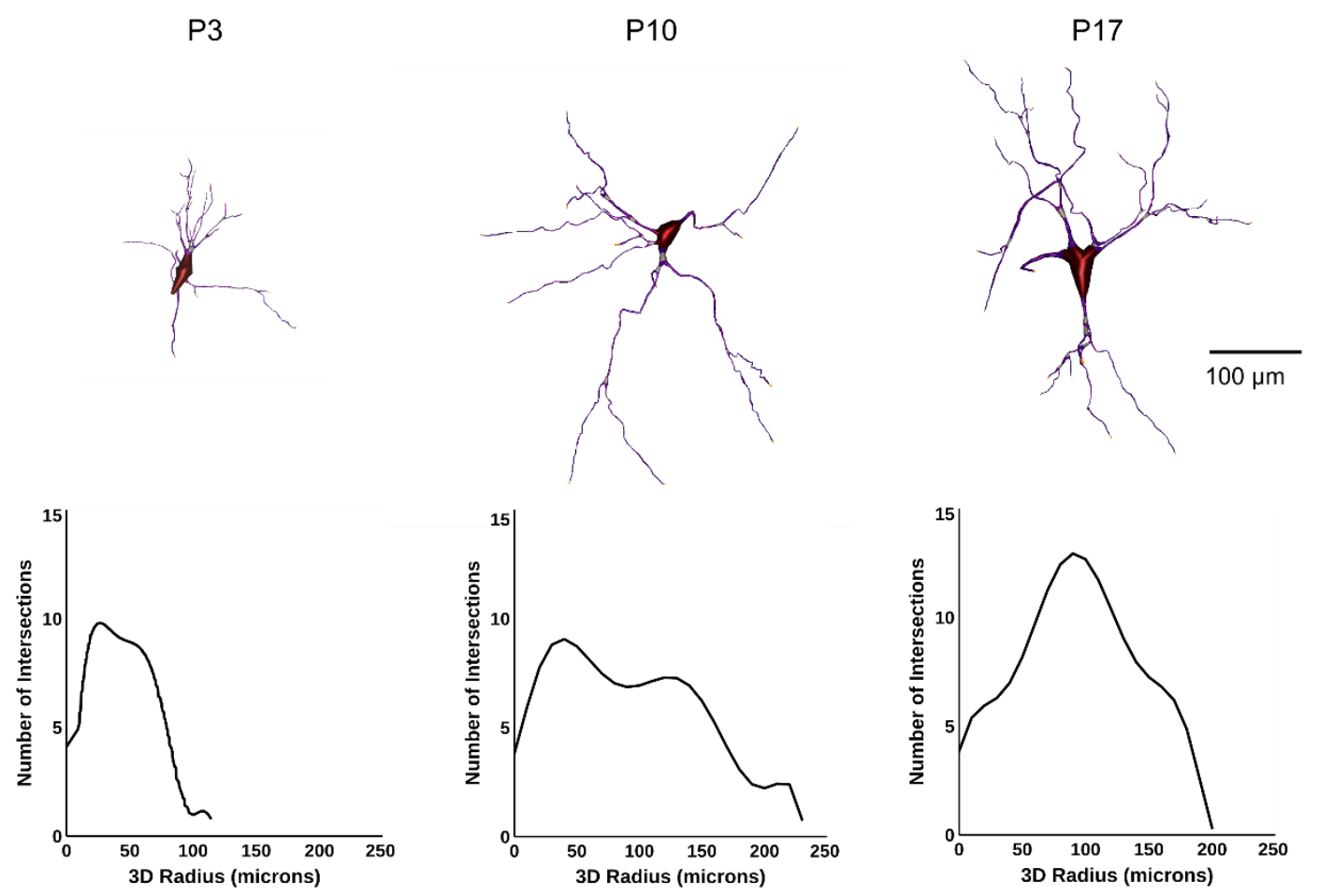

Figure 18. Morphology of hypoglossal motoneurons at P3, P10, and P17. The 3D reconstructions (top) and their associated Sholl profiles (bottom) are shown. 
Table 11. Summary statistics of morphological and biophysical properties from XII MNs. For each age the mean \pm standard deviation is given for each parameter along with the median shown in (). Shown are the morphometric parameters (volume and surface area), passive properties (peak and steady-state resistance), and the active properties (amplitude and duration of the medium afterhyperpolarization potential, mAHP).

\begin{tabular}{lccc}
\hline Parameter & P3 $(\mathbf{N}=\mathbf{1 0})$ & P10 $(\mathbf{N}=\mathbf{6})$ & P17 $(\mathbf{N}=\mathbf{6})$ \\
\hline Volume $\left(\mu \mathrm{m}^{3}\right)$ & $4766 \pm 4510$ & $7259 \pm 6104$ & $16904 \pm 6997$ \\
& $(3334)$ & $(5156)$ & $(17390)$ \\
Surface Area $\left(\mu \mathrm{m}^{2}\right)$ & $4258 \pm 3759$ & $7096 \pm 3856$ & $13510 \pm 3868$ \\
& $(3320)$ & $(7112)$ & $(12822)$ \\
Peak Resistance $(\mathrm{M} \Omega)$ & $143 \pm 101$ & $129 \pm 50$ & $53 \pm 36$ \\
Steady-State Resistance $(\mathrm{M} \Omega)$ & $(117)$ & $(126)$ & $(52)$ \\
& $(114)$ & $102 \pm 42$ & $41 \pm 26$ \\
mAHP amplitude $(\mathrm{mV})$ & $2.77 \pm 0.65$ & $4.61 \pm 1.21$ & $3.69 \pm 1.35$ \\
& $(2.57)$ & $(4.57)$ & $(3.70)$ \\
mAHP duration $(\mathrm{ms})$ & $173 \pm 37$ & $205 \pm 21$ & $184 \pm 34$ \\
& $(183)$ & $(211)$ & $(190)$ \\
\hline
\end{tabular}




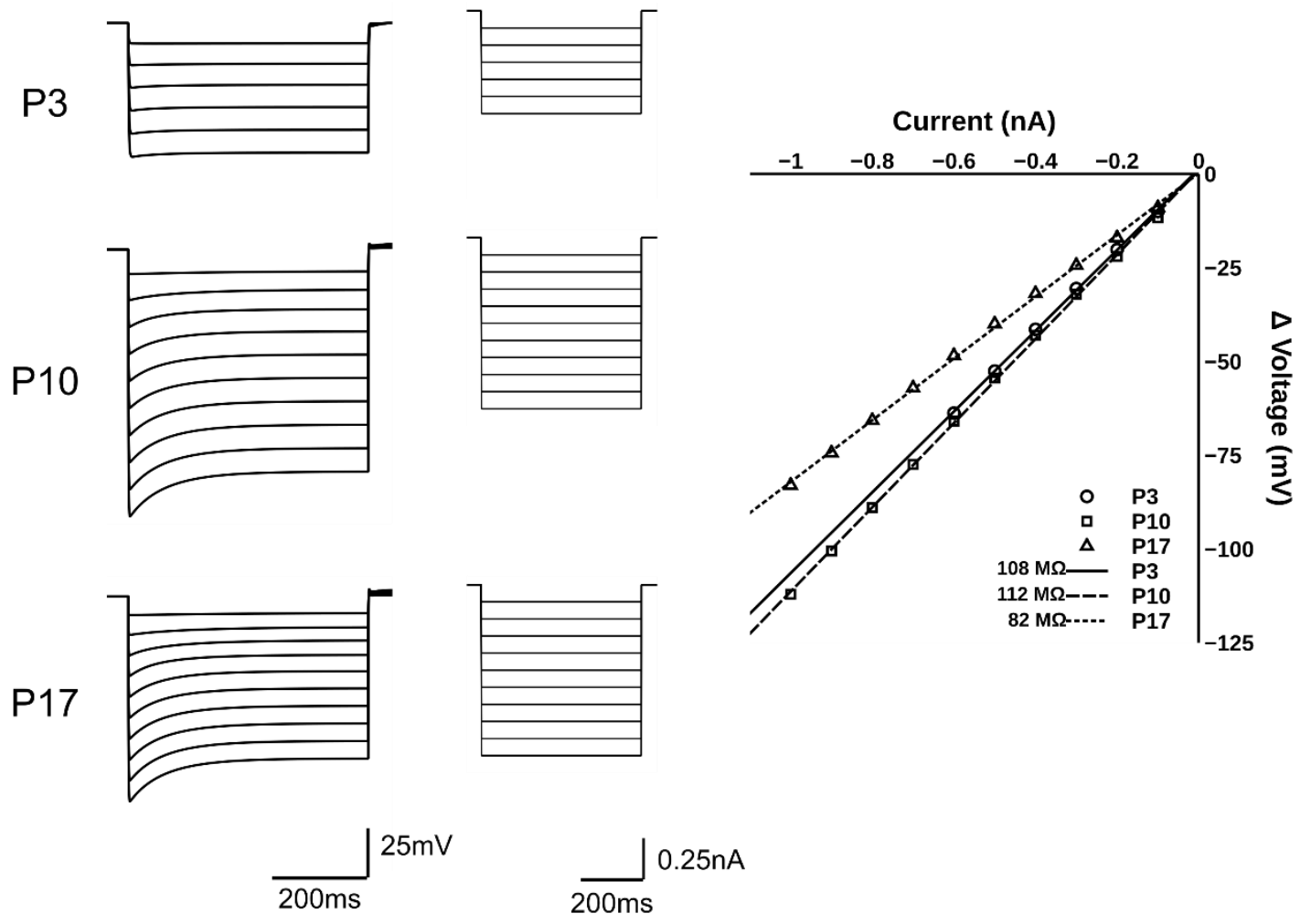

Figure 19. The I-V curves from P3, P10, and P17 XII MNs. On the left side are the voltage curves corresponding to the injected current (right side) and on the far right is the currentvoltage (I-V) curves for the three age groups showing the slopes (resistance). 


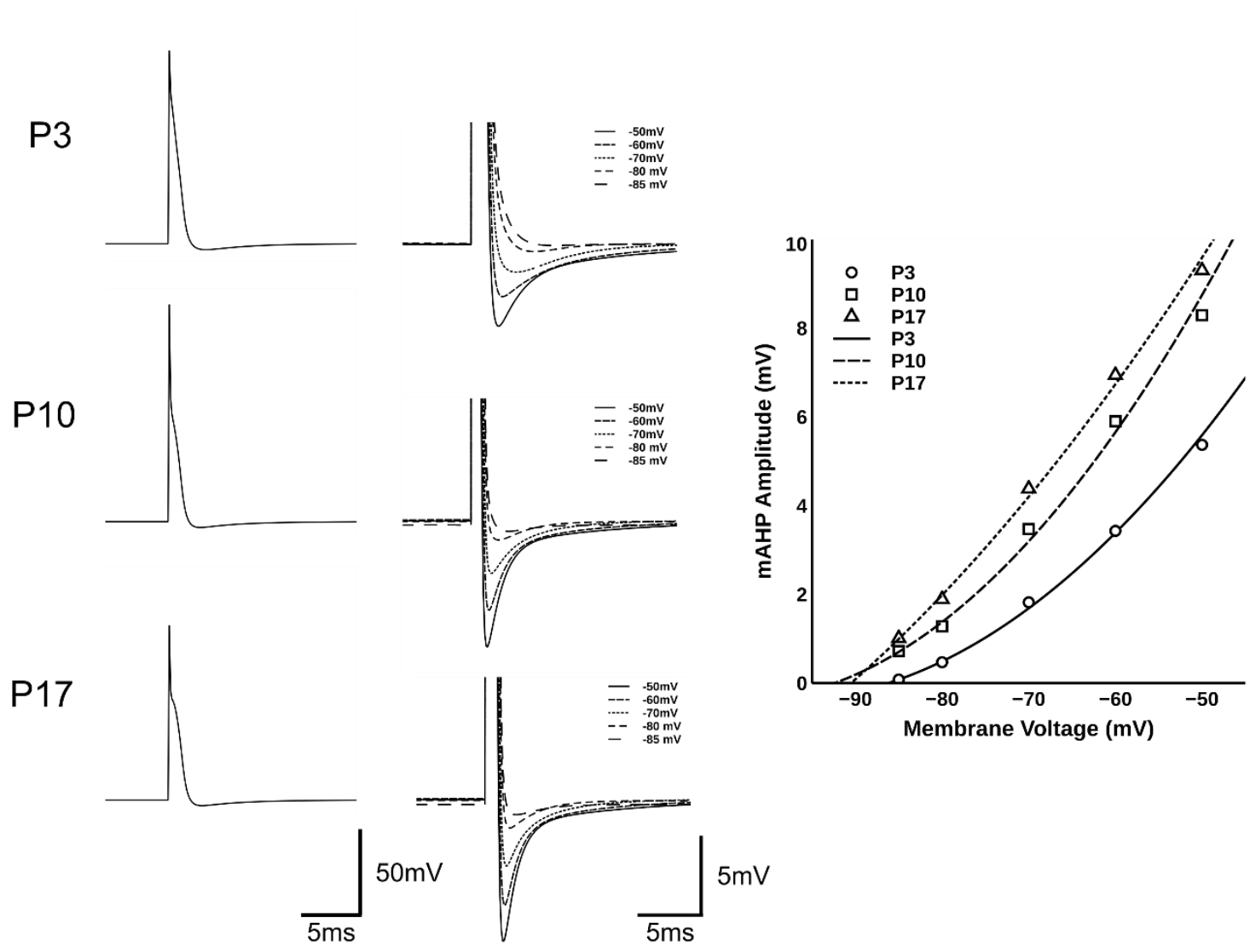

Figure 20. Action potentials and mAHP from P3, P10, and P17 XII MNs. On the left are the action potentials with expanded views (right) showing the mAHP potentials at each of the membrane potentials. On the far right is a graph of the membrane potential versus the mAHP amplitude for each of the three postnatal ages. 

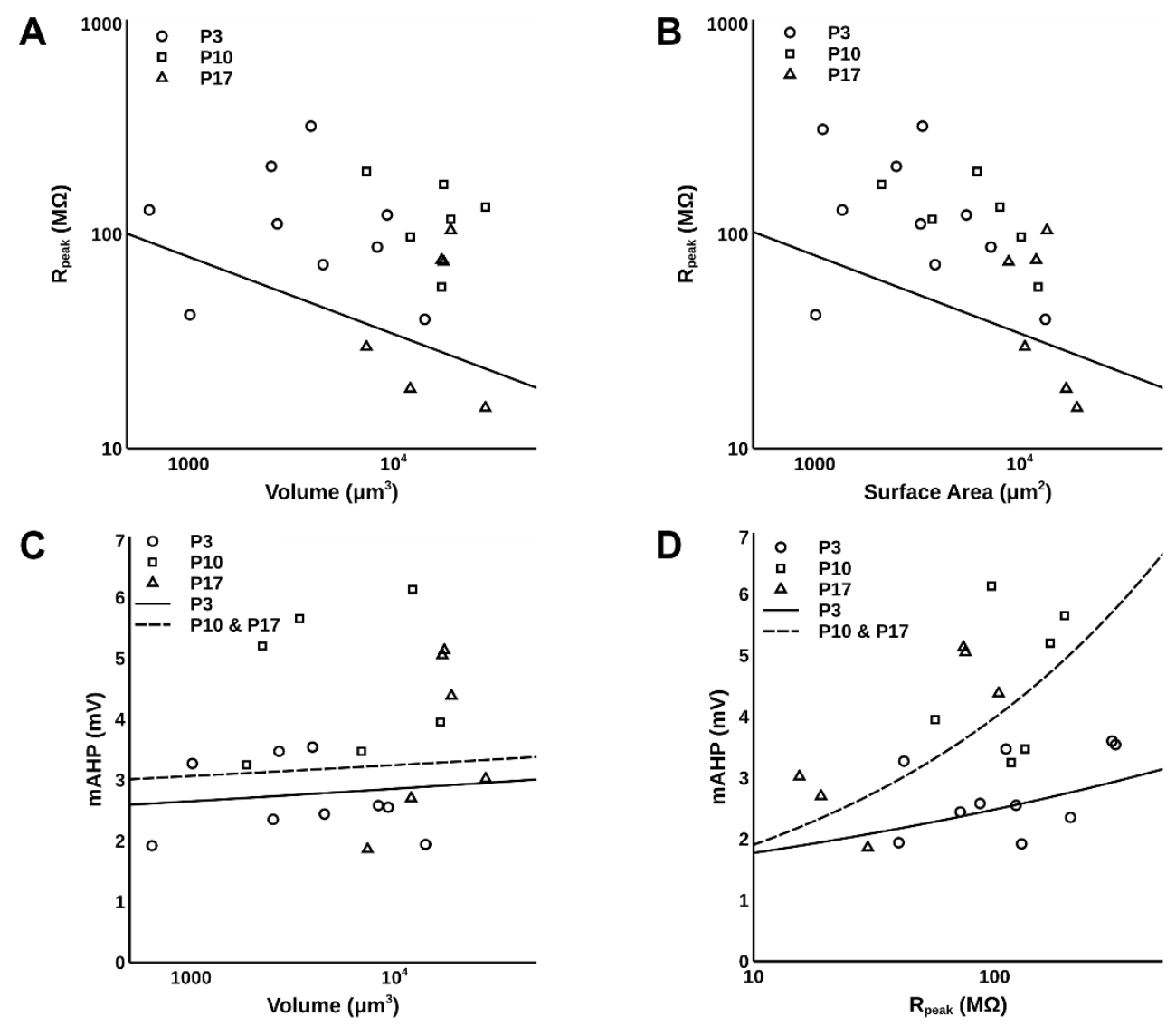

Figure 21. Regression analysis of passive and active biophysical properties of XII MNs as a function of morphology. Shown are a) total neuron volume versus peak resistance $\left(R_{\text {peak }}\right)$ with regression line for P3, P10, and P17 combined, b) total neuron surface area versus $\mathrm{R}_{\text {peak }}$ with regression line for P3, P10, and P17 combined, c) total neuron volume versus mAHP amplitude with regression for P3 only and regression for P10 and P17 combined,

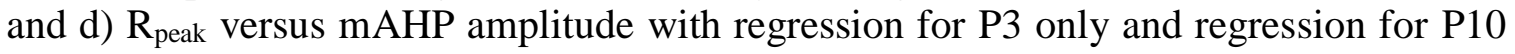
and P17 combined. All regression values are reported in the text. 


\section{Discussion}

In this study we have, for the first time, examined the potential relationship between the electrophysiological behavior and the morphology of XII MNs. We did not establish a direct relationship between the morphology of the neuron and amplitude of the mAHP, but we did show that mAHP amplitude is dependent on the neuron's passive properties, which suggests an indirect relationship between morphology and active properties. We simulated XII MN electrophysiology behavior based on 3D morphology measured from XII MNs in a rat pup model comparing theoretical passive and active electrophysiology properties to previously reported experimental results (Nunez-Abades et al., 1993). Although there are other studies of hypoglossal motoneuron electrophysiology (Bayliss et al., 1994; Lamanauskas and Nistri, 2008; Lape and Nistri, 2001; Powers and Binder, 2003), most studied specific ion currents. Simulating these studies could provide some insight into the ion currents, but do not provide information on morphology or differences in ion currents between soma and dendrites. Additionally, these studies did not examine the ages (P1-P2, P5-P6, P13-P15, and P19-P30) that Nunez-Abades et al. (1993) examined. The action potentials and mAHP amplitudes where similar to those in the literature (Nunez-Abades et al., 1993). To match the waveforms from published experimental results (Nunez-Abades et al., 1993) we had to alter the P3 XII MN parameters from those of the P10 and P17 XII MN parameters. We were able to demonstrate a relationship between neuron size (volume and surface area) and the passive properties showing decreased resistance (from I-V curves) with increased cell size. Because the resistance is mostly due to the passive biophysical properties there was little, if any differences between the P3 XII MNs and the P10/P17 XII MNs. With a 
2-fold increase in total volume there was a 20\% decrease in mAHP amplitude from P10 to P17. Since the simulation parameters were the same this change in mAHP could be due to the increase in total volume.

\section{Electrophysiology}

Although the model we used was a single (somal) compartment, we incorporated dendrites using the tools provided in NEURON (Fietkiewicz et al., 2011; Purvis and Butera, 2005). In our model we utilized different values of fast sodium and potassium conductance for soma and dendrites with the dendrites being 10-fold lower than the soma, as well as, the hyperpolarization-activated current being 100-fold lower for dendrites than for soma. A previous model, which included three dendrites with the soma, also used different values for conductance of specific channels (Engel et al., 1999). In this study, we found that the passive and active properties used for postnatal day 3 (P3) in our model did not match experimental results unless the values for the hyperpolarizationactivated current and membrane capacitance were different from twice the value of those that used for P10 and P17 neurons. Purvis and Butera (2005) have suggested that the hyperpolarization-activated current is lower in neonates based on differences in mAHP duration between adults and neonates with approximately a ten-fold decrease in neonates compared with adults. Experimental data on membrane properties and cell size suggest that the membrane capacitance can decrease by one-half from P1-P2 to P19-P30 (NunezAbades and Cameron, 1997) meaning that the value at $\mathrm{P} 3$ is not necessarily the same at $\mathrm{P} 10$ or P17. This may also be true for the P10 neuron since the membrane properties appear to change with age (Nunez-Abades and Cameron, 1997), and the mAHP changes 
with age related to the hyperpolarization-activated current (Berger et al., 1996; Cameron and Nunez-Abades, 2000; Purvis and Butera, 2005; Yoshimoto et al., 2015). One study has shown that the electrophysiological properties of P10 XII MNs differs from that of P17 XII MNs (Nguyen et al., 2004). As a result, the electrophysiological properties of the P10 XII MNs in this study may be different from experimental, but the previous study we used for comparison did not measure P10 XII MNs (Nunez-Abades et al., 1993) so this is, as of yet, unknown. Therefore, the changes we made to the P3 model are supported by prior experimental work.

\section{Electrophysiology as a Function of Morphology}

We found that the peak resistance correlated with both the total volume and total surface area of the neuron. This has been previously demonstrated experimentally (Nunez-Abades and Cameron, 1997); however, we have shown that the proportion of a subset of modeled ionic conductances are different between the P3 neurons and the P10/P17 neurons. The membrane resistance $\left(\mathrm{R}_{\mathrm{M}}\right)$ decreases with increasing age, while the amplitude of the mAHP changes little and there is some change in mAHP duration with age. Although the amplitude of the mAHP did not correlate with the volume or surface area of the neuron, we saw a relationship between mAHP amplitude and resistance correlating with cell size. Therefore, while morphology may not directly influence the active properties it certainly indirectly affects the excitability of the neuron via the changes we report in the cell's passive properties. Thus, our model provides a more rigorous foundation for understanding how the developmental changes in brainstem 
neurons can contribute to changes in excitability and reliability of neural activity in the brainstem respiratory neural network.

\section{Comparison with Previous Studies}

Although this study is the first to relate biophysical properties and morphology in an extensive range of developing brainstem neurons, there are theoretical models and experimental studies that have focused on XII MNs. Our model is an extension of two previous models for XII MNs that were single compartment Hodgkin-Huxley style models (Fietkiewicz et al., 2011; Purvis and Butera, 2005). One main difference in our study was the use of morphological data from measured XII MNs to simulated experiments from electrophysiological studies published by others (Nunez-Abades et al., 1993), then comparing our simulated results with the published experimental results. Although one other study modeled a soma with three non-branching dendrites (Engel et al., 1999), our study used neurons of different sizes, dendrite lengths, and degrees of branching. An electrophysiology study on rat vibrissa motoneurons assessed measured active and passive membrane properties as well as measuring neuron morphology (Nguyen et al., 2004). They showed differences in branch length and membrane impedance between P4-P5 and P15-P23 neurons but did not show correlations between the membrane properties and neuron morphology.

\section{Limitations of Model}

Our model was limited by gaps in our knowledge concerning the range of ionic conductances expressed and present in the membrane of XII MNs, as well as, differences 
in channel density on the dendrite as compared with the soma and those changes occurring across the time course of early development. Other studies have established the location of some voltage-gated ion channels (Vacher et al., 2008), but little is known about which ion channels are expressed where for XII MNs. There are many studies of voltage-gated ion channel function (Ekberg and Adams, 2006; Jan and Jan, 2012; Johnston et al., 2010; Yu and Catterall, 2003) and XII MNs specifically (Bayliss et al., 1994; Lape and Nistri, 2001; Powers et al., 1999; Powers and Binder, 2003), but there is no comprehensive information about ion channel relative densities on soma and dendrites in XII MNs. One study found that ion channel expression in the rat ventral respiratory column varies between adult and neonates with a subset of sodium, potassium, and calcium channels having a six-fold or more increase in adults when compared with neonates (González-Castillo et al., 2017). However, in our study we found that the surface area in P17 neurons was about 3-fold greater compared with P3 neurons, suggesting that the density may have about a 2 -fold or more increase in our models. The different sub-types of voltage-gated ion channels and which are present on the somata and dendrites of XII MNs is another factor having a profound effect on electrophysiological behavior. Beckh and colleagues (1989) showed that from P1 to about P10 one sub-type of sodium channel III $\left(\mathrm{Na}_{\mathrm{v}} 1.3\right)$ is expressed on neurons while a different sub-type sodium channel I $\left(\mathrm{Na}_{\mathrm{v}} 1.1\right)$ is expressed from P10 through adults (Beckh et al., 1989). These two sodium channel sub-types $\left(\mathrm{Na}_{\mathrm{v}} 1.1\right.$ and $\left.\mathrm{Na}_{\mathrm{v}} 1.3\right)$ exhibit different activation inactivation, and deactivation kinetics (Ekberg and Adams, 2006; Vacher et al., 2008). Although there are studies of expression of various channels (González-Castillo et al., 2017; Beckh et al., 1989), most are for the whole brain and very 
few, if any studies of channel distribution on neurons are available for brainstem neurons and less for XII MNs.

\section{Future Directions}

In this study we have provided support for our hypothesis that morphological changes may have a direct or indirect role in changes of the biophysical properties and behavior of XII MNs. We also established support for changes in ion currents with age that were necessary for us to match our simulation results with previous experimental results. Therefore, both morphology and ion currents seem to be important in the biophysical behavior of XII MNs. However, major challenges in this study included our assumptions on channel location and density in the soma and dendrites. Studies employing immunohistochemistry to stain various channels, allowing quantification of numbers and location/density for XII MNs would aid in model development (Bocksteins et al., 2012) and these studies would be a fruitful avenue for future investigation. Although experimental electrophysiology on XII MNs using neurobiotin/biocytin fills have provided morphology in XII MNs, (Nguyen et al., 2004; Nunez-Abades and Cameron, 1997), there are few studies incorporating both electrophysiology and morphology for the same set of neurons at multiple ages. Combining this electrophysiology and morphology with modeling based on the morphology for direct comparison of modeling results with experimental results should provide detailed information about the relationship between morphology and biophysical properties in vitro. This information should also provide a better understanding of electrophysiological 
behavior as more detailed and extensive computational models are developed for motor systems as well as higher CNS neural networks.

\section{Conclusions}

The results from this study suggests that the passive properties (resistance and capacitance) correlate with the deverlopmental morphology changes in hypoglossal neurons. Although the active properties were not found to correlate as significantly with morphology, particularly the amplitude of mAHP, more work must be done to address this feature of XII MN modeling. Our modeling results and experimental data reported in published literature suggest that the ion currents are changing with age—resulting in changes of the passive and active properties. However, very few if any studies have thoroughly studied the electrophysiology of developing neurons in functional motor networks.

\section{Acknowledgements}

Imaging was performed in the LLUSM Advanced Imaging and Microscopy Core with support from National Science Foundation grant MRI-DBI 0923559 and Loma Linda University School of Medicine. The authors thank Johnny Figueroa, $\mathrm{PhD}$ for assistance and use of the Keyence microscope. 


\section{Literature Cited}

Bayliss, D.A., Viana, F., Bellingham, M.C., Berger, A.J., 1994. Characteristics and postnatal development of a hyperpolarization-activated inward current in rat hypoglossal motoneurons in vitro. J Neurophysiol 71, 119-28.

Beckh, S., Noda, M., Lubbert, H., Numa, S., 1989. Differential regulation of three sodium channel messenger RNAs in the rat central nervous system during development. EMBO J 8, 3611-6.

Berger, A.J., Bayliss, D.A., Viana, F., 1996. Development of hypoglossal motoneurons. J. Appl. Physiol. 81, 1039-1048.

Bocksteins, E., Shepherd, A.J., Mohapatra, D.P., Snyders, D.J., 2012. Immunostaining of Voltage-Gated Ion Channels in Cell Lines and Neurons - Key Concepts and Potential Pitfalls, in: Dehghani, H. (Ed.), Applications of Immunocytochemistry. InTech.

Boone, T.B., Aldes, L.D., 1984. The ultrastructure of two distinct neuron populations in the hypoglossal nucleus of the rat. Exp Brain Res 54, 321-6.

Cameron, W.E., Nunez-Abades, P.A., 2000. Physiological changes accompanying anatomical remodeling of mammalian motoneurons during postnatal development. Brain Res Bull 53, 523-7.

Carrascal, L., Nieto-Gonzalez, J.L., Cameron, W.E., Torres, B., Nunez-Abades, P.A., 2005. Changes during the postnatal development in physiological and anatomical characteristics of rat motoneurons studied in vitro. Brain Res Brain Res Rev 49, 377-87. https://doi.org/10.1016/j.brainresrev.2005.02.003

Cooper, M.H., 1981. Neurons of the hypoglossal nucleus of the rat. Otolaryngol Head Neck Surg 89, 10-5. https://doi.org/10.1177/019459988108900103

Das, G., Reuhl, K., Zhou, R., 2013. The Golgi-Cox method. Methods Mol Biol 1018, 313-21. https://doi.org/10.1007/978-1-62703-444-9_29

Ekberg, J., Adams, D.J., 2006. Neuronal voltage-gated sodium channel subtypes: key roles in inflammatory and neuropathic pain. Int J Biochem Cell Biol 38, 2005-10. https://doi.org/10.1016/j.biocel.2006.06.008

Engel, J., Schultens, H.A., Schild, D., 1999. Small conductance potassium channels cause an activity-dependent spike frequency adaptation and make the transfer function of neurons logarithmic. Biophys J 76, 1310-9. https://doi.org/10.1016/S00063495(99)77293-0

Feldman, J.L., Del Negro, C.A., 2006. Looking for inspiration: new perspectives on respiratory rhythm. Nat. Rev. Neurosci. 7, 232-242.

https://doi.org/10.1038/nrn1871 
Feldman, J.L., Del Negro, C.A., Gray, P.A., 2013. Understanding the rhythm of breathing: so near, yet so far. Annu. Rev. Physiol. 75, 423-452.

https://doi.org/10.1146/annurev-physiol-040510-130049

Feldman, J.L., Smith, J.C., 1995. Neural control of respiratory pattern in mammals: an overview, in: Dempsey, J.A., Pack, A.I. (Eds.), Regulation of Breathing. MarcelDekker, New York, N.Y., pp. 39-69.

Ferrante, M., Migliore, M., \& Ascoli, G. A., 2013. Functional impact of dendritic branchpoint morphology. J Neurosci, 33(5), 2156-2165. doi:10.1523/JNEUROSCI.3495-12.2013

Fietkiewicz, C., Loparo, K.A., Wilson, C.G., 2011. Drive latencies in hypoglossal motoneurons indicate developmental change in the brainstem respiratory network. J. Neural Eng. 8. https://doi.org/10.1088/1741-2560/8/6/065011

Fukunishi, Y., Nagase, Y., Yoshida, A., Moritani, M., Honma, S., Hirose, Y., Shigenaga, Y., 1999. Quantitative analysis of the dendritic architectures of cat hypoglossal motoneurons stained intracellularly with horseradish peroxidase. J Comp Neurol 405, 345-58.

Gibb, R., Kolb, B., 1998. A method for vibratome sectioning of Golgi-Cox stained whole rat brain. J Neurosci Methods 79, 1-4.

González-Castillo, C., Muñoz-Ortiz, E., Guzmán-Brambila, C., Rojas-Mayorquín, A.E., Beltran-Parrazal, L., Ortuño-Sahagún, D., Morgado-Valle, C., 2017. Differential Expression of Ion Channels in Adult and Neonatal Rat Ventral Respiratory Column. J. Mol. Neurosci. https://doi.org/10.1007/s12031-017-1001-2

Jan, L.Y., Jan, Y.N., 2012. Voltage-gated potassium channels and the diversity of electrical signalling. J Physiol 590, 2591-9. https://doi.org/10.1113/jphysiol.2011.224212

Johnston, J., Forsythe, I.D., Kopp-Scheinpflug, C., 2010. Going native: voltage-gated potassium channels controlling neuronal excitability. J Physiol 588, 3187-200. https://doi.org/10.1113/jphysiol.2010.191973

Kanjhan, R., Fogarty, M.J., Noakes, P.G., Bellingham, M.C., 2016. Developmental changes in the morphology of mouse hypoglossal motor neurons. Brain Struct. Funct. 221, 3755-3786. https://doi.org/10.1007/s00429-015-1130-8

Khazipov, R., Zaynutdinova, D., Ogievetsky, E., Valeeva, G., Mitrukhina, O., Manent, J.B., Represa, A., 2015. Atlas of the Postnatal Rat Brain in Stereotaxic Coordinates. Front. Neuroanat. 9. https://doi.org/10.3389/fnana.2015.00161

Koizumi, H., Wilson, C.G., Wong, S., Yamanishi, T., Koshiya, N., Smith, J.C., 2008. Functional Imaging, Spatial Reconstruction, and Biophysical Analysis of a Respiratory Motor Circuit Isolated In Vitro. J. Neurosci. 28, 2353-2365. 
Komendantov, A.O., Ascoli, G.A., 2009. Dendritic excitability and neuronal morphology as determinants of synaptic efficacy. J Neurophysiol 101, 1847-66.

Lamanauskas, N., Nistri, A., 2008. Riluzole blocks persistent $\mathrm{Na}+$ and $\mathrm{Ca} 2+$ currents and modulates release of glutamate via presynaptic NMDA receptors on neonatal rat hypoglossal motoneurons in vitro. Eur J Neurosci 27, 2501-14.

Lape, R., Nistri, A., 2001. Characteristics of fast $\mathrm{Na}(+)$ current of hypoglossal motoneurons in a rat brainstem slice preparation. Eur J Neurosci 13, 763-72.

Lindsey, B.G., Rybak, I.A., Smith, J.C., 2012. Computational models and emergent properties of respiratory neural networks. Compr Physiol 2, 1619-70. https://doi.org/10.1002/cphy.c110016

Liu, Q., Lowry, T.F., Wong-Riley, M.T.T., 2006. Postnatal changes in ventilation during normoxia and acute hypoxia in the rat: implication for a sensitive period. J Physiol 577, 957-70.

Mortola, J.P., 1984. Breathing pattern in newborns. J Appl Physiol Respir Env. Exerc Physiol 56, 1533-40.

McClung, J.R., Goldberg, S.J., 1999. Organization of motoneurons in the dorsal hypoglossal nucleus that innervate the retrusor muscles of the tongue in the rat. Anat Rec 254, 222-30.

Myatt, D.R., Hadlington, T., Ascoli, G.A., Nasuto, S.J., 2012. Neuromantic - from semimanual to semi-automatic reconstruction of neuron morphology. Front Neuroinform 6, 4. https://doi.org/10.3389/fninf.2012.00004

Nguyen, Q.T., Wessel, R., Kleinfeld, D., 2004. Developmental regulation of active and passive membrane properties in rat vibrissa motoneurones. J Physiol 556, 203-19. https://doi.org/10.1113/jphysiol.2003.060087

Nunez-Abades, P.A., Cameron, W.E., 1997. Relationship between membrane properties and cell size of developing rat genioglossal motoneurons studied in vitro. Neurosci Lett 223, 41-4.

Nunez-Abades, P.A., Cameron, W.E., 1995. Morphology of developing rat genioglossal motoneurons studied in vitro: relative changes in diameter and surface area of somata and dendrites. J Comp Neurol 353, 129-42. https://doi.org/10.1002/cne.903530112

Nunez-Abades, P.A., He, F., Barrionuevo, G., Cameron, W.E., 1994. Morphology of developing rat genioglossal motoneurons studied in vitro: changes in length, branching pattern, and spatial distribution of dendrites. J Comp Neurol 339, 40120. https://doi.org/10.1002/cne.903390308 
Nunez-Abades, P.A., Spielmann, J.M., Barrionuevo, G., Cameron, W.E., 1993. In vitro electrophysiology of developing genioglossal motoneurons in the rat. $\mathrm{J}$ Neurophysiol 70, 1401-11.

Powers, R.K., Binder, M.D., 2003. Persistent sodium and calcium currents in rat hypoglossal motoneurons. J Neurophysiol 89, 615-24. https://doi.org/10.1152/jn.00241.2002

Powers, R.K., Sawczuk, A., Musick, J.R., Binder, M.D., 1999. Multiple mechanisms of spike-frequency adaptation in motoneurones. J Physiol Paris 93, 101-14.

Preibisch, S., Saalfeld, S., Tomancak, P., 2009. Globally optimal stitching of tiled 3D microscopic image acquisitions. Bioinformatics 25, 1463-1465. https://doi.org/10.1093/bioinformatics/btp184

Purvis, L.K., Butera, R.J., 2005. Ionic Current Model of a Hypoglossal Motoneuron. J. Neurophysiol. 93, 723-733.

Smith, J.C., Butera, R.J., Koshiya, N., Negro, C.D., Wilson, C.G., Johnson, S.M., 2000. Respiratory rhythm generation in neonatal and adult mammals: the hybrid pacemaker-network model. Respir. Physiol. 122, 131-147.

Takasu, N., Hashimoto, P.H., 1988. Morphological identification of an interneuron in the hypoglossal nucleus of the rat: a combined Golgi-electron microscopic study. $\mathrm{J}$ Comp Neurol 271, 461-71. https://doi.org/10.1002/cne.902710313

Vacher, H., Mohapatra, D.P., Trimmer, J.S., 2008. Localization and targeting of voltagedependent ion channels in mammalian central neurons. Physiol Rev 88, 1407-47. https://doi.org/10.1152/physrev.00002.2008

Yoshimoto, R., Iwasaki, S., Takago, H., Nakajima, T., Sahara, Y., Kitamura, K., 2015. Developmental increase in hyperpolarization-activated current regulates intrinsic firing properties in rat vestibular ganglion cells. Neuroscience 284, 632-42. https://doi.org/10.1016/j.neuroscience.2014.10.034

Yu, F.H., Catterall, W.A., 2003. Overview of the voltage-gated sodium channel family. Genome Biol 4. 


\section{CHAPTER FOUR}

\section{DISCUSSION}

Brainstem regions including the nucleus tractus solitarii, ventral respiratory column, Bötzinger Complex, pre-Bötzinger Complex, and hypoglossal motor nucleus are necessary for the generation and transmission of breathing rhythm and modification of breathing pattern in response to sensory input (Feldman et al., 2013; Feldman and Del Negro, 2006; Lindsey et al., 2012; Wong-Riley and Liu, 2005). This respiratory network plays a key role in breathing pattern and control (Lindsey et al., 2012; Liu et al., 2006; Mortola, 1984). This neural network behavior can be influenced by the morphology of neurons, and connectivity can be altered due to changes in neurotransmitter release and receptor up- and down-regulation (Ferrante et al., 2013; Komendantov and Ascoli, 2009). Although not fully investigated, it has been suggested that morphology, neuron electrophysiology, connectivity, and neurotransmitter expression changes during development (Carrascal et al., 2005; Liu et al., 2009; Nunez-Abades et al., 1993; WongRiley and Liu, 2005). Therefore, understanding how the breathing neural network is remodeled during early life is key to elucidating the synergy between morphology and the transmission of breathing rhythm in the perinatal period.

\section{Developmental Morphology of Hypoglossal Motoneurons}

In this work I have shown that the XII MN soma increases size with increasing age, but that the shape changes very little, if any with increasing age. This supports a recent study on XII MNs in mice (Refik Kanjhan et al., 2016) and suggest that the soma has a gradual, continuous growth during the first three weeks post birth. However, the 
dendritic tree increases in both size and complexity with increasing age. More importantly, I have shown that there are at least three development windows: P1-P5, P7$\mathrm{P} 12$, and P13-P21. One of the main features of these three windows is the increase in the number of primary branches during the $\mathrm{P} 7-\mathrm{P} 12$ period while during the $\mathrm{P} 13-\mathrm{P} 21$ period there is only an increase in branching. This supports our hypothesis of the sensitive period in that the increase in primary branches specifically during this P7-P12 window represents a change which allows for more extensive branching influencing the surface area available for ion channels and receptors. Another result supporting my hypothesis is that the data could not be fitted to a single distribution, to a finite mixture model of two normal distributions with the parameters being age-window dependent. For example, the radius at which the maximum number of intersections occurred did not have the same distribution parameters at $\mathrm{P} 7-\mathrm{P} 12$ as at $\mathrm{P} 1-\mathrm{P} 5$. At $\mathrm{P} 13-\mathrm{P} 21$ the data was more represented as a single distribution, but the P7-P12 seems to represent a transition between the P1-P5 and P13-P21. Therefore, there does appear to be changes that are consistent with a morphological "sensitive period" or a transitional window between the first and third weeks post-birth.

Except for the soma shape which did not change significantly with age, the size of the soma, dendritic tree size, and complexity of dendritic trees all increased with increasing age. The finite mixture model of two normal distributions was not consistent for the three developmental windows (except for aspect ratio and form factor) suggesting that soma size and dendrite development is not necessarily the same for all neurons at a specific age. Changes in soma size relates in part to the metabolic capacity and dendritic tree size and complexity relates partially to connectivity. If the dendritic tree lacks 
sufficient surface area, then the number of synaptic contacts and ion channels is limited which may impact function. In the hypoglossal nucleus this could limit the drive and control of the tongue and upper airway potentially having a negative impact on upper airway function.

\section{Developmental Electrophysiology of Hypoglossal Motoneurons}

The principle goal of modeling is to provide some theoretical understanding of a phenomena and insight into testable hypotheses which can be tested experimentally. Computer simulation, done correctly, can simulate several experiments in a shorter time than actual experiments generating questions that can be addressed experimentally. Modelling is simply an adjunct to experimental work which should complement and enhance experimental data and results. I have shown that both passive and active properties change with increasing age. Important to the electrophysiology, I have shown that the passive properties correlate with morphology. Another important finding was that for the P3 XII MNs it was necessary in the model to double the membrane capacitance and decrease the hyperpolarization-activated current by ten to replicate the passive and active properties measured experimentally. This would suggest that there are developmental changes in at least some of the ionic currents such as the hyperpolarization-activated current as well as the membrane capacitance with age. Both of these have been suggested in previous studies (Nunez-Abades and Cameron, 1997; Purvis and Butera, 2005). However, very little is known about any changes in other ion currents such as the various types and sub-types of sodium and potassium currents with age. Further study of the ionic currents at individual ages might provide more 
understanding of the functional changes in neurons. Especially if there are any changes during the sensitive period reflecting transitions in how the ion currents contribute to the neuron's electrophysiology could provide clues into other functional changes.

\section{Limitations}

There are several limitations from each part of this work. The Golgi-Cox staining method is neuron selective, but what neurons are selectively stain and why are not apparent. The 3D reconstructions are from image stacks with each image take at intervals of $2 \mu \mathrm{m}$. At a magnification of 40x the resolution in the $\mathrm{x}$ and $\mathrm{y}$ direction is about \pm 0.1 $\mu \mathrm{m}$. Therefore, the reconstructions do not represent true 3D models since an orthogonal view is not acquired and the resolution in the z-direction is about $\pm 1 \mu \mathrm{m}$. This translates to lack of accuracy and loss of information on dendrites extending from the bottom and top of the soma. Neuromantic and other programs for 3D reconstruction generally assume cylinder and conical shapes which may not completely represent actual morphologies of the neuron soma or dendrite.

The simulation environment NEURON assumes a spherical soma consisting of a single compartment, but this does not reflect the actual 3D morphology of XII MNs. Many of the parameters for the model such as the ionic current densities for XII MN soma and dendrites are not known. Previous electrophysiological studies cannot distinguish between soma and dendritic channels. Studies investigating the expression of voltage-gated channels usually process and examine the whole brain and not specific regions. There are some studies on localization for voltage-gated channels, however these studies are for neurons in general and not specific to XII MNs. I did not include calcium 
channels nor the small-conductance calcium-activated potassium (SK) channel which are less important to single action potentials and more important to a train of action potentials. Therefore, an improvement to the present work would be to incorporate these ionic currents into the model for passive, single action potentials, and action potential trains.

\section{Future Studies}

One obvious extension of this work would be the 3D reconstructions and morphometric analysis of the XII MNs. This is presently being pursued for all 460 XII MNs reported in this study for later publication. Measures such as branch order, dendrite length and diameter at each branch order, and other 3D morphometrics are being obtained to generate dendrograms and graphs to summarize these geometric quantities.

Some of the questions about changes in ion currents with age resulting from the electrophysiology simulations in this work could possibly be addressed by two strategies. Since there are antibodies for some types and sub-types of voltage-gated channels immunohistochemical techniques could be used to estimate the densities of these channels on soma and dendrites of XII MNs (Bocksteins et al., 2012). Although actual densities would be of benefit, estimates of the ratio of ion channel densities between soma and dendrites could provide a constraint in current densities for the simulation models. By increasing the number of known parameters, the number of solutions is constrained thereby providing a convergence of model parameters such that simulations optimally match experimental results. Another approach would be to use pharmacological techniques to block specific ion currents. Pharmacological agents such 
as TTX can be used to block sodium channels while agents such as minocycline can block the hyperpolarization-activated current (Bayliss et al., 1994; Berger et al., 1996; Carrascal et al., 2005). Reducing the number of ionic currents in the experiment would also reduce the number of parameters in the simulation model reducing the number of iterations to match simulated and experimental results. By matching the simulated and experimental results with different ionic currents being blocked only specific ionic currents combinations would be acting for each scenario allowing for stepwise iteration of the parameters. Both approaches are limited due to lack of antibodies for all channels and methodological limits for immunohistochemistry, and the limitation of pharmacological agents targeting ionic currents. Therefore, by combining immunohistochemistry and pharmacological approaches most of the ionic current associated parameters could be worked out in the simulation model based on experimental data.

Probably the most important direction for this work stems from the finding that morphology has an indirect influence on active electrophysiological properties. By determining the corresponding morphology of neurons with a specific electrophysiological behavior a better understanding of the electrophysiology could be obtained. One experiment to address this would be to perform electrophysiology on XII motoneurons in rat pups from the different developmental windows. After the experiment is completed fill the neuron with neurobiotin processing for either bright-field or fluorescence imaging, acquire image stacks, and perform 3D reconstructions obtaining 3D morphology. These 3D geometrical models could then be imported into NEURON or another simulation program where the experiment would be replicated, and simulated 
results compared with the experimental results. By having the morphology to normalize the comparison between simulated and experimental results any influence or variance added by the morphology is accounted for allowing for better iteration of the ion currents. However, it would be necessary to obtain a sufficient variance in the morphology to be able to ascertain the relationship between morphology and electrophysiological properties. Therefore, in this experiment both a suitable electrophysiological model for simulation, and sufficient sample size and morphological variance is required for curve fitting.

Other experiments that would expand the findings of my work would be to repeat this work with different animals. This comparative morphology-electrophysiology could provide a broader understanding of XII MNs exploring conserved and non-conserved aspects of XII MNs. Differences in respiration and responses to conditions such as hypoxia and hypercapnia between various strains of rats has been reported (Golder et al., 2005; Strohl et al., 1997; Yang et al., 1999). Therefore, the study of other rat strains, such as Brown Norway, Wistar, or Fischer compared to the Sprague-Dawley rats used in this study would provide more depth in our understanding of XII MNs and development. Establishing the pattern of maturation of XII MNs in two or more rat strains such as Brown Norway or Wistar could provide a deeper understanding of neuron development from the similarity and differences particularly in morphology, electrophysiology, and their relationship. Since the rat pup model is one of premature birth since several systems such as thermoregulation and respiration are not fully developed at birth another extension of this would be to investigate XII MN development in full-term animal models such as guinea pigs. Rats differ from other rodents such as mice (Mortola, 1991, 
1984; Mortola and Lanthier, 2004), so characterization of the XII MN maturation in these species compared to rats would provide a wider understanding of XII MN morphology with age. Understanding the similarities and differences in animal models of full-term and preterm births could provide information directed to problems associated with preterm births.

Finally, the effects of conditions such as perinatal nicotine exposure or maternal inflammation on the developmental maturation of XII MNs in a rat pup model could be more precisely determined since there is now a baseline for normal maturation. In conjunction with the previously mentioned studies a suitable simulation model for electrophysiological behavior for XII MNs and its relationship with morphology can be established. This foundation for understanding could allow for improved understanding of both the morphological and electrophysiological changes due to adverse conditions such as nicotine exposure or inflammation leading to better interpretation of short- and long-term implications of these conditions. One such implication from this present work is the differential morphology (finite mixture model results). One hypothesis for perinatal nicotine exposure or maternal inflammation could be the lack of the differential morphology. If certain morphological parameters which are non-normally distributed are found to be normally distributed in the perinatal nicotine exposure or maternal inflammation animals, then this could have implications even if there are no statistical differences due to sample size or variance. Having a complete and detailed understanding of the natural maturation of XII MNs could provide the foundation for adequate interpretation of perinatal, maternal, and even postnatal conditions such as nicotine and inflammation. 


\section{REFERENCES}

Aldes, L.D., 1995. Subcompartmental organization of the ventral (protrusor) compartment in the hypoglossal nucleus of the rat. J. Comp. Neurol. 353, 89-108.

Ascoli, G.A., Brown, K.M., Calixto, E., Card, J.P., Galvan, E.J., Perez-Rosello, T., Barrionuevo, G., 2009. Quantitative morphometry of electrophysiologically identified CA3b interneurons reveals robust local geometry and distinct cell classes. J Comp Neurol 515, 677-95. https://doi.org/10.1002/cne.22082

Bayliss, D.A., Viana, F., Bellingham, M.C., Berger, A.J., 1994. Characteristics and postnatal development of a hyperpolarization-activated inward current in rat hypoglossal motoneurons in vitro. J Neurophysiol 71, 119-28.

Beckh, S., Noda, M., Lubbert, H., Numa, S., 1989. Differential regulation of three sodium channel messenger RNAs in the rat central nervous system during development. EMBO J 8, 3611-6.

Berger, A.J., Bayliss, D.A., Viana, F., 1996. Development of hypoglossal motoneurons. J. Appl. Physiol. 81, 1039-1048.

Bocksteins, E., Shepherd, A.J., Mohapatra, D.P., Snyders, D.J., 2012. Immunostaining of Voltage-Gated Ion Channels in Cell Lines and Neurons -- KeyConcepts and Potential Pitfalls, in: Dehghani, H. (Ed.), Applications of Immunocytochemistry. InTech.

Boone, T.B., Aldes, L.D., 1984. Synaptology of the hypoglossal nucleus in the rat. Exp Brain Res 57, 22-32.

Brust, R.D., Corcoran, A.E., Richerson, G.B., Nattie, E., Dymecki, S.M., 2014. Functional and developmental identification of a molecular subtype of brain serotonergic neuron specialized to regulate breathing dynamics. Cell Rep. 9, 2152-2165.

Cameron, W.E., Jodkowski, J.S., Fang, H., Guthrie, R.D., 1991. Electrophysiological properties of developing phrenic motoneurons in the cat. J Neurophysiol 65, 6719.

Cameron, W.E., Nunez-Abades, P.A., 2000. Physiological changes accompanying anatomical remodeling of mammalian motoneurons during postnatal development. Brain Res Bull 53, 523-7.

Carrascal, L., Nieto-Gonzalez, J.L., Cameron, W.E., Torres, B., Nunez-Abades, P.A., 2005. Changes during the postnatal development in physiological and anatomical characteristics of rat motoneurons studied in vitro. Brain Res Brain Res Rev 49, 377-87. https://doi.org/10.1016/j.brainresrev.2005.02.003 
Cooper, M.H., 1981. Neurons of the hypoglossal nucleus of the rat. Otolaryngol Head Neck Surg 89, 10-5. https://doi.org/10.1177/019459988108900103

Cowan, W.M., Fawcett, J.W., O’Leary, D.D., Stanfield, B.B., 1984. Regressive events in neurogenesis. Science 225, 1258-65.

Das, G., Reuhl, K., Zhou, R., 2013. The Golgi-Cox method. Methods Mol Biol 1018, 313-21.

De Carlos, J.A., Borrell, J., 2007. A historical reflection of the contributions of Cajal and Golgi to the foundations of neuroscience. Brain Res. Rev. 55, 8-16. https://doi.org/10.1016/j.brainresrev.2007.03.010

Delignette-Muller, M.L., Dutang, C., 2015. fitdistrplus: An R Package for Fitting Distributions. J. Stat. Softw. Vol 1 Issue 420151.

Donohue, D.E., Ascoli, G.A., 2008. A comparative computer simulation of dendritic morphology. PLoS Comput Biol 4, e1000089. https://doi.org/10.1371/journal.pcbi.1000089

Dotta, A., Mortola, J.P., 1992. Postnatal development of the denervated lung in normoxia, hypoxia, or hyperoxia. J Appl Physiol 1985 73, 1461-6.

Dutta, S., Sengupta, P., 2016. Men and mice: Relating their ages. Life Sci 152, 244-8.

Easter, S.S., Jr., Purves, D., Rakic, P., Spitzer, N.C., 1985. The changing view of neural specificity. Science 230, 507-11.Ekberg, J., Adams, D.J., 2006. Neuronal voltagegated sodium channel subtypes: key roles in inflammatory and neuropathic pain. Int J Biochem Cell Biol 38, 2005-10. https://doi.org/10.1016/j.biocel.2006.06.008

Engel, J., Schultens, H.A., Schild, D., 1999. Small conductance potassium channels cause an activity-dependent spike frequency adaptation and make the transfer function of neurons logarithmic. Biophys J 76, 1310-9. https://doi.org/10.1016/S00063495(99)77293-0

Erickson, J.T., Shafer, G., Rossetti, M.D., Wilson, C.G., Deneris, E.S., 2007. Arrest of 5HT neuron differentiation delays respiratory maturation and impairs neonatal homeostatic responses to environmental challenges. Respir. Physiol. Neurobiol. 159, 85-101. https://doi.org/10.1016/j.resp.2007.06.002

Everitt, B.S., 1996. An introduction to finite mixture distributions. Stat Methods Med Res $5,107-27$.

Feldman, J.L., Del Negro, C.A., 2006. Looking for inspiration: new perspectives on respiratory rhythm. Nat. Rev. Neurosci. 7, 232-242. https://doi.org/10.1038/nrn1871 
Feldman, J.L., Del Negro, C.A., Gray, P.A., 2013. Understanding the rhythm of breathing: so near, yet so far. Annu. Rev. Physiol. 75, 423-452. https://doi.org/10.1146/annurev-physiol-040510-130049

Feldman, J.L., Smith, J.C., 1995. Neural control of respiratory pattern in mammals: an overview, in: Dempsey, J.A., Pack, A.I. (Eds.), Regulation of Breathing. MarcelDekker, New York, N.Y., pp. 39-69.

Ferrante, M., Migliore, M., \& Ascoli, G. A., 2013. Functional impact of dendritic branchpoint morphology. J Neurosci, 33(5), 2156-2165. doi:10.1523/JNEUROSCI.3495-12.2013

Ferreira, T.A., Blackman, A.V., Oyrer, J., Jayabal, S., Chung, A.J., Watt, A.J., Sjostrom, P.J., van Meyel, D.J., 2014. Neuronal morphometry directly from bitmap images. Nat Meth 11, 982-984.

Fietkiewicz, C., Loparo, K.A., Wilson, C.G., 2011. Drive latencies in hypoglossal motoneurons indicate developmental change in the brainstem respiratory network. J. Neural Eng. 8. https://doi.org/10.1088/1741-2560/8/6/065011

Fogarty, M.J., Kanjhan, R., Bellingham, M.C., Noakes, P.G., 2016. Glycinergic Neurotransmission: A Potent Regulator of Embryonic Motor Neuron Dendritic Morphology and Synaptic Plasticity. J Neurosci 36, 80-7.

Forbes, C., Evans, M., Hastings, N., Peacock, B., 2011. Stochastic Modeling, in: Statistical Distributions. Hoboken, New Jersey, pp. 32-43.

Fregosi, R.F., 2011. Respiratory related control of hypoglossal motoneurons--knowing what we do not know. Respir Physiol Neurobiol 179, 43-7. https://doi.org/10.1016/j.resp.2011.06.023

Fregosi, R.F., Pilarski, J.Q., 2008. Prenatal nicotine exposure and development of nicotinic and fast amino acid-mediated neurotransmission in the control of breathing. Respir Physiol Neurobiol 164, 80-6. https://doi.org/10.1016/j.resp.2008.05.008

Fujimoto, S., Yamaguchi, K., Gunjigake, K., 2009. Clinical estimation of mouth breathing. Am J Orthod Dentofac. Orthop 136, 630 e1-7; discussion 630-1. https://doi.org/10.1016/j.ajodo.2009.03.034

Fukunishi, Y., Nagase, Y., Yoshida, A., Moritani, M., Honma, S., Hirose, Y., Shigenaga, Y., 1999. Quantitative analysis of the dendritic architectures of cat hypoglossal motoneurons stained intracellularly with horseradish peroxidase. J Comp Neurol $405,345-58$.

Fulton, B.P., Walton, K., 1986. Electrophysiological properties of neonatal rat motoneurones studied in vitro. J Physiol 370, 651-78. 
García, S., Fernández, A., Luengo, J., Herrera, F., 2010. Advanced nonparametric tests for multiple comparisons in the design of experiments in computational intelligence and data mining: Experimental analysis of power. Inf. Sci. 180, 2044-2064. https://doi.org/10.1016/j.ins.2009.12.010

Ghasemi, A., Zahediasl, S., 2012. Normality tests for statistical analysis: a guide for nonstatisticians. Int J Endocrinol Metab 10, 486-9. https://doi.org/10.5812/ijem.3505

Gibb, R., Kolb, B., 1998. A method for vibratome sectioning of Golgi-Cox stained whole rat brain. J Neurosci Methods 79, 1-4.

Golder, F.J., Zabka, A.G., Bavis, R.W., Baker-Herman, T., Fuller, D.D., Mitchell, G.S., 2005. Differences in time-dependent hypoxic phrenic responses among inbred rat strains. J Appl Physiol 1985 98, 838-44. https://doi.org/10.1152/japplphysiol.00984.2004

González-Castillo, C., Muñoz-Ortiz, E., Guzmán-Brambila, C., Rojas-Mayorquín, A.E., Beltran-Parrazal, L., Ortuño-Sahagún, D., Morgado-Valle, C., 2017. Differential Expression of Ion Channels in Adult and Neonatal Rat Ventral Respiratory Column. J. Mol. Neurosci. https://doi.org/10.1007/s12031-017-1001-2

Guadiana, S.M., Semple-Rowland, S., Daroszewski, D., Madorsky, I., Breunig, J.J., Mykytyn, K., Sarkisian, M.R., 2013. Arborization of dendrites by developing neocortical neurons is dependent on primary cilia and type 3 adenylyl cyclase. $\mathrm{J}$ Neurosci 33, 2626-38.

Hall, R.L., 2005. Energetics of nose and mouth breathing, body size, body composition, and nose volume in young adult males and females. Am J Hum Biol 17, 321-30. https://doi.org/10.1002/ajhb.20122

Harper, R.M., Kinney, H.C., 2010. Potential Mechanisms of Failure in the Sudden Infant Death Syndrome. Curr. Pediatr. Rev. 6, 39-47. https://doi.org/10.2174/157339610791317214

Hartigan, J.A., Hartigan, P.M., 1985. The Dip Test of Unimodality. Ann. Stat. 13, 70-84.

Jan, L.Y., Jan, Y.N., 2012. Voltage-gated potassium channels and the diversity of electrical signalling. J Physiol 590, 2591-9. https://doi.org/10.1113/jphysiol.2011.224212

Johnston, J., Forsythe, I.D., Kopp-Scheinpflug, C., 2010. Going native: voltage-gated potassium channels controlling neuronal excitability. J Physiol 588, 3187-200. https://doi.org/10.1113/jphysiol.2010.191973

Kanjhan, Refik, Fogarty, M.J., Noakes, P.G., Bellingham, M.C., 2016. Developmental changes in the morphology of mouse hypoglossal motor neurons. Brain Struct. Funct. 221, 3755-3786. https://doi.org/10.1007/s00429-015-1130-8 
Kanjhan, R., Noakes, P.G., Bellingham, M.C., 2016. Emerging Roles of Filopodia and Dendritic Spines in Motoneuron Plasticity during Development and Disease. Neural Plast 2016, 3423267. https://doi.org/10.1155/2016/3423267

Khazipov, R., Zaynutdinova, D., Ogievetsky, E., Valeeva, G., Mitrukhina, O., Manent, J.B., Represa, A., 2015. Atlas of the Postnatal Rat Brain in Stereotaxic Coordinates. Front. Neuroanat. 9.

Kinney, H.C., 2009. Brainstem mechanisms underlying the sudden infant death syndrome: evidence from human pathologic studies. Dev Psychobiol 51, 223-33. https://doi.org/10.1002/dev.20367

Kitamura, S., Nishiguchi, T., Sakai, A., 1983. Location of cell somata and the peripheral course of axons of the geniohyoid and thyrohyoid motoneurons: a horseradish peroxidase study in the rat. Exp Neurol 79, 87-96.

Koizumi, H., Wilson, C.G., Wong, S., Yamanishi, T., Koshiya, N., Smith, J.C., 2008. Functional Imaging, Spatial Reconstruction, and Biophysical Analysis of a Respiratory Motor Circuit Isolated In Vitro. J. Neurosci. 28, 2353-2365.

Komendantov, A.O., Ascoli, G.A., 2009. Dendritic excitability and neuronal morphology as determinants of synaptic efficacy. J Neurophysiol 101, 1847-66.

Koyama, Y., 2013. The unending fascination with the Golgi method. OA Anat. 1, 24-31.

Koyama, Y., Nishida, T., Tohyama, M., 2013. Establishment of an optimised protocol for a Golgi-electron microscopy method based on a Golgi-Cox staining procedure with a commercial kit. J Neurosci Methods 218, 103-9. https://doi.org/10.1016/j.jneumeth.2013.05.004

Krichmar, J.L., Nasuto, S.J., Scorcioni, R., Washington, S.D., Ascoli, G.A., 2002. Effects of dendritic morphology on CA3 pyramidal cell electrophysiology: a simulation study. Brain Res 941, 11-28.

Lamanauskas, N., Nistri, A., 2008. Riluzole blocks persistent Na+ and Ca2+ currents and modulates release of glutamate via presynaptic NMDA receptors on neonatal rat hypoglossal motoneurons in vitro. Eur J Neurosci 27, 2501-14.

Lape, R., Nistri, A., 2001. Characteristics of fast $\mathrm{Na}(+)$ current of hypoglossal motoneurons in a rat brainstem slice preparation. Eur J Neurosci 13, 763-72.

Le Boedec, K., 2016. Sensitivity and specificity of normality tests and consequences on reference interval accuracy at small sample size: a computer-simulation study. Vet Clin Pathol 45, 648-656. https://doi.org/10.1111/vcp.12390

Leal, R.B., Gomes, M.C., Granville-Garcia, A.F., Goes, P.S., de Menezes, V.A., 2016. Impact of breathing patterns on the quality of life of 9- to 10-year-old 
schoolchildren. Am J Rhinol Allergy 30, 147-52.

https://doi.org/10.2500/ajra.2016.30.4363

Levine, N.D., Rademacher, D.J., Collier, T.J., O’Malley, J.A., Kells, A.P., San Sebastian, W., Bankiewicz, K.S., Steece-Collier, K., 2013. Advances in thin tissue GolgiCox impregnation: fast, reliable methods for multi-assay analyses in rodent and non-human primate brain. J Neurosci Methods 213, 214-27.

https://doi.org/10.1016/j.jneumeth.2012.12.001

Lewis, P.R., Flumerfelt, B.A., Shute, C.C., 1971. The use of cholinesterase techniques to study topographical localization in the hypoglossal nucleus of the rat. J Anat 110, 203-13.

Lindsey, B.G., Rybak, I.A., Smith, J.C., 2012. Computational models and emergent properties of respiratory neural networks. Compr Physiol 2, 1619-70. https://doi.org/10.1002/cphy.c110016

Liu, Q., Fehring, C., Lowry, T.F., Wong-Riley, M.T., 2009. Postnatal development of metabolic rate during normoxia and acute hypoxia in rats: implication for a sensitive period. J Appl Physiol 1985 106, 1212-22. https://doi.org/10.1152/japplphysiol.90949.2008

Liu, Q., Lowry, T.F., Wong-Riley, M.T.T., 2006. Postnatal changes in ventilation during normoxia and acute hypoxia in the rat: implication for a sensitive period. J Physiol 577, 957-70. https://doi.org/10.1113/jphysiol.2006.121970

Liu, Q., Wong-Riley, M.T.T., 2006. Developmental changes in the expression of GABAA receptor subunits alpha1, alpha2, and alpha3 in brain stem nuclei of rats. Brain Res 1098, 129-38.

Liu, Q., Wong-Riley, M.T., 2003. Postnatal changes in cytochrome oxidase expressions in brain stem nuclei of rats: implications for sensitive periods. J Appl Physiol 1985 95, 2285-91. https://doi.org/10.1152/japplphysiol.00638.2003

Liu, Q., Wong-Riley, M.T.T., 2002. Postnatal expression of neurotransmitters, receptors, and cytochrome oxidase in the rat pre-Botzinger complex. J. Appl. Physiol. 92, 923-934.

Liu, Y.Y., Wong-Riley, M.T., 2001. Developmental study of cytochrome oxidase activity in the brain stem respiratory nuclei of postnatal rats. J Appl Physiol 1985 90, 68594.

Macari, A.T., Bitar, M.A., Ghafari, J.G., 2012. New insights on age-related association between nasopharyngeal airway clearance and facial morphology. Orthod Craniofac Res 15, 188-97. https://doi.org/10.1111/j.1601-6343.2012.01540.x

Marks, W.B., Burke, R.E., 2007a. Simulation of motoneuron morphology in three dimensions. I. Building individual dendritic trees. J Comp Neurol 503, 685-700. 
Marks, W.B., Burke, R.E., 2007b. Simulation of motoneuron morphology in three dimensions. II. Building complete neurons. J Comp Neurol 503, 701-16.

Marsaglia, G., 2006. Ratios of Normal Variables. J. Stat. Softw. 16, 10.

Martin, R.J., Wilson, C.G., 2009. What to do about apnea of prematurity? J Appl Physiol 107, 1015-6. https://doi.org/10.1152/japplphysiol.00940.2009

Martinez-Tellez, R., Gomez-Villalobos Mde, J., Flores, G., 2005. Alteration in dendritic morphology of cortical neurons in rats with diabetes mellitus induced by streptozotocin. Brain Res 1048, 108-15. https://doi.org/10.1016/j.brainres.2005.04.048

McClung, J.R., Goldberg, S.J., 1999. Organization of motoneurons in the dorsal hypoglossal nucleus that innervate the retrusor muscles of the tongue in the rat. Anat Rec 254, 222-30.

Mortola, J.P., 1991. Hamsters versus rats: ventilatory responses in adults and newborns. Respir Physiol 85, 305-17.

Mortola, J.P., 1984. Breathing pattern in newborns. J Appl Physiol Respir Env. Exerc Physiol 56, 1533-40.

Mortola, J.P., Fisher, J.T., 1981. Mouth and nose resistance in newborn kittens and puppies. J Appl Physiol Respir Env. Exerc Physiol 51, 641-5. https://doi.org/10.1152/jappl.1981.51.3.641

Mortola, J.P., Lanthier, C., 2004. Scaling the amplitudes of the circadian pattern of resting oxygen consumption, body temperature and heart rate in mammals. Comp Biochem Physiol Mol Integr Physiol 139, 83-95. https://doi.org/10.1016/j.cbpb.2004.07.007

Myatt, D.R., Hadlington, T., Ascoli, G.A., Nasuto, S.J., 2012. Neuromantic - from semimanual to semi-automatic reconstruction of neuron morphology. Front Neuroinform 6, 4. https://doi.org/10.3389/fninf.2012.00004

Nguyen, Q.T., Wessel, R., Kleinfeld, D., 2004. Developmental regulation of active and passive membrane properties in rat vibrissa motoneurones. J Physiol 556, 203-19. https://doi.org/10.1113/jphysiol.2003.060087

Nunez-Abades, P.A., Cameron, W.E., 1997. Relationship between membrane properties and cell size of developing rat genioglossal motoneurons studied in vitro. Neurosci Lett 223, 41-4.

Nunez-Abades, P.A., Cameron, W.E., 1995. Morphology of developing rat genioglossal motoneurons studied in vitro: relative changes in diameter and surface area of somata and dendrites. J Comp Neurol 353, 129-42. https://doi.org/10.1002/cne.903530112 
Nunez-Abades, P.A., He, F., Barrionuevo, G., Cameron, W.E., 1994. Morphology of developing rat genioglossal motoneurons studied in vitro: changes in length, branching pattern, and spatial distribution of dendrites. J Comp Neurol 339, 40120. https://doi.org/10.1002/cne.903390308

Nunez-Abades, P.A., Spielmann, J.M., Barrionuevo, G., Cameron, W.E., 1993. In vitro electrophysiology of developing genioglossal motoneurons in the rat. $\mathrm{J}$ Neurophysiol 70, 1401-11.

Odutola, A.B., 1976. Cell grouping and Golgi architecture of the hypoglossal nucleus of the rat. Exp Neurol 52, 356-71.

Padzys, G.S., Thornton, S.N., Martrette, J.M., Trabalon, M., 2011. Effects of short term forced oral breathing in rat pups on weight gain, hydration and stress. Physiol Behav 102, 175-80. https://doi.org/10.1016/j.physbeh.2010.10.018

Powell, G.L., Gaddy, J., Xu, F., Fregosi, R.F., Levine, R.B., 2016. Developmental Nicotine Exposure disrupts dendritic arborization patterns of hypoglossal motoneurons in the neonatal rat. Dev Neurobiol. https://doi.org/10.1002/dneu.22379

Powers, R.K., Binder, M.D., 2003. Persistent sodium and calcium currents in rat hypoglossal motoneurons. J Neurophysiol 89, 615-24. https://doi.org/10.1152/jn.00241.2002

Powers, R.K., Sawczuk, A., Musick, J.R., Binder, M.D., 1999. Multiple mechanisms of spike-frequency adaptation in motoneurones. J Physiol Paris 93, 101-14.

Preibisch, S., Saalfeld, S., Tomancak, P., 2009. Globally optimal stitching of tiled 3D microscopic image acquisitions. Bioinformatics 25, 1463-1465.

Purvis, L.K., Butera, R.J., 2005. Ionic current model of a hypoglossal motoneuron. J Neurophysiol 93, 723-33. https://doi.org/10.1152/jn.00703.2004

Ranjan, A., Mallick, B.N., 2012. Differential staining of glia and neurons by modified Golgi-Cox method. J Neurosci Methods 209, 269-79. https://doi.org/10.1016/j.jneumeth.2012.06.023

Rourke, K.S., Mayer, C.A., MacFarlane, P.M., 2016. A critical postnatal period of heightened vulnerability to lipopolysaccharide. Respir Physiol Neurobiol 232, 2634. https://doi.org/10.1016/j.resp.2016.06.003

Sant'Anna, G.M., Mortola, J.P., 2003. Thermal and respiratory control in young rats exposed to cold during postnatal development. Comp Biochem Physiol Mol Integr Physiol 134, 449-59.

Sant’Anna, G.M., Mortola, J.P., 2002. Thermal and respiratory control in young rats with altered caloric intake during postnatal development. Respir Physiol Neurobiol 
133, 215-27.

Sengupta, P., 2013. The Laboratory Rat: Relating Its Age With Human's. Int J Prev Med 4, 624-30.

Sholl, D.A., 1950. A discussion on the measurement of growth and form; the theory of differential growth analysis. Proc R Soc Lond B Biol Sci 137, 470-4.

Smith, J.C., Butera, R.J., Koshiya, N., Negro, C.D., Wilson, C.G., Johnson, S.M., 2000. Respiratory rhythm generation in neonatal and adult mammals: the hybrid pacemaker-network model. Respir. Physiol. 122, 131-147.

Smith, J.C., Ellenberger, H.H., Ballanyi, K., Richter, D.W., Feldman, J.L., 1991. PreBötzinger complex: a brainstem region that may generate respiratory rhythm in mammals. Science 254, 726-729.

Standard Practice for Characterization of Particles (No. F 1877-98), 2003. ASTM.

Strohl, K.P., Thomas, A.J., St Jean, P., Schlenker, E.H., Koletsky, R.J., Schork, N.J., 1997. Ventilation and metabolism among rat strains. J Appl Physiol 1985 82, 317-23. https://doi.org/10.1152/jappl.1997.82.1.317

Takasu, N., Hashimoto, P.H., 1988. Morphological identification of an interneuron in the hypoglossal nucleus of the rat: a combined Golgi-electron microscopic study. J Comp Neurol 271, 461-71. https://doi.org/10.1002/cne.902710313

Thoby-Brisson, M., Ramirez, J.-M., 2001. Identification of Two Types of Inspiratory Pacemaker Neurons in the Isolated Respiratory Neural Network of Mice. J. Neurophysiol. 86, 104-112.

Thoby-Brisson, M., Ramirez, J.-M., 2000. Role of Inspiratory Pacemaker Neurons in Mediating the Hypoxic Response of the Respiratory Network lemphIn Vitro. J. Neurosci. 20, 5858-5866.

Vacher, H., Mohapatra, D.P., Trimmer, J.S., 2008. Localization and targeting of voltagedependent ion channels in mammalian central neurons. Physiol Rev 88, 1407-47. https://doi.org/10.1152/physrev.00002.2008

Wong-Riley, M.T., Liu, Q., 2005. Neurochemical development of brain stem nuclei involved in the control of respiration. Respir Physiol Neurobiol 149, 83-98. https://doi.org/10.1016/j.resp.2005.01.011

Wong-Riley, M.T., Liu, Q., Gao, X.P., 2013. Peripheral-central chemoreceptor interaction and the significance of a critical period in the development of respiratory control. Respir Physiol Neurobiol 185, 156-69.

https://doi.org/10.1016/j.resp.2012.05.026 
Yang, X.X., Powell, W.S., Xu, L.J., Martin, J.G., 1999. Strain dependence of the airway response to dry-gas hyperpnea challenge in the rat. J Appl Physiol 1985 86, $152-$ 8. https://doi.org/10.1152/jappl.1999.86.1.152

Yoshimoto, R., Iwasaki, S., Takago, H., Nakajima, T., Sahara, Y., Kitamura, K., 2015. Developmental increase in hyperpolarization-activated current regulates intrinsic firing properties in rat vestibular ganglion cells. Neuroscience 284, 632-42. https://doi.org/10.1016/j.neuroscience.2014.10.034

Yu, F.H., Catterall, W.A., 2003. Overview of the voltage-gated sodium channel family. Genome Biol 4, 207.

Zubler, F., Douglas, R., 2009. A Framework for Modeling the Growth and Development of Neurons and Networks. Front. Comput. Neurosci. 3, 25. 